\title{
On positive linear operators
}

by

Farzaneh Jannat

A thesis submitted to the Faculty of Graduate Studies of
The University of Manitoba
in partial fulfilment of the requirements of the degree of

MASTER OF SCIENCE

Department of Mathematics

University of Manitoba

Winnipeg

Copyright (c) 2019 by Farzaneh Jannat 


\begin{abstract}
Approximation theory received much attention in the last century, and an intriguing area in this field is positive linear operators. This thesis is a literature survey on positive linear operators. We discuss some of the well-known examples of positive linear operators. Preserving $k$-monotonicity by positive linear operators is another interesting topic in this area. We are interested if $L_{n}(f ; x)$ is $k$-monotone whenever function $f$ is $k$-monotone. For a sequence of positive linear operators $\left\{L_{n}(f ; x)\right\}$, it is a natural question if this sequence converges to $f(x)$ and how fast is this convergence. We study the saturation of these operators. Usually, there is a relation between the rate of convergence and the smoothness of the function being approximated. If increasing smoothness of functions does not result in the increase of the degree of approximation, this phenomenon is called saturation. We discuss iteration of general positive linear operators and give numerical examples.
\end{abstract}




\section{Acknowledgements}

I would like to express my gratitude to my advisor Professor Kopotun for the continuous support of my M.Sc. thesis, for his patience and immense knowledge. His guidance helped me in all the time of writing of this thesis.

In addition, I would like to thank my thesis committee members: Professor Prymak and Professor Leblanc for their insightful comments. I would also thank the Department of Mathematics for the financial support throughout my studies. 


\section{Contents}

$\begin{array}{lll}1 & \text { Introduction } & 1\end{array}$

1.1 Applications of positive linear operators . . . . . . . . . . . . . . 4

\begin{tabular}{lll}
\hline 2 & Preliminaries & 7
\end{tabular}

2.1 Notations . . . . . . . . . . . . . . . . . . . . 7

2.2 Definitions . . . . . . . . . . . . . . . . . . . . . . . 10

2.2 .1 Basics . . . . . . . . . . . . . . . . . 10

2.2 .2 Positive Linear Operator . . . . . . . . . . . . . . . . . . . 13

2.2 .3 Moduli of Smoothness . . . . . . . . . . . . . . . . . 15

3 Some Examples of Positive Linear Operators 16

3.1 Bernstein operator $\ldots \ldots \ldots \ldots$

3.2 de la Vallée Poussin operator . . . . . . . . . . . . . . . . . . . . . . . 18

3.3 Fejér operator and Jackson operator . . . . . . . . . . . . . . . . . . . 19

3.4 Kantorovich operator . . . . . . . . . . . . . . . . . . . . 22

3.5 Bernstein-Chlodovsky operator . . . . . . . . . . . . . 23

3.6 Favard-Szász-Mirakjan operator . . . . . . . . . . . . . . . . . . . . . 24

3.7 Baskakov operator $\ldots \ldots \ldots \ldots \ldots$

3.8 Durrmeyer operator.$\ldots \ldots \ldots \ldots$

3.9 Bleimann-Butzer-Hahn operator . . . . . . . . . . . . . . . . . . 28

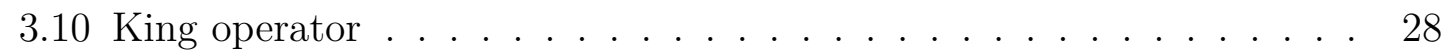


$4 \quad K$-monotonicity Preservation by Positive Linear Operators 31

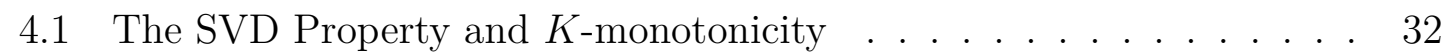

4.2 The Relation Between Total Positivity and $K$-monotonicity . . . . . . 35

4.3 Approximation of Convex Functions by Positive Linear Operators . . 40

5 Convergence Theory of Positive Linear Operators 47

5.1 The Bohman-Korovkin Theorem . . . . . . . . . . . . . . . . . . . . 47

5.2 A Korovkin-type Theorem for Unbounded Functions with Non-compact Support . . . . . . . . . . . . . . . . . . 55

5.3 Shisha-Mond Quantitative Estimates . . . . . . . . . . . . . . . . 58

5.4 Voronovskaja's Formula . . . . . . . . . . . . . . . . . . . . 60

$\begin{array}{lll}6 & \text { Saturation of Positive Linear Operators } & 71\end{array}$

$6.1 \quad$ Saturation in $C_{2 \pi} \ldots \ldots \ldots \ldots$. . . . . . . . . . . . . . . 71

6.2 Inverse and Direct Theorems . . . . . . . . . . . . . . . . . . . . . . . 72

6.2.1 The Tureckiř Theorem and its extension . . . . . . . . . . . . 73

6.2 .2 Sunouchi-Watari Theorem . . . . . . . . . . . . . . . . . 75

6.3 Saturation in $C[a, b] \quad \ldots \ldots \ldots \ldots$. . . . . . . . . . . 77

$\begin{array}{|ll|}7 & \text { The Iteration of Positive Linear Operators }\end{array}$

\begin{tabular}{lr}
\hline Bibliography & 90
\end{tabular}

\begin{tabular}{lr}
\hline Index & 97
\end{tabular} 


\section{List of Figures}

$3.1 \quad$ Approximation of $f(x)=1+\cos (7 \pi x)$ by Bernstein polynomials $B_{n}$ with $n=10,50,100 . \quad \ldots \ldots \ldots \ldots \ldots \ldots$

$3.2 \quad$ Approximation of $h(x)$ by Fejér operators for $n=10 . \quad \ldots \ldots$. . 20

$3.3 \quad$ Approximation of $h(x)$ by Fejér operators for $n=100 . \ldots \ldots$

3.4 Kernels of convolution operators: Fejér, Jackson and de la Vallée Poussin. . . . . . . . . . . . . . . . . . . . . 22

3.5 Approximation of $f(x)=2+e^{-x}+\frac{3 x^{2}}{\left(x^{2}+1\right)^{2}}$ by Bernstein and FavardSzász-Mirakjan operators for $n=8 . \ldots \ldots \ldots \ldots \ldots$

$3.6 \quad$ Approximation of $f(x)$ from (3.8) by Bernstein and Durrmeyer oper-

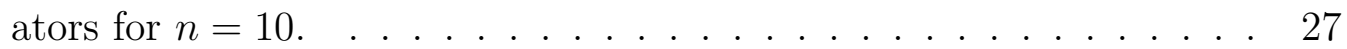

$3.7 \quad$ Approximation of $h(x)=\frac{(x+1)(x+5)}{x^{4}+100}$ by $\mathcal{B B H}$ operator for $n=5$, $n=20$, and $n=80$. . . . . . . . . . . . . . . . . . 29

$3.8 \quad$ Approximation of $f(x)=2+\left(x^{2}+1\right)^{2}$ by Bernstein and King operators for $n=3 . \ldots \ldots \ldots \ldots \ldots \ldots \ldots$

$4.1 \quad$ Approximation of $f(x)=2+x^{2} . \ldots \ldots \ldots \ldots \ldots \ldots$

4.2 Approximation of function $f(x)=4+x^{4}$ by the Bernstein operator and modified Durrmeyer operator. . . . . . . . . . . . . . . . . 44

$4.3 \quad$ Approximation of function $f(x)=4+x^{4}$ by Bernstein operator and Durrmeyer operator for $n=4$.

7.1 Iterates of the Bernstein operator of order $n=5 . \ldots \ldots$. . . 81 
$7.2 \quad$ Iterates of the King operator for $f(x)=2+x^{4}$ when $n=5$. . . . . . 87 
1

\section{Introduction}

All exact science is dominated by the idea of approximation.

Bertrand Russell

Positive linear operators is a simple but one of the powerful concepts used in approximation theory. For example, the Weierstrass theorem can be proved using positive linear operators (Bernstein operator s). This theorem states that if $f$ is a continuous function on $[0,1]$, then for any given $\epsilon>0$, there exists a polynomial $P(x)$ such that

$$
|f(x)-P(x)|<\epsilon, \quad \text { for } \quad 0 \leq x \leq 1 .
$$

In 1912, Bernstein gave a constructive proof of this theorem. He introduced the polynomial operator $B_{n}(f ; x)$ defined by

$$
B_{n}(f ; x):=\sum_{k=0}^{n}\left(\begin{array}{l}
n \\
k
\end{array}\right) x^{k}(1-x)^{n-k} f\left(\frac{k}{n}\right),
$$

and showed that $\left|f(x)-B_{n}(f ; x)\right|<\epsilon, 0 \leq x \leq 1$, for sufficiently large $n$ (depending on $\epsilon)$.

The Bernstein operator has many useful properties and has been the object of intense interest to mathematicians. However, there are certain drawbacks associated 
with this operator one of them being its slow convergence. Over the years, a lot of research was done to generalize this operator and study properties of "Bernstein-like" operators.

In 1953, Pavel Korovkin [36] (Doklady Akad. Nauk SSSR) introduced the notion of "positive linear operator" in approximation theory, and a related paper was already published by Harald Bohman [10] (Arkiv för Matematik) in 1952. Their seminal result is now known as the Bohman-Korovkin Theorem (see Theorem 5.1 later in this thesis). We also mention that some related work in this area was done by Tiberiu Popoviciu in 1951 (see [50, p. 10]). The history in this area in 1950s is well described in Frans Schurer's Ph.D. thesis [50] "On linear positive operators in approximation theory" defended in 1965, where the Bohamn-Korovkin theorem was extended.

This thesis is a survey on positive linear operators and is composed of seven chapters. Among other things, we discuss convergence, saturation and preservation of $k$-monotonicity by these operators.

Chapter 3 is dedicated to some of the well-known examples of positive linear operators. They are sorted chronologically from the Bernstein operator (1912) to the King operator (2003). We include several figures to illustrate the relationship among these operators and their approximation orders. We also introduce and discuss interpolation and convolution operators in this chapter.

We explicitly calculate $L_{n}\left(t^{i} ; x\right)$ for $i=0,1,2$, since, as we show in Chapter 5 . this is critical for showing the convergence of these operators.

At the end of this chapter, in Section 4.3, after explaining the meaning of "better approximation" of a convex function, it is discussed which of these operators (and under what conditions) better approximate convex functions. Moreover, it is shown that among all positive polynomial operators on $C[0,1]$ that preserve affine functions 
and have the form

$$
L(f ; x)=\sum_{k=0}^{n}\left(\begin{array}{l}
n \\
k
\end{array}\right) x^{k}(1-x)^{n-k} \int_{0}^{1} f d \mu_{k, n},
$$

where $\mu_{k, n}$ is a positive Borel measure on $[0,1]$, Bernstein operator provides the "best approximation" of convex functions.

The aim of Chapter 4 is to discuss preservation of $k$-monotonicity by positive linear operators. Various approaches have been proposed to find conditions that guarantee that an operator preserves $k$-monotonicity. A preliminary work in this field focused on finding the $k$ th derivative of the operator in terms of divided differences. For instance, the $k$ th derivative of the Bernstein operator and the Bleimann-ButzerHahn operator [1, Theorem 6.1] can be written in this way. However, this method cannot easily be applied for many operators. In section 4.1, it is shown that there is a remarkable relation between the strong variation diminishing property (SVD) and $k$-monotonicity.

Chapter 5 is devoted to discussing convergence of positive linear operators. Let $f$ be a bounded and continuous function. The Bohman-Korovkin Theorem guarantees the uniform convergence of a sequences of linear positive operators $\left\{L_{n}(f ; x)\right\}$ to $f$ if $\left\{L_{n}\left(t^{m} ; x\right)\right\}$ converges to $x^{m}$ for $m=0,1,2$. Note that this theorem does not work for unbounded functions on non-compact domains. As is illustrated in section 5.2 there is a positive linear operator such that $\left\{L_{n}\left(t^{m} ; x\right)\right\}$ converges to $x^{m}$ for $m=0,1,2$ but $\left\{L_{n}(f ; x)\right\}$ does not converge to $f$. We discuss the approach of Boyanov and Veselinov, [12, Theorem 2] that can be used to overcome this obstacle. The second part of Chapter 5 discusses the Voronovskaja theorem. It turns out that it is important to know

$$
\lim _{n \rightarrow \infty} n\left(L_{n}(f ; x)-f(x)\right)
$$

in order to obtain the information about the rate of convergence and saturation 
properties of the sequence $\left\{L_{n}\right\}$ of positive linear operators. In 1932, Voronovskaja calculated this limit for the Bernstein operator $B_{n}(f)$ and showed that

$$
\lim _{n \rightarrow \infty} n\left(B_{n}(f ; x)-f(x)\right)=\frac{x(1-x)}{2} f^{\prime \prime}(x) .
$$

Hence, if the second derivative of a function $f$ is nonzero, then the rate of convergence of $B_{n}(f)$ to $f$ is not better than $1 / n$.

Chapter 6 is devoted to saturation of positive linear operators. The concept of saturation was introduced by Favard in 1947. The saturation class for a positive linear operator is the class of all functions in the domain of the operator which have the best possible degree of approximation by this operator. After a brief history and appropriate definitions, we discuss the Tureckir and Sunouchi-Watari theorems. In order to illustrate these theorems, we calculate saturation orders for Fejér, Jackson and Bernstein operators.

Finally, the concept of iteration of positive linear operators (that was introduced by Kelisky and Rivlin in 1967 for the Bernstein operator) is the main topic of Chapter 7. The application of iterations of operators is mentioned such as increasing the order of approximation.

\subsection{Applications of positive linear operators}

Positive linear operators have numerous applications in such areas as Differential Equations, Computer Aided Geometric Design (CAGD), Mathematical Finance, Statistics, etc. In this section, we give two examples of how they can be used to solve (or approximate solutions of) certain differential equations. 
Consider the initial value problem (see [15, p. 249])

$$
\left\{\begin{array}{l}
y^{\prime}=f(x, y) \\
y(0)=y_{0}
\end{array}\right.
$$

where $f$ and its first two partial derivatives are bounded in the strip $0 \leq x<1$, $-\infty<y<\infty$, and $f$ satisfies the Lipschitz condition with respect to the second variable, i.e.,

$$
\left|f\left(x, y_{1}\right)-f\left(x, y_{2}\right)\right| \leq \lambda\left|y_{1}-y_{2}\right|, \quad \text { with } 0 \leq \lambda<1
$$

Let the sequence $\left\{z_{n}\right\}$ be defined as $z_{0}(x)=y_{0}$ and for $n \geq 1$

$$
z_{n}(x)=y_{0}+\int_{0}^{x} M_{n}\left(f\left(t, y_{n-1}(t)\right) ; s\right) d s
$$

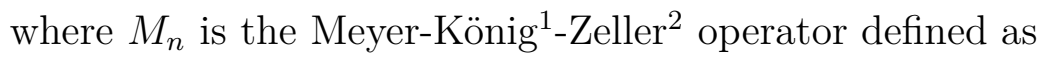

$$
M_{n}(f ; x):=(1-x)^{n+1} \sum_{k=0}^{\infty}\left(\begin{array}{c}
n+k \\
k
\end{array}\right) x^{k} f\left(\frac{k}{n+k}\right), \quad \text { for } \quad x \in[0,1) \text {, }
$$

and $M_{n}(f ; 1)=f(1)$. Cheney and Sharma [15, p. 249] showed that the sequence $\left\{z_{n}\right\}$ converges uniformly to the solution of 1.1 .

Another application of these operators in Differential Equations is the solution of Dirichlet's Problem on the unit disc. Let $C_{2 \pi}$ denotes the set of all continuous $2 \pi$

\footnotetext{
${ }^{1}$ Werner Meyer-König (1912-2002) was a German mathematician who worked in analysis and approximation theory. From 1930, he studied mathematics and physics at the University of Tübingen, the University of Kiel and the University of Stuttgart. He became an assistant in Stuttgart and was awarded his doctorate in 1939 by K. Knopp in Tübingen.https://de.wikipedia.org/ wiki/Werner_Meyer

${ }^{2}$ Karl Longin Zeller (1924-2006) was a German mathematician and computer scientist who worked in numerical analysis and approximation theory. He earned his Ph.D. from the University of Tübingen in 1950, under the supervision of K. Knopp and E. Kamke. The title of his thesis was "General properties of matrix transformations." He was one of the founders of computer science in Germany. https://en.wikipedia.org/wiki/Karl_Longin_Zeller
} 
periodic functions with

$$
\|f\|_{C_{2 \pi}}:=\sup _{|x| \leq \pi}|f(x)| .
$$

The Abel-Poisson operator is defined as

$$
P_{r}(f ; x):=\frac{1}{2 \pi} \int_{-\pi}^{\pi} f(x-\tau) p_{r}(\tau) d \tau,
$$

where $p_{r}(\tau)$ is the Abel-Poisson kernel

$$
p_{r}(\tau):=1+2 \sum_{k=1}^{\infty} r^{k} \cos k \tau=\frac{1-r^{2}}{1-2 r \cos \tau+r^{2}} .
$$

Dirichlet's problem on the unit disc is that of finding a periodic function $u(x, r)$ in $x$ defined for all $x$ and $r \in(0,1)$ such that the following conditions are satisfied:

1. $\frac{\partial^{2} u(x, r)}{\partial r^{2}}$ and $\frac{\partial^{2} u(x, r)}{\partial x^{2}}$ exist for all $x$ and $r \in(0,1)$,
2. $\frac{\partial^{2} u(x, r)}{\partial r^{2}}+\frac{1}{r} \frac{\partial u(x, r)}{\partial r}+\frac{1}{r^{2}} \frac{\partial^{2} u(x, r)}{\partial x^{2}}=0$ for $x \in[-\pi, \pi]$ and $r \in(0,1)$,

3. $\|u(\cdot ; r)\|_{C_{2 \pi}} \leq M$ uniformly for $r \in(0,1)$ and

$$
\lim _{r \rightarrow 1^{-}}\|u(\cdot ; r)-f(\cdot)\|_{C_{2 \pi}}=0 .
$$

The next theorem shows that the Abel-Poisson operator is the solution for Dirichlet's problem.

Theorem 1.1 ([13, Theorem 7.1.3, p. 286]). The unique solution of Dirichlet's problem on the unit disc is given by the singular integral of Abel-Poisson. Thus, for all $x \in \mathbb{R}$ and $r \in[0,1)$ we have,

$$
u(x, r)=P_{r}(f ; x)=\frac{1}{2 \pi} \int_{-\pi}^{\pi} f(x-\tau) \frac{1-r^{2}}{1-2 r \cos \tau+r^{2}} d \tau .
$$


2

\section{Preliminaries}

\subsection{Notations}

\begin{tabular}{|l|l|}
\hline$\left[x_{0}, x_{1}, \ldots, x_{k} ; f\right]$ & the divided difference of $f$ on the points $x_{0}, x_{1}, \ldots, x_{k}$ \\
\hline$\Delta_{h}^{k} f(t)$ & $k$ th forward difference \\
\hline$\|f\|_{C_{2 \pi}}$ & the norm on $C_{2 \pi}$ defined by $\|f\|_{C_{2 \pi}}=\sup _{|x| \leq \pi}|f(x)|$ \\
\hline$\|f\|_{*}$ & the norm on $C_{2}^{*}[0, \infty)$ defined by $\|f\|_{*}=\sup _{x \geq 0} \frac{|f(x)|}{1+x^{2}}$ \\
\hline$B_{n}(f ; x)$ & Bernstein operator \\
\hline$C(S)$ & the space of all continuous real-valued functions defined on the \\
& set $S$ \\
\hline$C^{q}(S)$ & the space of all $q$ times continuously differentiable real-valued \\
functions on $S$ \\
\hline$C_{2 \pi}$
\end{tabular}




\begin{tabular}{|c|c|}
\hline$C_{2}^{*}[0, \infty)$ & $\begin{array}{l}\text { the weighted space of all continuous real-valued functions } \\
\text { on }[0, \infty) \text { such that } \lim _{x \rightarrow \infty} \frac{f(x)}{1+x^{2}} \text { exists }\end{array}$ \\
\hline$C_{n}(f ; x)$ & Bernstein-Chlodovsky operator \\
\hline $\mathcal{D}_{n}(f ; x)$ & Bernstein-Durrmeyer operator \\
\hline$e_{i}(x)$ & Korovkin test-function, i.e., $e_{i}(x):=x^{i}, i \geq 0$ \\
\hline $\mathcal{F}[a, b]$ & the space of all real-valued functions on $[a, b]$ \\
\hline $\mathcal{F}_{n}(f ; x)$ & Favard-Szász and Mirakjan operator \\
\hline$f^{(r)}$ & the $r$ th derivative of the function $f$ \\
\hline$\tilde{f}$ & the conjugate of function $f \in C^{*}$ \\
\hline $\mathcal{J}_{n}(f ; x)$ & Jackson operator \\
\hline Lip 1 & the class of all functions $f$ such that $\omega(f, t) \leq M t$ \\
\hline$L_{2 \pi}^{p}(\mathbb{R})$ & $\begin{array}{l}\text { For } 1 \leq p<\infty, L_{2 \pi}^{p}(\mathbb{R}) \text { denotes the Banach space of all } \\
\text { functions } f: \mathbb{R} \rightarrow \mathbb{R} \text { which are Lebesgue integrable to the } p \text {-th } \\
\text { power over }[-\pi, \pi] \text { and } 2 \pi \text { periodic, i.e., } f(x+2 \pi)=f(x) \text { a.e } \\
\text { for } x \in \mathbb{R} \text {. }\end{array}$ \\
\hline$L_{p}^{r}[0,1]$ & $\begin{array}{l}\text { the class of all functions } f \text { with } f^{(r-1)} \text { absolutely continuous } \\
\text { on }[0,1] \text {, and } f^{(r)} \in L_{p}[0,1] \text {. Here, } p \geq 1 \text { and } r \in \mathbb{N} \text {. }\end{array}$ \\
\hline $\operatorname{Lip}_{M}^{*} 2$ & $\begin{array}{l}\text { the space of all functions in } C_{2 \pi} \text { such that for } x, t \in[-\pi, \pi] \\
|f(x+t)-2 f(x)+f(x-t)| \leq 2 M t^{2}\end{array}$ \\
\hline$M_{n}(f ; x)$ & Meyer-König and Zeller operator \\
\hline$n^{\bar{k}}$ & is equal to $n(n+1) \ldots(n+k-1)$ \\
\hline $\mathbb{N}$ & $\{1,2,3, \ldots\}$, the set of natural numbers \\
\hline
\end{tabular}




\begin{tabular}{|l|l|}
\hline $\mathbb{N}_{0}$ & $\mathbb{N} \cup\{0\}$ \\
\hline$P_{r}(f ; x)$ & Abel-Poisson operator \\
\hline $\mathbb{R}$ & the set of real numbers \\
\hline$V_{n}(f ; x)$ & King operator \\
\hline$\nu_{[0,1]}(f)$ & the number of changes of sign of function $f$ in $[0,1]$ \\
\hline$\rho_{k, n}$ & the coefficient of the Fourier series \\
\hline$\sigma_{n}(f ; x)$ & Fejér operator \\
\hline$\omega_{k}(t)$ & modulus of smoothness of order $k$ \\
\hline
\end{tabular}




\subsection{Definitions}

In this section, we will review some basic definitions related to positive linear operators.

This section has three subsections. First, we recall several useful definitions such as those of divided differences, $k$-monotonicity, Chebyshev system, Descartes system, etc. In the second subsection, the definition of a positive linear operator is given. We briefly discuss several different kinds of operators such as a polynomial operator and centered Bernstein-type operator. Finally, we define the moduli of continuity/smoothness.

\subsubsection{Basics}

Definition 2.1 (Big-O $\mathcal{O}$ and small-o notation). We say that $f(n)=\mathcal{O}(g(n))$ if there exists $n_{0} \in \mathbb{N}$ and $C>0$ such that

$$
|f(n)| \leq C|g(n)|, \quad \text { for all } n>n_{0}
$$

and we say that $f(n)=o(g(n))$ if

$$
\lim _{n \rightarrow \infty} \frac{f(n)}{g(n)}=0
$$

Definition 2.2 ( $k$ th forward differences and divided difference, see e.g. [22, p. 121]). For a real valued function $f$, let

$$
\Delta_{h}^{k} f(t)=\sum_{j=0}^{k}(-1)^{k-j}\left(\begin{array}{l}
k \\
j
\end{array}\right) f(t+j h)
$$

be the $k$ th forward difference, and the divided difference of $f$ on the points $x_{0}, x_{1}, \ldots, x_{k}$ 
is denoted by

$$
\left[x_{0}, x_{1}, \ldots, x_{k} ; f\right]:=\sum_{i=0}^{k} \frac{f\left(x_{i}\right)}{l^{\prime}\left(x_{i}\right)},
$$

where $l(x)=\left(x-x_{0}\right) \ldots\left(x-x_{k}\right)$, where $l^{\prime}(x)$ is derivative of $l(x)$.

Definition 2.3 (k-monotonicity). Let $k \geq 1$. A function $f:[a, b] \rightarrow \mathbb{R}$ is said to be $k$-monotone on $[a, b]$ if, for all choices of $(k+1)$ distinct points $x_{0}, x_{1}, \ldots, x_{k}$ in $[a, b]$, the inequality

$$
\left[x_{0}, x_{1}, \ldots, x_{k} ; f\right] \geq 0
$$

holds.

Definition 2.4 (Chebyshev system, see e.g. [37, p.42]). A system of functions $\left\{f_{i}(x)\right\}_{i=0}^{n}$ continuous in the interval $[a, b]$ is called a Chebyshev system if any polynomial

$$
F(x)=\alpha_{0} f_{0}(x)+\alpha_{1} f_{1}(x)+\cdots+\alpha_{n} f_{n}(x), \quad \alpha_{0}^{2}+\cdots+\alpha_{n}^{2} \neq 0
$$

has not more than $n$ zeros in this interval.

Proposition 2.5. The following conditions are equivalent.

1. For any $n+1$ distinct points $\left\{t_{0}, t_{1}, \ldots, t_{n}\right\}$ in $[a, b]$, the following determinant is not zero:

$$
D\left(f_{0}, f_{1}, \ldots, f_{n} ; t_{0}, t_{1}, \ldots, t_{n}\right)=\left|\begin{array}{cccc}
f_{0}\left(t_{0}\right) & f_{0}\left(t_{1}\right) & \ldots & f_{0}\left(t_{n}\right) \\
f_{1}\left(t_{0}\right) & f_{1}\left(t_{1}\right) & \ldots & f_{1}\left(t_{n}\right) \\
\vdots & \vdots & \vdots & \vdots \\
f_{n}\left(t_{0}\right) & f_{n}\left(t_{1}\right) & \ldots & f_{n}\left(t_{n}\right)
\end{array}\right|
$$

2. $\left\{f_{0}(x), f_{1}(x), \ldots, f_{n}(x)\right\}$ is a Chebyshev system. 
The proof is straightforward. Let $\left\{f_{i}(x)\right\}_{i=0}^{n}$ be a Chebyshev system and $\left\{t_{0}, t_{1}, \ldots, t_{n}\right\}$ be an arbitrary collection of $n+1$ distinct points in $[a, b]$. Based on the definition, the system of equations

$$
\alpha_{0} f_{0}\left(t_{i}\right)+\alpha_{1} f_{1}\left(t_{i}\right)+\cdots+\alpha_{n} f_{n}\left(t_{i}\right)=0
$$

with $n+1$ equations and $n+1$ unknowns $\alpha_{i}$ for $i=0,1, \ldots, n$ has only the trivial solution $\alpha_{0}=\alpha_{1}=\cdots=\alpha_{n}=0$. Hence, $D\left(f_{0}, f_{1}, \ldots, f_{n} ; t_{0}, t_{1}, \ldots, t_{n}\right) \neq 0$. Conversely, if this determinant is not zero, then the system (2.1) has only the trivial solution, and so $\left\{f_{i}(x)\right\}_{i=0}^{n}$ is a Chebyshev system.

As an example, $\left\{\varphi_{0}, \varphi_{1}, \varphi_{2}\right\}=\left\{1, \frac{t}{t+1},\left(\frac{t}{t+1}\right)^{2}\right\}$ is a Chebyshev system on $[0, b]$. Indeed, for any three distinct points $t_{0}, t_{1}, t_{2}$, we have

$$
\begin{aligned}
D\left(\varphi_{0}, \varphi_{1}, \varphi_{2} ; t_{0}, t_{1}, t_{2}\right) & =\left|\begin{array}{ccc}
1 & 1 & 1 \\
\frac{t_{0}}{t_{0}+1} & \frac{t_{1}}{t_{1}+1} & \frac{t_{2}}{t_{2}+1} \\
\left(\frac{t_{0}}{t_{0}+1}\right)^{2}\left(\frac{t_{1}}{t_{1}+1}\right)^{2} & \left(\frac{t_{2}}{t_{2}+1}\right)^{2}
\end{array}\right| \\
& =\frac{1}{\left(1+t_{0}\right)^{2}\left(1+t_{1}\right)^{2}\left(1+t_{2}\right)^{2}}\left|\begin{array}{ccc}
1 & 1 & 1 \\
t_{0} & t_{1} & t_{2} \\
t_{0}^{2} & t_{1}^{2} & t_{2}^{2}
\end{array}\right| \\
& =\frac{\left(t_{1}-t_{0}\right)\left(t_{2}-t_{0}\right)\left(t_{2}-t_{1}\right)}{\left(1+t_{0}\right)^{2}\left(1+t_{1}\right)^{2}\left(1+t_{2}\right)^{2}} \neq 0,
\end{aligned}
$$

and so $\left\{\varphi_{0}(t), \varphi_{1}(t), \varphi_{2}(t)\right\}$ is a Chebyshev system.

Definition 2.6 (Descartes system, see e.g. [32, p. 25]). Let $\left\{\varphi_{1}(x), \varphi_{2}(x), \ldots, \varphi_{n}(x)\right\}$ be a Chebyshev system and, for $a<x_{1}<x_{2}<\cdots<x_{p}<b$ and every $1 \leq i_{1}<i_{2}<$ 
$\cdots<i_{p} \leq n$

$$
\Phi\left(\begin{array}{ccc}
x_{1} & \ldots & x_{p} \\
i_{1} & \ldots & i_{p}
\end{array}\right):=\left|\begin{array}{ccc}
\varphi_{i_{1}}\left(x_{1}\right) & \ldots & \varphi_{i_{p}}\left(x_{1}\right) \\
\vdots & \vdots & \vdots \\
\varphi_{i_{1}}\left(x_{p}\right) & \ldots & \varphi_{i_{p}}\left(x_{p}\right)
\end{array}\right|>0
$$

Then, the system $\left\{\varphi_{1}(x), \varphi_{2}(x), \ldots, \varphi_{n}(x)\right\}$ is called a Descartes system.

By definition, Descartes system is Chebyshev system but the reverse is not necessarily correct. For example, $\{-1,-x\}$ is a Chebyshev system on any interval but it is not a Descartes system.

\subsubsection{Positive Linear Operator}

Definition 2.7 ([37, pp. 10-11]). An operator $L(f ; x)$ is said to be linear if the domain of its existence together with the functions $f(t)$ and $g(t)$ contains the functions $\alpha f+\beta g$ and if there holds the equality

$$
L(\alpha f+\beta g ; x)=\alpha L(f ; x)+\beta L(g ; x), \quad \text { for all } \alpha, \beta \in \mathbb{R} .
$$

A linear operator $L(f ; x)$ is said to be positive on the set $E$ if $L(f ; x) \geq 0, x \in E$, holds for every positive function $f$.

Note that, throughout this thesis, we say that a function $f$ is positive on a set $E$ if $f(x) \geq 0$, for all $x \in E$.

For instance, it is easy to see that if an operator is defined as

$$
L(f ; x):=\sum_{k=0}^{n} f\left(x_{k}\right) \varphi_{k}(x),
$$


where $\varphi_{k}(x), 0 \leq k \leq n$, are some fixed functions, then

$$
\begin{aligned}
L(\alpha f+\beta g ; x) & =\sum_{k=0}^{n}\left(\alpha f\left(x_{k}\right)+\beta g\left(x_{k}\right)\right) \varphi_{k}(x) \\
& =\alpha \sum_{k=0}^{n} f\left(x_{k}\right) \varphi_{k}(x)+\beta \sum_{k=0}^{n} g\left(x_{k}\right) \varphi_{k}(x) \\
& =\alpha L(f ; x)+\beta L(g ; x)
\end{aligned}
$$

Therefore, $L$ is a linear operator. Also, this operator $L(f ; x)$ is positive if, for all $k$, $\varphi_{k}(x)$ are positive. Operators of type (2.2) are usually called interpolation operators. Note, however, that these operators $L(f ; x)$ do not actually interpolate $f$ at $\left\{x_{k}\right\}$, i.e.,

$$
L\left(f ; x_{k}\right) \neq f\left(x_{k}\right)
$$

Let $K(t, x)$ be a continuous function with respect to $t \in[a, b]$ (for all $x$ ). Then, the operator

$$
L(f ; x):=\int_{a}^{b} K(t, x) f(t) d t
$$

is positive if $K(t, x)$ is positive and linear since

$$
\begin{aligned}
L(\alpha f+\beta g ; x) & =\int_{a}^{b} K(t, x)(\alpha f(t)+\beta g(t)) d t \\
& =\alpha \int_{a}^{b} K(t, x) f(t) d t+\beta \int_{a}^{b} K(t, x) g(t) d t \\
& =\alpha L(f ; x)+\beta L(g ; x) .
\end{aligned}
$$

Throughout the thesis, we use the term $\left\{L_{n}\right\}$ to refer to a sequence of positive linear operator.

Definition 2.8 (Polynomial operator). A linear operator $L_{n}(f ; x)$ is called polynomial if its value for any function in its domain is an algebraic or a trigonometric polynomial. 


\subsubsection{Moduli of Smoothness}

Moduli of continuity and, in general, moduli of smoothness of order $k$ are a powerful tool to investigate the estimate of error of approximation by positive linear operators.

Definition 2.9 (Modulus of continuity). Modulus of continuity of $f \in C[a, b]$ is the function $\omega:[0, \infty) \rightarrow[0, \infty)$ defined by

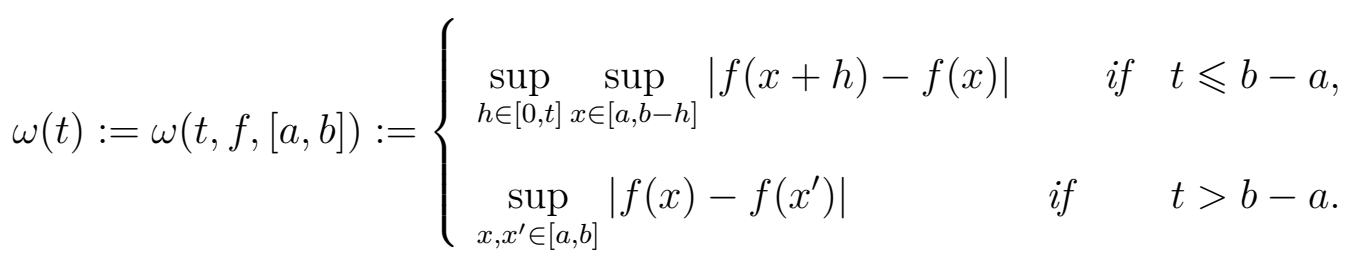

Definition 2.10 (Modulus of smoothness of order $k$ ). The modulus of smoothness of order $k$ of a function $f \in C[a, b]$ is the function $\omega_{k}:[0, \infty) \rightarrow[0, \infty)$ defined by

$$
\omega_{k}(t)=\omega_{k}(t, f,[a, b]):= \begin{cases}\sup _{h \in[0, t]} \sup _{x \in[a, b-k h]}\left|\Delta_{h}^{k}(f, x)\right| & \text { if } \quad t \leqslant \frac{b-a}{k}, \\ \omega_{k}\left(\frac{b-a}{k}, f,[a, b]\right) & \text { if } \quad t>\frac{b-a}{k} .\end{cases}
$$

Note that the modulus of smoothness of order 1 is the modulus of continuity, i.e., $\omega_{1}(t, f,[a, b])=\omega(t, f,[a, b])$. 


\section{Some Examples of Positive Linear}

\section{Operators}

In this chapter, we introduce and discuss a few well known positive linear operators:

Bernstein operator (1912), de la Vallée Poussin operator (1918), Fejér operator (1930), Jackson operator (1930), Kantorovich operator (1930), Bernstein-Chlodovsky operator (1937), Favard-Szász and Mirakjan operator (1941), Baskakov operator (1957), Bernstein-Durrmeyer operator (1967), Bleimann-Butzer-Hahn operator (1980), King operator (2003). Some of these operators (e.g. Bernstein) provide a simple tool for approximation of functions on compact intervals, while some others (e.g. Bernstein-Chlodovsky, Favard-Szász and Mirakjan) are appropriate for approximation of functions on semi-axes.

\subsection{Bernstein operator}

The Bernstein polynomial (Bernstein operator) was introduced by Sergei Bernstein in 1912. For a function $f \in C[0,1]$ and $n \in \mathbb{N}$, the Bernstein operator is defined as

$$
B_{n}(f ; x):=\sum_{k=0}^{n}\left(\begin{array}{l}
n \\
k
\end{array}\right) x^{k}(1-x)^{n-k} f\left(\frac{k}{n}\right)=\sum_{k=0}^{n} p_{n, k}(x) f\left(\frac{k}{n}\right)
$$


where

$$
p_{n, k}(x):=\left(\begin{array}{l}
n \\
k
\end{array}\right) x^{k}(1-x)^{n-k}, \quad 0 \leq k \leq n .
$$

In general, if $f$ is defined on $[a, b]$, we have

$$
B_{n}(f ; x ;[a, b]):=\frac{1}{(b-a)^{n}} \sum_{k=0}^{n}\left(\begin{array}{l}
n \\
k
\end{array}\right)(x-a)^{k}(b-x)^{n-k} f\left(a+\frac{k(b-a)}{n}\right) .
$$

It is obvious that $B_{n}(f ; 0)=f(0)$ and $B_{n}(f ; 1)=f(1)$, and is easy to verify that

1. $B_{n}(1 ; x)=1$,

2. $B_{n}(t ; x)=x$,

3. $B_{n}\left(t^{2} ; x\right)=\left(1-\frac{1}{n}\right) x^{2}+\frac{1}{n} x$.

It was shown by D. Stancu (see e.g. [52]) that

$$
f(x)-B_{n}(f ; x)=: R_{n}(f ; x)=-\frac{x(1-x)}{n} \sum_{k=0}^{n-1} p_{n-1, k}(x)\left[x, \frac{k}{n}, \frac{k+1}{n} ; f\right],
$$

and the identity (3.1) is now called the Stancu remainder for the Bernstein operator

In [52, Theorem 1], Stancu obtained a useful formula for the difference of two consecutive Bernstein polynomials in order to study monotonicity of the sequence $\left\{B_{n}\right\}$,

$$
B_{n+1}(f ; x)-B_{n}(f ; x)=-\frac{x(1-x)}{n(n+1)} \sum_{k=0}^{n-1} p_{n-1, k}(x)\left[\frac{k}{n}, \frac{k+1}{n+1}, \frac{k+1}{n} ; f\right] .
$$

Formula (3.2) is known as Aramă-Popoviciu-type Formula for Bernstein operator .

In Figure 3.1, we illustrate the approximation of the function $f(x)=1+\cos (7 \pi x)$, $x \in[0,1]$, by Bernstein polynomials $B_{n}$ for $n=10,50$ and 100 . One can see even from this figure that the rate of convergence of $B_{n}(f ; \cdot)$ to $f$ is very slow. 


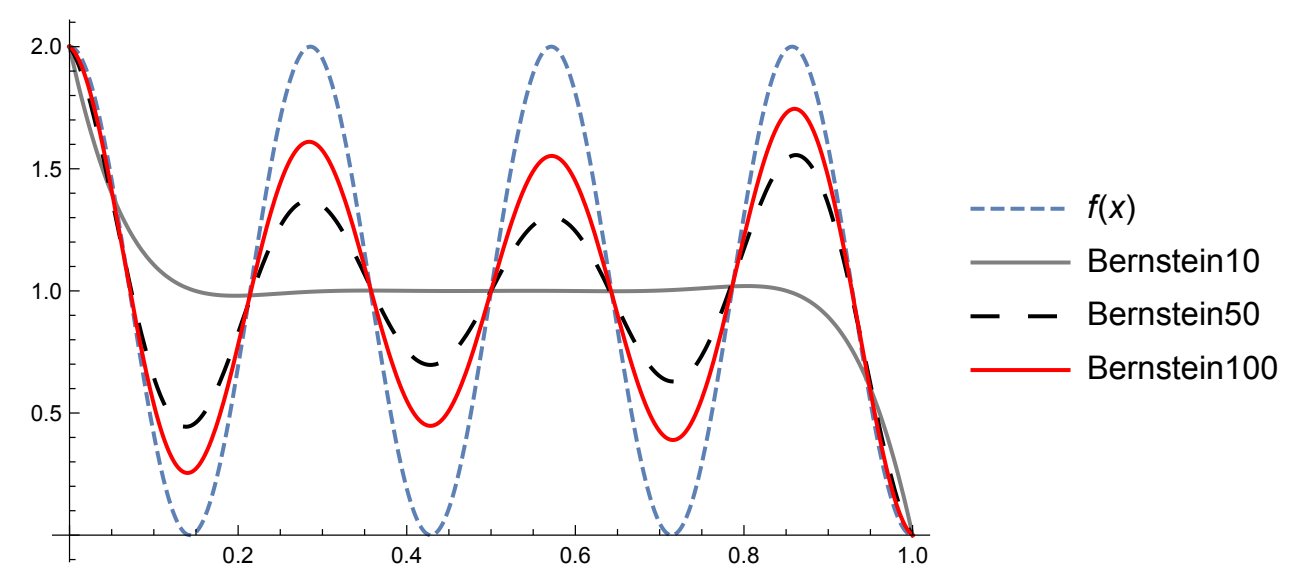

Figure 3.1: Approximation of $f(x)=1+\cos (7 \pi x)$ by Bernstein polynomials $B_{n}$ with $n=10,50,100$.

\section{2 de la Vallée Poussin operator}

In 1918, de la Vallée Poussin in his paper "Sur la meilleure approximation des fonctions d'une variable réelle par des expressions d'ordre donné " introduced the following operator for $f \in C[-\pi, \pi]$ :

$$
L_{n}(f ; x):=\frac{(n !)^{2}}{2 \pi(2 n) !} \int_{-\pi}^{\pi}\left(2 \cos \left(\frac{x-s}{2}\right)\right)^{2 n} f(s) d s
$$

which has the following properties for all $n \in \mathbb{N}$ :

1. $L_{n}(1 ; x)=1$,

2. $L_{n}(\cos t ; x)=\frac{n}{n+1} \cos x$,

3. $L_{n}(\sin t ; x)=\frac{n}{n+1} \sin x$

4. $L_{n}^{\prime}(f ; x)=L_{n}\left(f^{\prime} ; x\right)$ (see e.g. [44, p. 191]). 


\subsection{Fejér operator and Jackson operator}

Let $f$ be a real-valued, continuous and $2 \pi$-periodic function. For $p \in \mathbb{Z}^{+}$, the kernel $K_{n, p}(t)$ is defined by

$$
K_{n, p}(t):=c_{n, p}^{-1}\left(\frac{\sin \frac{n t}{2}}{\sin \frac{t}{2}}\right)^{2 p}
$$

where $c_{n, p}$ is the constant such that $\int_{-\pi}^{\pi} K_{n, p}(t) d t=1$. The kernel $K_{n, p}$ is called Stečkin's kernel (it was introduced by Stečkin in [53]).

A sequence of positive linear operators $\left\{L_{n, p}\right\}$ is defined by

$$
L_{n, p}(f ; x):=\int_{-\pi}^{\pi} f(x+t) K_{n, p}(t) d t .
$$

The Fejér operator ( $p=1$ in (3.3p) is a trigonometric polynomial of degree $n$ defined by

$$
\begin{aligned}
\sigma_{n}(f ; x): & =L_{n, 1}(f ; x) \\
& =\int_{-\pi}^{\pi} f(x+t) K_{n, 1}(t) d t \\
& =\frac{1}{2 \pi n} \int_{-\pi}^{\pi} f(x+t)\left(\frac{\sin \frac{n t}{2}}{\sin \frac{t}{2}}\right)^{2} d t .
\end{aligned}
$$

As an example, in Figures (3.2) and (3.3), we approximate function $h(x)=$ $g\left(\pi\left(\frac{x}{\pi}-\left\lfloor\frac{x}{\pi}\right\rfloor\right)\right)$, where

$$
g(x):=\left\{\begin{array}{lll}
\sqrt{3} x, & \text { if } \quad 0 \leq x \leq \frac{\pi}{2} \\
-\sqrt{3} x, & \text { if } \quad \frac{\pi}{2} \leq x \leq \pi .
\end{array}\right.
$$

by Fejér operators for $n=10$ and $n=100$, respectively.

The Fejér operator has the following properties: 


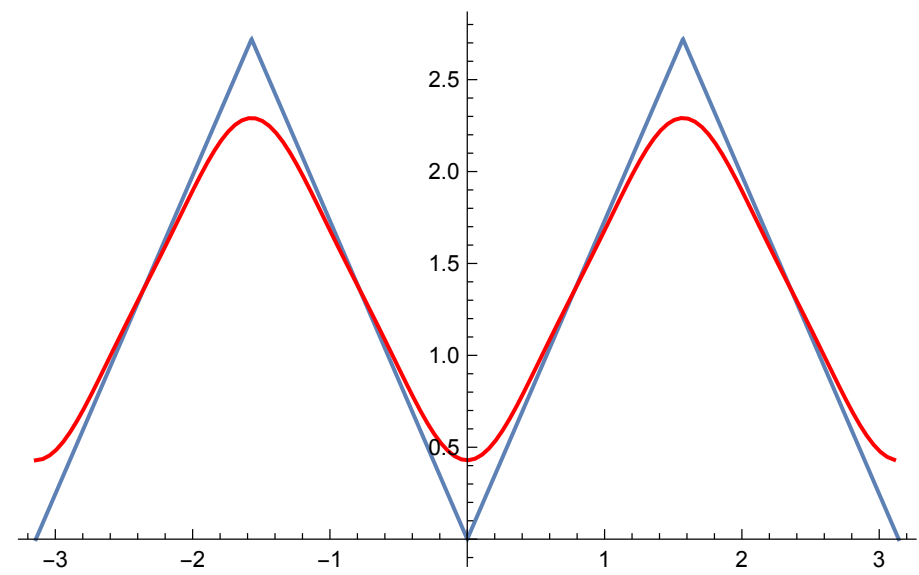

Figure 3.2: Approximation of $h(x)$ by Fejér operators for $n=10$.

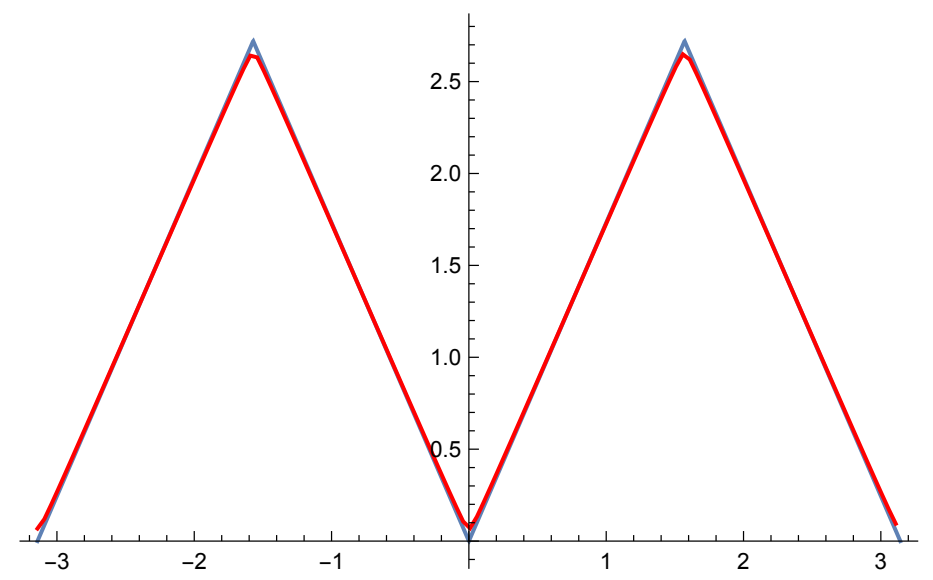

Figure 3.3: Approximation of $h(x)$ by Fejér operators for $n=100$. 
1. $\sigma_{n}(1 ; x)=1$,

2. $\sigma_{n}(\cos t ; x)=\frac{n-1}{n} \cos x$,

3. $\sigma_{n}(\sin t ; x)=\frac{n-1}{n} \sin x$.

If $p=2$, we have $L_{n, 2}(f ; x)=\mathcal{J}_{n}(f ; x)$, the so-called Jackson operator introduced by Jackson in 1930:

$$
\begin{aligned}
\mathcal{J}_{n}(f ; x): & =\int_{-\pi}^{\pi} f(x+t) K_{n, 2}(t) d t \\
& =\frac{3}{\pi}\left(4 n^{3}+2 n\right)^{-1} \int_{-\pi}^{\pi} f(x+t)\left(\frac{\sin \frac{n t}{2}}{\sin \frac{t}{2}}\right)^{4} d t
\end{aligned}
$$

The Jackson operator has the following properties:

1. $\mathcal{J}_{n}(1 ; x)=1$,

2. $\mathcal{J}_{n}(\cos t ; x)=\frac{2\left(n^{2}-1\right)}{2 n^{2}+1} \cos x$,

3. $\mathcal{J}_{n}(\sin t ; x)=\frac{2\left(n^{2}-1\right)}{2 n^{2}+1} \sin x$.

Example 3.1. For $p=3$, the $L_{n, p}$ operator defined in (3.4) is equal to

$$
\begin{aligned}
L_{n, 3}(f ; x): & =\int_{-\pi}^{\pi} f(x+t) K_{n, 3}(t) d t \\
& =\frac{1}{2 \pi}\left(\frac{20}{4 n+5 n^{3}+11 n^{5}}\right) \int_{-\pi}^{\pi} f(x+t)\left(\frac{\sin \frac{n t}{2}}{\sin \frac{t}{2}}\right)^{6} d t .
\end{aligned}
$$

For this operator we have,

1. $L_{n, 3}(1 ; x)=1$,

2. $L_{n, 3}(\cos t ; x)=\frac{-6-n^{2}+11 n^{4}}{4+5 n^{2}+11 n^{4}} \cos x$,

3. $L_{n, 3}(\sin t ; x)=\frac{-6-n^{2}+11 n^{4}}{4+5 n^{2}+11 n^{4}} \sin x$. 


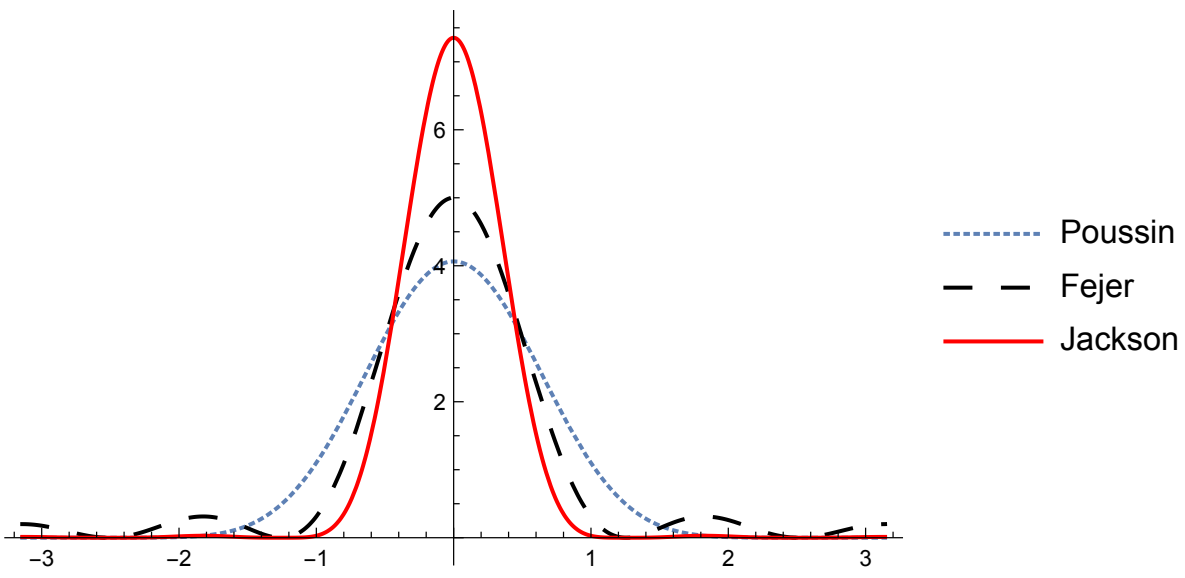

Figure 3.4: Kernels of convolution operators: Fejér, Jackson and de la Vallée Poussin.

\subsection{Kantorovich operator}

Bernstein operator s are not suitable for approximating discontinuous functions from $L_{p}$ (see for example Figure 3.6). In this regard, Kantorovich in 1930 in his paper "Sur certains développements suivant les polynômes de la forme de S. Bernstein" introduced an operator which is defined for functions which are integrable in the sense of Lebesgue.

Suppose $f(x)$ is an integrable function on $[0,1]$, and denote $F(x):=\int_{0}^{x} f(t) d t$. Then, the Kantorovich operator $K_{n}(f ; x)$ is defined as

$$
\begin{aligned}
K_{n}(f ; x) & =\frac{d}{d x} B_{n+1}(F ; x) \\
& =\sum_{k=0}^{n+1}\left(\begin{array}{l}
n \\
k
\end{array}\right) x^{k}(1-x)^{n-k}(n+1)\left\{F\left(\frac{k+1}{n+1}\right)-F\left(\frac{k}{n+1}\right)\right\} \\
& =\sum_{k=0}^{n+1}\left(\begin{array}{l}
n \\
k
\end{array}\right) x^{k}(1-x)^{n-k}(n+1)\left\{\int_{0}^{\frac{k+1}{n+1}} f(t) d t-\int_{0}^{\frac{k}{n+1}} f(t) d t\right\} \\
& =\sum_{k=0}^{n+1}\left(\begin{array}{l}
n \\
k
\end{array}\right) x^{k}(1-x)^{n-k}(n+1) \int_{\frac{k}{n+1}}^{\frac{k+1}{n+1}} f(t) d t .
\end{aligned}
$$

The Kantorovich operator has the following properties:

1. $K_{n}(1, x)=1$, 
2. $K_{n}(t, x)=\frac{n}{n+1} x+\frac{1}{2(n+1)}$,

3. $K_{n}\left(t^{2}, x\right)=\frac{n(n-1)}{(n+1)^{2}} x^{2}+\frac{2 n}{(n+1)^{2}} x+\frac{1}{3(n+1)^{2}}$.

\subsection{Bernstein-Chlodovsky operator}

A generalization of Bernstein polynomials for unbounded intervals was introduced by I. Chlodovsky in 1937 in [16]. Let $\left\{b_{n}\right\}$ be an increasing sequence such that

1. $b_{n} \in \mathbb{R}^{+}$,

2. $\lim _{n \rightarrow \infty} b_{n}=\infty$

3. $\lim _{n \rightarrow \infty} \frac{b_{n}}{n}=0$.

Let $f$ be a function defined on $[0, \infty)$ and bounded on every finite interval $[0, a] \subset$ $[0, \infty)$. For all $n \in \mathbb{N}$, Bernstein-Chlodovsky operator has the following form:

$$
C_{n}(f ; x)= \begin{cases}\sum_{k=0}^{n} p_{n, k}\left(\frac{x}{b_{n}}\right) f\left(b_{n} \frac{k}{n}\right) & x \in\left[0, b_{n}\right] \\ f(x) & x>b_{n} .\end{cases}
$$

where $p_{n, k}(x)$ are the fundamental Bernstein polynomials. For $b_{n}=1$, the operator $C_{n}(f ; x)$ is the classical Bernstein operator . The Bernstein-Chlodovsky operator has the following properties:

1. $C_{n}(1, x)=1$,

2. $C_{n}(t, x)=x$,

3. $C_{n}\left(t^{2}, x\right)=\left(1-\frac{1}{n}\right) x^{2}+\frac{b_{n}}{n} x$,

4. $C_{n}\left(t^{3}, x\right)=\frac{(n-1)(n-2)}{n^{2}} x^{3}+\frac{3(n-1)}{n^{2}} b_{n} x^{2}+\left(\frac{b_{n}}{n}\right)^{2} x$. 
5. $C_{n}\left(t^{4} ; x\right)=\frac{x^{4}}{n^{3}}\left(n^{3}-6 n^{2}+11 n-6\right)+6 b_{n}\left(n^{2}-3 n+2\right) \frac{x^{3}}{n^{3}}+7 b_{n}^{2}(n-1) \frac{x^{2}}{n^{3}}+\frac{x}{n^{3}} b_{n}^{3}$.

The condition $\lim _{n \rightarrow \infty} \frac{b_{n}}{n}=0$ is critical for convergence of $C_{n}(f ; x)$ to $f$. For instance, assume $b_{n}=n$ and $a \in \mathbb{R}$. Then, we have

$$
\begin{aligned}
\lim _{n \rightarrow \infty} C_{n}\left(e^{a t} ; x\right) & =\lim _{n \rightarrow \infty} \sum_{k=0}^{n}\left(\begin{array}{l}
n \\
k
\end{array}\right)\left(\frac{x}{n}\right)^{k}\left(1-\frac{x}{n}\right)^{n-k} e^{a k} \\
& =\lim _{n \rightarrow \infty} \sum_{k=0}^{n}\left(\begin{array}{l}
n \\
k
\end{array}\right)\left(\frac{x}{n} e^{a}\right)^{k}\left(1-\frac{x}{n}\right)^{n-k} \\
& =\lim _{n \rightarrow \infty}\left(1+\frac{x}{n}\left(e^{a}-1\right)\right)^{n} \\
& =e^{\left(e^{a}-1\right) x} \\
& \neq e^{a x}
\end{aligned}
$$

Thus, the operator does not converge to the function $e^{a x}$. Another example to support this is

$$
\lim _{n \rightarrow \infty} C_{n}\left(t^{2} ; x\right)=x^{2}+x \neq x^{2}
$$

\subsection{Favard-Szász-Mirakjan operator}

The Favard-Szász-Mirakjan operator was introduced by Mirakjan in 1941 and studied by Favard in 1944 and also by Szász in 1950. Let $M>0$ and $\alpha \in \mathbb{N}$, and define

$$
C_{M}[0, \infty):=\left\{f \in C[0, \infty):|f(x)| \leq M e^{\alpha x}\right\}
$$

The Favard-Szász-Mirakjan operator $\mathcal{F}_{n}: C_{M}[0, \infty) \rightarrow C[0, \infty)$ is

$$
\mathcal{F}_{n}(f ; x):=\sum_{k=0}^{\infty} e^{-n x} \frac{(n x)^{k}}{k !} f\left(\frac{k}{n}\right)
$$

This operator has the following properties:

1. $\mathcal{F}_{n}(1 ; x)=1$, 


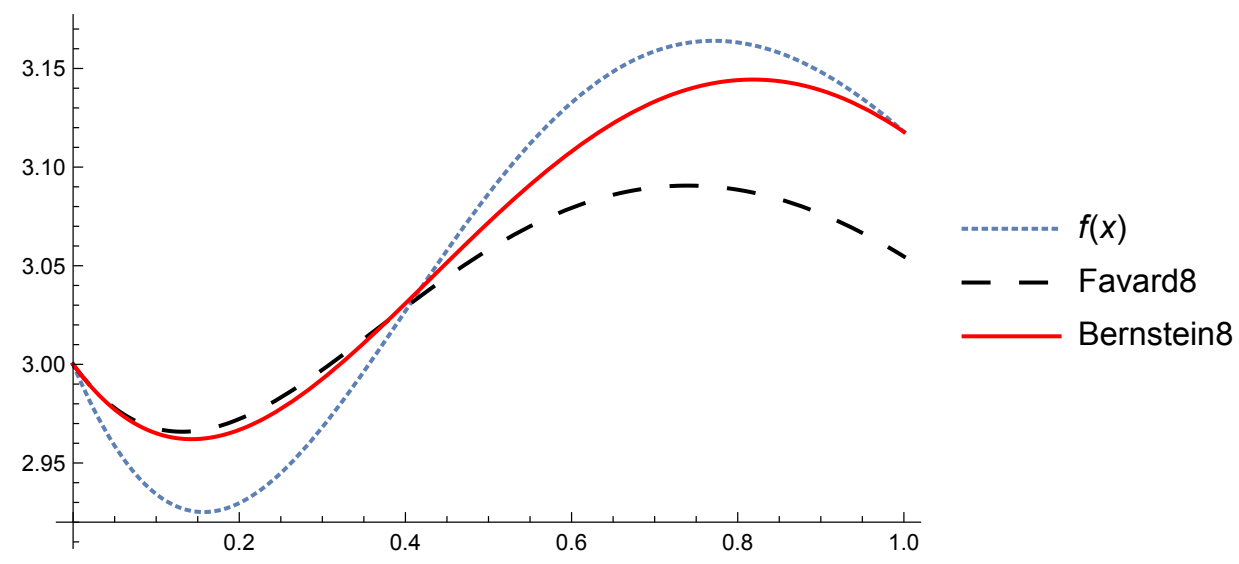

Figure 3.5: Approximation of $f(x)=2+e^{-x}+\frac{3 x^{2}}{\left(x^{2}+1\right)^{2}}$ by Bernstein and FavardSzász-Mirakjan operators for $n=8$.

2. $\mathcal{F}_{n}(t ; x)=x$,

3. $\mathcal{F}_{n}\left(t^{2} ; x\right)=x^{2}+\frac{1}{n} x$.

\subsection{Baskakov operator}

Let $C_{2}^{*}[0, \infty)$ be the weighted space of all continuous real-valued functions on $[0, \infty)$ such that $\lim _{x \rightarrow \infty} \frac{f(x)}{1+x^{2}}$ exists. For $f \in C_{2}^{*}[0, \infty)$ and $x \in[0, \infty)$, Baskakov operator, introduced by Baskakov in 1957 in [7], is defined by

$$
L_{n}(f ; x):=\sum_{k=0}^{\infty}\left(\begin{array}{c}
n+k-1 \\
k
\end{array}\right) \frac{x^{k}}{(1+x)^{n+k}} f\left(\frac{k}{n}\right) .
$$

Baskakov operator has the following properties:

1. $L_{n}(1 ; x)=1$,

2. $L_{n}(t ; x)=x$,

3. $L_{n}\left(t^{2} ; x\right)=x^{2}+\frac{x(1+x)}{n}$. 


\subsection{Durrmeyer operator}

In the paper "Une formule d'inversion de la Transformée de Laplace" published in 1967, J. L. Durrmeyer introduced the following operator in order to approximate Lebesgue integrable functions on $[0,1]$ :

$$
\mathcal{D}_{n}(f ; x):=\int_{0}^{1} f(u) K_{n}(x, u) d u, \quad x \in[0,1], \quad n \in \mathbb{N},
$$

where

$$
K_{n}(x, u):=(n+1) \sum_{k=0}^{n} p_{n, k}(x) p_{n, k}(u),
$$

and

$$
p_{n, k}(x):=\left(\begin{array}{l}
n \\
k
\end{array}\right) x^{k}(1-x)^{n-k}, \quad k=0,1, \ldots, n .
$$

Hence,

$$
\mathcal{D}_{n}(f ; x)=\sum_{k=0}^{n}\left(\begin{array}{l}
n \\
k
\end{array}\right) x^{k}(1-x)^{n-k} \int_{0}^{1}(n+1)\left(\begin{array}{l}
n \\
k
\end{array}\right) u^{k}(1-u)^{n-k} f(u) d u,
$$

and $\int_{0}^{1}(n+1)\left(\begin{array}{l}n \\ k\end{array}\right) u^{k}(1-u)^{n-k} f(u) d u$ in the Durrmeyer operator takes the place of $f(k / n)$ in the Bernstein operator. Hence, changing the value of $f$ at a single point will have no influence on the Durrmeyer operator while possibly significantly changing the Bernstein operator . For example, let

$$
f(x):=\left\{\begin{array}{lll}
x^{4}, & \text { if } \quad x \neq 1 / 2 \\
2, & \text { if } \quad x=1 / 2 .
\end{array}\right.
$$

Figure 3.6 illustrates approximation of $f$ by both of these operators.

The Durrmeyer operator has the following properties:

1. $\mathcal{D}_{n}(1 ; x)=1$, 


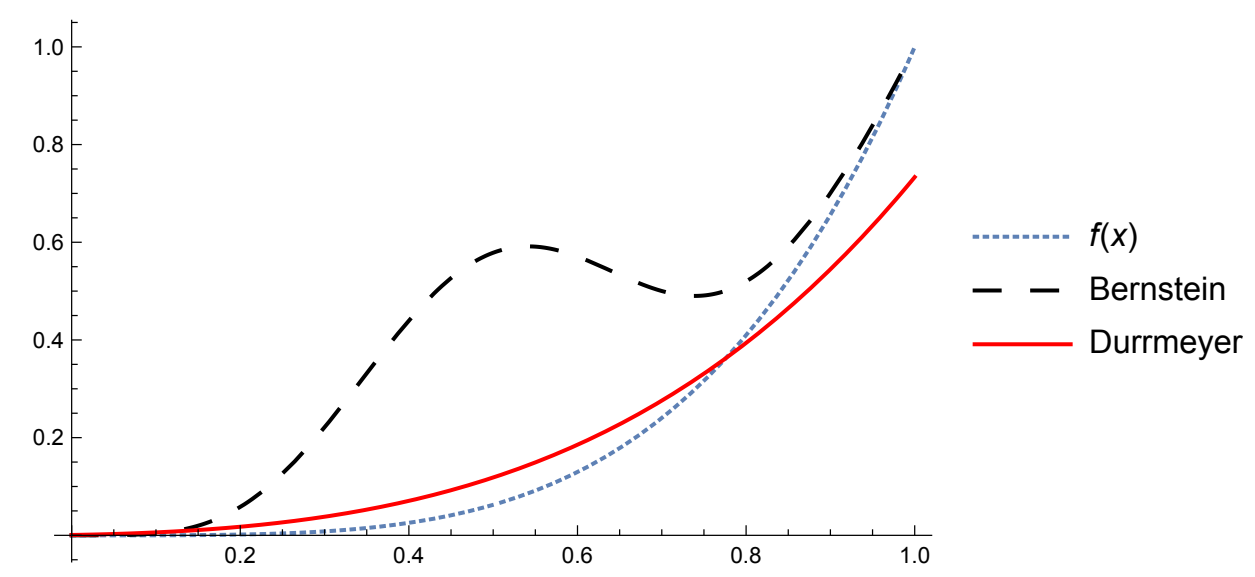

Figure 3.6: Approximation of $f(x)$ from $(3.8)$ by Bernstein and Durrmeyer operators for $n=10$.

2. $\mathcal{D}_{n}(t ; x)=\frac{n}{n+2} x+\frac{1}{n+2}$,

3. $\mathcal{D}_{n}\left(t^{2} ; x\right)=\frac{n(n-1)}{(n+2)(n+3)} x^{2}+\frac{4 n}{(n+2)(n+3)} x+\frac{2}{(n+2)(n+3)}$.

There are more nice properties for the Durrmeyer operator (for e.g. see [23, p. 121]). We define

$$
\mathcal{B}_{n}(f, x):=\int_{0}^{1} \frac{u^{n x}(1-u)^{n-n x}}{B(n x+1, n-n x+1)} f(u) d u
$$

note that, for $x=\frac{k}{n}$, we have

$$
\mathcal{B}_{n}\left(f ; \frac{k}{n}\right):=\int_{0}^{1} \frac{u^{k}(1-u)^{n-k}}{B(k+1, n-k+1)} f(u) \mathrm{d} u,
$$

verify that

$$
(n+1)\left(\begin{array}{l}
n \\
k
\end{array}\right)=\frac{1}{B(k+1, n-k+1)},
$$

and conclude

$$
\mathcal{B}_{n}\left(f ; \frac{k}{n}\right)=\int_{0}^{1}(n+1)\left(\begin{array}{l}
n \\
k
\end{array}\right) u^{k}(1-u)^{n-k} f(u) d u
$$


Hence,

$$
\mathcal{D}_{n}(f)=B_{n}\left(\mathcal{B}_{n}(f)\right)
$$

We will use this identity in the next chapters.

\subsection{Bleimann-Butzer-Hahn operator}

In 1980, Bleimann, Butzer and Hahn [9] introduced an operator of Bernstein-type on $C[0, \infty)$. If $f \in C[0, \infty)$ and $n \in \mathbb{N}$, then

$$
L_{n}(f ; x):=\sum_{k=0}^{n}\left(\begin{array}{l}
n \\
k
\end{array}\right) x^{k}(1+x)^{-n} f\left(\frac{k}{n-k+1}\right) .
$$

Like Favard-Szász-Mirakjan and Baskakov operators, Bleimann-Butzer-Hahn operator (briefly $\mathcal{B B H}$ ) is defined on $[0, \infty)$. However, we emphasize that the summation is now finite.

The $\mathcal{B B H}$ operator has the following properties:

$$
\begin{aligned}
& L_{n}(1 ; x)=1 \\
& L_{n}\left(\frac{t}{t+1} ; x\right)=\frac{n x}{(n+1)(x+1)}, \\
& L_{n}\left(\left(\frac{t}{t+1}\right)^{2} ; x\right)=\frac{n x(1+n x)}{(1+n)^{2}(x+1)^{2}} .
\end{aligned}
$$

\subsection{King operator}

In 2003, J. P. King [35] introduced an operator that preserves both $x^{2}$ and 1.

This operator $V_{n}: C[0,1] \rightarrow C[0,1]$ is defined as follows:

$$
V_{n}(f ; x):=\sum_{k=0}^{n}\left(\begin{array}{l}
n \\
k
\end{array}\right)\left(r_{n}(x)\right)^{k}\left(1-r_{n}(x)\right)^{n-k} f\left(\frac{k}{n}\right)
$$




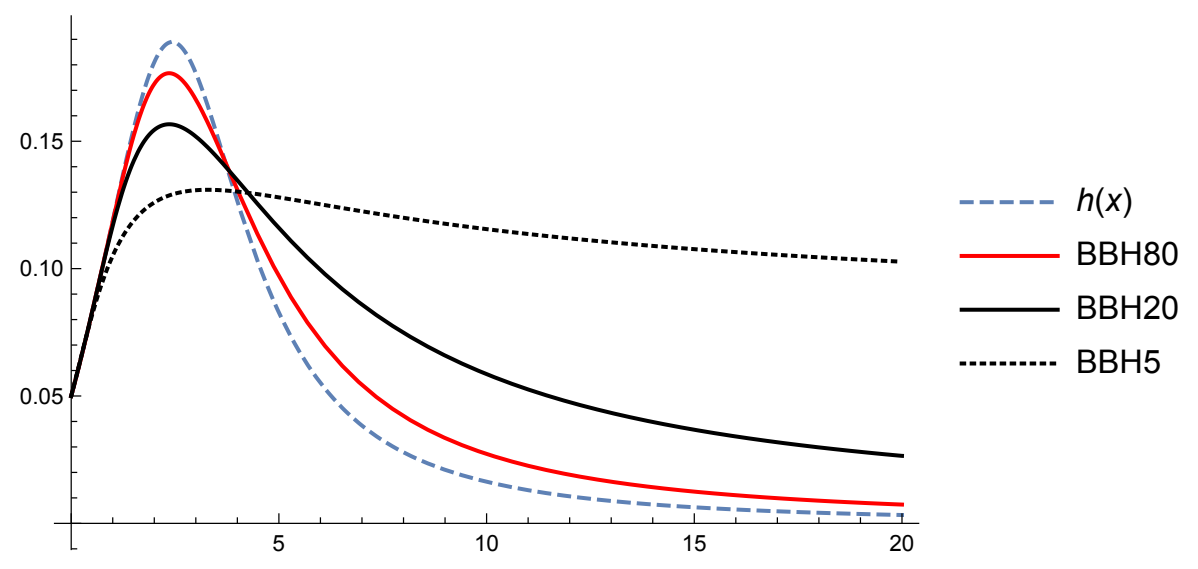

Figure 3.7: Approximation of $h(x)=\frac{(x+1)(x+5)}{x^{4}+100}$ by $\mathcal{B B H}$ operator for $n=5$, $n=20$, and $n=80$.

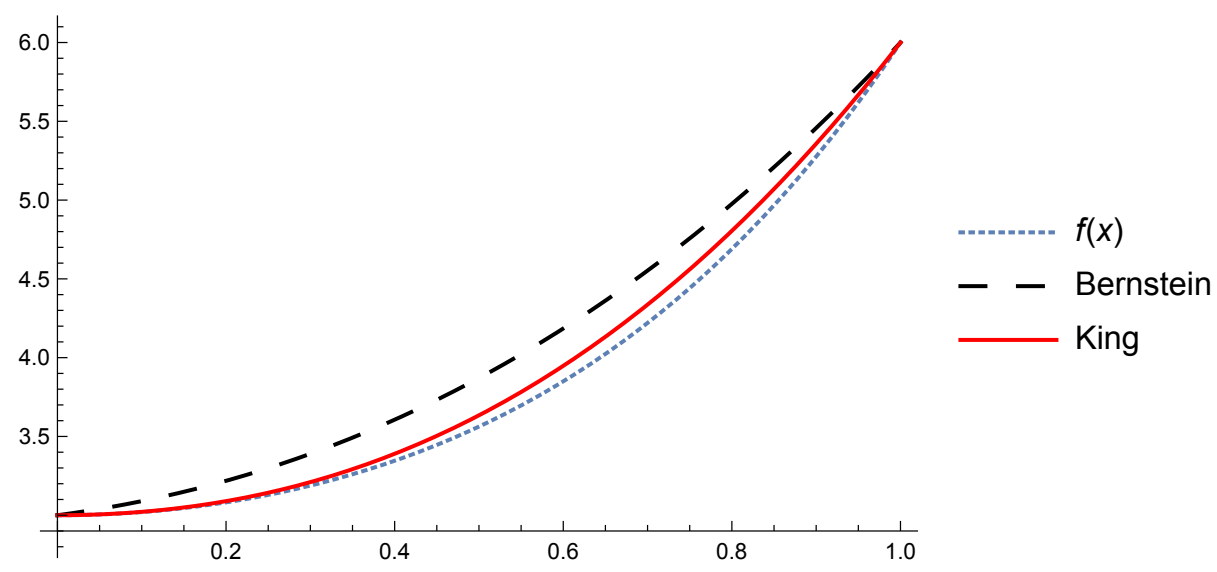

Figure 3.8: Approximation of $f(x)=2+\left(x^{2}+1\right)^{2}$ by Bernstein and King operators for $n=3$. 
where

$$
r_{n}(x):= \begin{cases}x^{2}, & \text { if } n=1, \\ \frac{-1+\sqrt{4 n(n-1) x^{2}+1}}{2(n-1)}, & \text { if } \quad n>1 .\end{cases}
$$

The King operator has these properties:

1. $V_{n}(1, x)=1$,

2. $V_{n}(t, x)=r_{n}(x)$,

3. $V_{n}\left(t^{2}, x\right)=x^{2}$.

Note that $V_{1}(f ; x)=\left(1-x^{2}\right) f(0)+x^{2} f(1)$, and it is clear that the King operator is not a polynomial operator for $n>1$, as we have $V_{n}(t ; x)=r_{n}(x)$. The King operator is similar to the Bernstein operator in the sense that it interpolates $f$ at the endpoints of interval. Indeed, since $r_{n}(0)=0$, we have

$$
\begin{aligned}
V_{n}(f ; 0) & =\sum_{k=0}^{n}\left(\begin{array}{l}
n \\
k
\end{array}\right)\left(r_{n}(0)\right)^{k}\left(1-r_{n}(0)\right)^{n-k} f\left(\frac{k}{n}\right) \\
& =f(0)+\sum_{k=1}^{n}\left(\begin{array}{l}
n \\
k
\end{array}\right)\left(r_{n}(0)\right)^{k} f\left(\frac{k}{n}\right)=f(0) .
\end{aligned}
$$

Similarly, $r_{n}(1)=1$ implies that $V_{n}(f ; 1)=f(1)$. 


\section{4}

\section{$K$-monotonicity Preservation by Positive Linear Operators}

The aim of this chapter is to discuss the shape preserving properties of positive linear operators. Let $\left\{L_{n}\right\}$ be a sequence of positive linear operators, and let $f$ be a $k$-monotone function (see Definition 2.3). We are interested in analyzing the impact of these operators on the function $f$. Under what conditions is $L_{n}(f ; x)$ itself $k$-monotone? We examine the relationship between $k$-monotonicity preservation and the strong variation diminishing property and total positivity of operators, and discuss results of Schoenberg and Karlin who had a significant contribution in this area.

As is well known, if $f^{(k)}$ exists on $[a, b]$, then $f^{(k)}(x) \geq 0$, for all $x \in[a, b]$, implies that the function $f$ is $k$-monotone on $[a, b]$.

We start with an overview of the impact of the Bernstein operator on convex functions. Relations (3.1) and (3.2) imply that, if $f$ is convex in $[0,1]$, then $B_{n}(f, x) \geq$ $B_{n+1}(f ; x)$ and $B_{n}(f, x) \geq f(x)$, respectively. Thus, for each convex function $f$ in $[0,1]$ and $n \in \mathcal{N}$,

$$
B_{n}(f, x) \geq B_{n+1}(f ; x) \geq f(x) .
$$


Furthermore, if $f$ is $k$-monotone on $[0,1]$ then, for each $0 \leq i \leq n-k$,

$$
\left[\frac{i}{n}, \ldots, \frac{i+k}{n} ; f\right] \geq 0
$$

Hence, since the $k$ th derivative of the Bernstein operator is given by

$$
B_{n}^{(k)}(f ; x)=\frac{k ! n !}{(n-k) ! n^{k}} \sum_{i=0}^{n-k}\left[\frac{i}{n}, \ldots, \frac{i+k}{n} ; f\right] p_{n-k, i}(x)
$$

we conclude that $B_{n}^{(k)}(f ; x) \geq 0$, i.e., the Bernstein operator preserves $k$-monotonicity.

Since it is not always easy to write the $k$ th derivative of an operator in terms of divided differences, another useful method that can be used to examine the $k$ monotonicity preservation of operators is that based on strong variation-diminishing (SVD) property of these operators.

\subsection{The SVD Property and $K$-monotonicity}

The concept of SVD was introduced by Schoenberg [49] in 1959.

Definition 4.1 (Strong variation-diminishing property, see e.g. [24, p. 4]). For $0 \leq$ $x_{0}<x_{1}<\cdots<x_{n} \leq 1$, let

$$
\nu_{[0,1]}\left(f ;\left\{x_{k}\right\}_{k=0}^{n}\right)
$$

denote the number of sign changes of $f(x)$ in the finite sequence $f\left(x_{0}\right), f\left(x_{1}\right), \ldots, f\left(x_{n}\right)$ where zeros are disregarded, and let $\nu_{[0,1]}(f)$ denote the number of sign changes of $f$ in $[0,1]$ :

$$
\nu_{[0,1]}(f)=\sup \left\{\nu_{[0,1]}\left(f ;\left\{x_{k}\right\}_{k=0}^{n}\right) \quad: \quad\left\{x_{k}\right\}_{k=0}^{n}\right\} .
$$

The operator $L: C[0,1] \rightarrow C[0,1]$ is said to be strong variation-diminishing if, for any $f \in C[0,1]$, we have

$$
\nu_{[0,1]}(L(f ; x)) \leq \nu_{[0,1]}(f)
$$


In 1958, Pólya and Schoenberg [46, Theorem 6] demonstrated that the Bernstein operator has the SVD property. Let $Z_{(0,1)}\left(B_{n}(f ; x)\right)$ denotes the number of zeros of $B_{n}(f ; x)$ in $(0,1)$. It is obvious that

$$
\nu_{[0,1]}\left(B_{n}(f)\right) \leq Z_{(0,1)}\left(B_{n}(f ; x)\right)
$$

Now, if $z=\frac{x}{1-x}, x \in(0,1)$, then $z \in(0, \infty)$ and we have

$$
\begin{aligned}
Z_{(0,1)}\left(B_{n}(f ; x)\right) & =Z_{(0,1)}\left(\frac{B_{n}(f ; x)}{(1-x)^{n}}\right) \\
& =Z_{(0, \infty)}\left(\sum_{k=0}^{n} f\left(\frac{k}{n}\right)\left(\begin{array}{l}
n \\
k
\end{array}\right) z^{n}\right) \\
& \leq \nu_{[0,1]}\left(f ;\left\{0, \frac{1}{n}, \ldots, 1\right\}\right) \\
& \leq \nu_{[0,1]}(f) .
\end{aligned}
$$

where $Z_{(0, \infty)}\left(\sum_{k=0}^{n} f\left(\frac{k}{n}\right)\left(\begin{array}{l}n \\ k\end{array}\right) z^{n}\right)$ denotes the number of zeros of $\sum_{k=0}^{n} f\left(\frac{k}{n}\right)\left(\begin{array}{l}n \\ k\end{array}\right) z^{n}$ in $(0, \infty)$.

Therefore, inequality (4.1) implies that

$$
\nu_{[0,1]}\left(B_{n}(f)\right) \leq \nu_{[0,1]}(f) .
$$

In 1998, Gavrea, Gonska, and Kacsó (25]) proved that the strong variationdiminishing, in the case of positive linear polynomial operators, implies preservation of $k$-monotonicity.

Theorem $4.2\left(\left[25\right.\right.$, Theorem 7]). Let $L_{n}: C[a, b] \rightarrow \Pi_{n}$ be a positive linear operator such that for $k=0,1, \ldots, n$, the degree of $L_{n}\left(t^{k} ; x\right)$ is equal to $k$.

If $L_{n}$ has the strongly variation diminishing property, i.e.,

$$
\nu_{[0,1]}\left(L_{n}(f ; x)\right) \leq \nu_{[0,1]}(f),
$$


then $L_{n}$ preserves the $k$-monotonicity, for $k=0,1, \ldots, n$.

Example 4.3 ([25, Theorem 2]). Recall that $\mathcal{D}_{n}(f)=B_{n}\left(\mathcal{B}_{n}(f)\right)$ (see (3.9)), and so

$$
\nu_{[0,1]}\left(\mathcal{D}_{n}(f ; x)\right)=\nu_{[0,1]}\left(B_{n}\left(\mathcal{B}_{n}(f ; x)\right)\right) .
$$

Since the Bernstein operator has the SVD property, we conclude

$$
\nu_{[0,1]}\left(B_{n}\left(\mathcal{B}_{n}(f ; x)\right)\right) \leq \nu_{[0,1]}\left(\mathcal{B}_{n}(f ; x)\right),
$$

and hence

$$
\nu_{[0,1]}\left(\mathcal{D}_{n}(f ; x)\right) \leq \nu_{[0,1]}\left(\mathcal{B}_{n}(f ; x)\right) .
$$

Substituting $\left(\frac{u}{1-u}\right)^{n}=t$ in the definition of $\mathcal{B}_{n}(f ; x)$, one has

$$
\begin{aligned}
\mathcal{B}_{n}(f ; x) & =\int_{0}^{1} u^{n x}(1-u)^{n-n x} f(u) \mathrm{d} u \\
& =\frac{1}{n} \int_{0}^{\infty} t^{x} \frac{t^{\frac{1}{n}-1}}{(1+\sqrt[n]{t})^{n+2}} f\left(\frac{\sqrt[n]{t}}{1+\sqrt[n]{t}}\right) d t .
\end{aligned}
$$

Theorem 1 in [25] implies that

$$
\nu_{[0,1]}\left(\mathcal{D}_{n}(f ; x)\right) \leq \nu_{[0,1]}(f) .
$$

Hence, the Durrmeyer operator has the strong variation-diminishing property.

Example 4.3 demonstrates that the Durrmeyer operator has the strong variationdiminishing property. In the next example, we will see that this operator preserves $k$-monotonicity. We also note that, in [2, Theorem 1, Remark 1 and 2] preservation of convexity by the Durrmeyer operator has been proved in a different way. 
Example 4.4. Derriennic [20] showed that

$$
\mathcal{D}_{n}\left(t^{k} ; x\right)=\frac{(n+1) !}{(n+k+1) !} \sum_{i=0}^{k}\left(\begin{array}{l}
k \\
i
\end{array}\right)\left(\begin{array}{l}
n \\
i
\end{array}\right) k ! x^{i}
$$

So, the Durrmeyer operator $\mathcal{D}_{n}$ transforms polynomials of degree $k, 0 \leq k \leq n$, into polynomials of degree $k$. Since the strong variation-diminishing property of the Durrmeyer operator is proved in Example 4.3, $\mathcal{D}_{n}$ satisfies conditions of the Theorem 4.2. thus, it preserves k-monotonicity.

\subsection{The Relation Between Total Positivity and $K$ - monotonicity}

In [29, Definition 3.4, 3.5], a totally positive matrix is defined as follows: A matrix is said to be totally positive if all its minors are nonnegative. A sequence $\left(\varphi_{0}, \varphi_{1}, \ldots, \varphi_{n}\right)$ of real-valued functions on an interval $I$ is totally positive if, for any set of points $0<x_{0}<x_{1}<\cdots<x_{n}$, the collocation matrix $\left(\varphi_{j}\left(x_{i}\right)\right)_{i, j=0}^{n}$ is totally positive. For instance, for $0<x_{0}<x_{1}<\cdots<x_{n}$,

$$
\left|\begin{array}{cccc}
1 & x_{0} & \ldots & x_{0}^{n} \\
\vdots & \vdots & \vdots & \vdots \\
1 & x_{n} & \ldots & x_{n}^{n}
\end{array}\right|>0
$$

is a total positive matrix. Thus, $\left(\varphi_{0}, \varphi_{1}, \ldots, \varphi_{n}\right)=\left(1, x, \ldots, x^{n}\right)$ is totally positive in any subinterval of $[0, \infty)$. To show that the kernel of the Bernstein operator is totally positive, we need the following lemma.

Lemma 4.5 ([58, Lemma 5.7]). Suppose that $\left(\varphi_{0}, \varphi_{1}, \ldots, \varphi_{n}\right)$ is totally positive on 
an interval $I$. If $\psi$ is an increasing function from an interval $J$ into $I$, then

$$
\left(\varphi_{0} \circ \psi, \varphi_{1} \circ \psi, \ldots, \varphi_{n} \circ \psi\right)
$$

is totally positive on $J$.

Assume that $\psi=\frac{x}{1-x}$. It is obvious that $\psi$ is an increasing function on $[0,1]$. Therefore, by Lemma 4.5, the composition of $\left(1, x, \ldots, x^{n}\right)$ and $\psi$ is totally positive, i.e.,

$$
\left(1, \frac{x}{1-x}, \ldots, \frac{x^{n}}{(1-x)^{n}}\right)
$$

is totally positive on $[0,1]$ and as a result by multiplying each function by $(1-x)^{n}$,

$$
\left((1-x)^{n}, x(1-x)^{n-1}, \ldots, x^{n}\right)
$$

is totally positive on $[0,1]$. This implies that the kernel of the Bernstein operator is totally positive.

The definition of total positivity of order $r$ is given as follows.

Definition 4.6 (Total positivity of order $r$, [32, p. 11]). Let $X$ and $Y$ be real intervals, and let $H(x, y)$ be defined on $X \times Y$ and suppose that, for each $i, x_{i} \in X$ and $y_{i} \in Y$. The function $H(x, y)$ is called a totally positive function of order $r$ if, for all $1 \leq m \leq r, x_{1}<x_{2}<\cdots<x_{m}$ and $y_{1}<y_{2}<\cdots<y_{m}$ we have

$$
H\left(\begin{array}{ccc}
x_{1} & \ldots & x_{m} \\
y_{1} & \ldots & y_{m}
\end{array}\right)=\left|\begin{array}{ccc}
H\left(x_{1}, y_{1}\right) & \ldots & H\left(x_{1}, y_{m}\right) \\
\vdots & \vdots & \vdots \\
H\left(x_{m}, y_{1}\right) & \ldots & H\left(x_{m}, y_{m}\right)
\end{array}\right| \geq 0
$$


For example, the following function $K(x, y)$ is total positive (see [32, p. 16])

$$
K(x, y)= \begin{cases}1, & a \leq x \leq y \leq b \\ 0, & \text { otherwise. }\end{cases}
$$

A few additional examples of totally positive functions are given in [32, pp. 1620]. Karlin [32, p. 25] showed that if $\left\{\varphi_{1}(x), \varphi_{2}(x), \ldots, \varphi_{n}(x)\right\}$ is a Descartes system, then the function

$$
K(x, y)=\varphi_{y}(x), \quad y=1,2, \ldots, n, \quad a<x<b
$$

is strictly totally positive of order $n$. Pinkus [45, pp. 87-90] demonstrated that

$$
K(x, y)=e^{x y}
$$

is totally positive, and it actually follows from Karlin [32, p. 18] that this statement can be made more general. Namely, if $\varphi(x)$ and $\psi(y)$ are increasing functions on sets $X$ and $Y$, respectively, then the kernel

$$
K(x, y)=e^{\varphi(x) \psi(y)}
$$

is totally positive on $X \times Y$.

In 1972, Lupaş introduced a Beta-type operator in his PhD dissertation "Die folge der Beta operatoren". The following example shows that the kernel of the Beta operator is totally positive. Recall that the kernel of this operator is

$$
\begin{aligned}
K(x, y): & =\frac{y^{n x}(1-y)^{n-n x}}{B(n x+1, n-n x+1)} \\
& =\frac{e^{n \log (1-y)} e^{n x(\log y-\log (1-y))}}{B(n x+1, n-n x+1)} .
\end{aligned}
$$


Example 4.7 ([6, Example 3.1]). By letting

$$
\Phi(x):=\frac{1}{B(n x+1, n-n x+1)}, \quad \Psi(y)=e^{n \log (1-y)},
$$

and

$$
K_{1}(x, y):=\exp (n x(\log y-\log (1-y)))
$$

the kernel of the Beta operator can be written as

$$
K(x, y)=\Phi(x) \Psi(y) K_{1}(x, y)
$$

Since $K_{1}(x, y)$ is totally positive (see Karlin [32, p. 18]), using [32, Theorem 2.1, p. 18], one concludes that the kernel of the Beta operator is totally positive as well.

Attalienti and Raşa [6, pp. 60-66] demonstrated that the kernels of the FavardSzász-Mirakjan, Baskakov, Durrmeyer and $n$-th Kantorovich operators are totally positive.

The relationship between totally positivity and convexity is investigated in [31]. Consider an operator $L$ such that

$$
L(f ; x):=\int_{Y} f(y) H(x, y) d y
$$

If the kernel $H(x, y)$ is totally positive of order 3 , the operator $L$ preserves constants, and $L(y ; x)=\alpha x+\beta$ where $\alpha>0$. In addition, if $f$ is a convex function, then $L(f ; x)$ is also convex.

Theorem 4.8 ([32, Proposition 3.2, p. 23]). Let $f$ be a convex function, and let $H(x, y)$ satisfy the following conditions:

$$
\text { 1. } \int_{Y} H(x, y) \mathrm{d} y=1
$$


2. $\int_{Y} y H(x, y) \mathrm{d} y=\alpha x+\beta$, where $x \in X$ and $\alpha>0$,

3. $H(x, y)$ is a totally positive function of order 3 , i.e.,

$$
H\left(\begin{array}{ccc}
x_{1} & x_{2} & x_{3} \\
y_{1} & y_{2} & y_{3}
\end{array}\right)=\left|\begin{array}{lll}
H\left(x_{1}, y_{1}\right) & H\left(x_{1}, y_{2}\right) & H\left(x_{1}, y_{3}\right) \\
H\left(x_{2}, y_{1}\right) & H\left(x_{2}, y_{2}\right) & H\left(x_{2}, y_{3}\right) \\
H\left(x_{3}, y_{1}\right) & H\left(x_{3}, y_{2}\right) & H\left(x_{3}, y_{3}\right)
\end{array}\right| \geq 0 .
$$

Then, the operator

$$
L(f ; x):=\int_{Y} f(y) H(x, y) d y
$$

is convex.

As an example, we can show that the Durrmeyer operator preserves convexity. The kernel of the Durrmeyer operator is

$$
H_{n}(x, y)=(n+1) \sum_{k=0}^{n}\left(\begin{array}{l}
n \\
k
\end{array}\right)\left(\begin{array}{l}
n \\
k
\end{array}\right)(x y)^{k}((1-x)(1-y))^{n-k}
$$

and we check the conditions of Theorem 4.8. We have

$$
\int_{Y} H_{n}(x, y) \mathrm{d} y=\int_{0}^{1}(n+1) \sum_{k=0}^{n}\left(\begin{array}{l}
n \\
k
\end{array}\right) x^{k}(1-x)^{n-k}\left(\begin{array}{l}
n \\
k
\end{array}\right) y^{k}(1-y)^{n-k} d y=1
$$

and

$$
\begin{aligned}
\int_{Y} y H_{n}(x, y) \mathrm{d} y & =\int_{0}^{1}(n+1) \sum_{k=0}^{n}\left(\begin{array}{l}
n \\
k
\end{array}\right) x^{k}(1-x)^{n-k}\left(\begin{array}{l}
n \\
k
\end{array}\right) y^{k+1}(1-y)^{n-k} d y \\
& =\frac{n}{2+n} x+\frac{1}{2+n}
\end{aligned}
$$

where $\alpha=\frac{n}{2+n}>0$.

Finally, since it follows from Attalienti and Raşa [6, Example 3.2] that the kernel of the Durrmeyer operator is totally positive, Theorem 4.8 implies that the Dur- 
rmeyer operator preserves convexity.

Karlin generalized Theorem 4.8 in [32, Proposition 3.3, p. 24].

Theorem 4.9 ([32, Proposition 3.3, p. 24]). Let $X$ and $Y$ be open intervals and $P_{m}(y)$ be an arbitrary polynomial of degree at most $m$. Assume that for $x \in X$ and all $m=0,1,2, \ldots, k-1$,

$$
\int_{Y} H(x, y) y^{m} d y=a_{m} x^{m}+P_{m-1}(x)
$$

where $a_{m}>0$, and suppose that $H(x, y)$ be totally positive of order $k+1$. If $f$ is k-monotone, then

$$
L(f ; x):=\int_{Y} f(y) H(x, y) d y
$$

is k-monotone.

We apply this theorem to the Durrmeyer operators. From 4.2, one has

$$
\int_{Y} H_{n}(x, y) y^{m} d y=\frac{(n+1) ! m !}{(n+m+1) !}\left(\begin{array}{c}
n \\
m
\end{array}\right) x^{m}+P_{m-1}(x)
$$

where

$$
P_{m-1}(x)=\frac{(n+1) ! m !}{(n+m+1) !} \sum_{i=0}^{m-1}\left(\begin{array}{c}
m \\
i
\end{array}\right)\left(\begin{array}{c}
n \\
i
\end{array}\right) x^{i},
$$

and the total positivity of the Durrmeyer kernel has proved in [6, Example 3.2]. Therefore, the above theorem the Durrmeyer operators preserves $k$-monotonicity.

\subsection{Approximation of Convex Functions by Posi- tive Linear Operators}

In this section, we discuss the approximation of convex functions by operators introduced previously. 
We first need to define centered Bernstein-type operator. These kinds of operators preserve affine functions and can be written in terms of the mathematical expectation of an appropriate random variable.

Definition 4.10 ([18, p. 1107]). Let I be an interval, and for each $x \in I, \xi(x)$ be an I-value random variable. Let $\mathcal{L}$ stand for the domain of an operator $L$, that is, the set of all real functions on I for which Lf is defined. A centered Bernstein-type operator is a positive linear operator which preserves affine functions and can be expressed in terms of a mathematical expectation, i.e.,

$$
L(f ; x):=\mathbb{E} f(\xi(x)), \quad \text { for all } f \in \mathcal{L}, \text { and } x \in I,
$$

where $\mathbb{E}$ denotes the mathematical expectation.

Recall that the mathematical expectation of a continuous random variable $\xi(x)$ with distribution density $f(x)$ is $\mathbb{E} \xi(x)$ defined by

$$
\mathbb{E} \xi(x)=\int_{-\infty}^{\infty} x f(x) d x
$$

Similarly, mathematical expectation of a discrete random variable $\xi(x)$ is defined by

$$
\mathbb{E} \xi(x)=\sum_{k} x_{k} p_{k}
$$

where $x_{k}$ and $p_{k}$ are possible values of $\xi(x)$ and corresponding probabilities of these values, respectively.

Let $L$ be a positive linear centered Bernstein-type operators over an interval $I$ of the real line and $f \in \mathcal{L}$. By definition, $L(f ; x):=\mathbb{E} f(\xi(x))$, where $\xi(x)$ is a random variable. By Jensen's inequality, if $\xi(x)$ is a random variable and $f$ is a 
convex function, then we have

$$
f(\mathbb{E}(\xi(x))) \leq \mathbb{E} f(\xi(x)) .
$$

On the other hand, operator $L$ preserves affine functions. Thus,

$$
L(t ; x)=x=\mathbb{E}(\xi(x)) .
$$

As a conclusion, we have

$$
L(f ; x)=\mathbb{E} f(\xi(x)) \geq f(\mathbb{E}(\xi(x)))=f(x) .
$$

Relation 4.4 implies that centered Bernstein-type operators approximate convex functions from above. If $L$ and $M$ are two positive linear centered Bernstein-type operators over an interval $I$ of the real line, then if for all convex functions $f \in \mathcal{L}$ in the domains of $L$ and $M$ if we have

$$
L(f) \geq M(f) \geq f,
$$

then $M$ approximates $f$ better than $L$.

Does the relation (4.5) implies that $L-M$ is a positive operator? Generally, $L-M$ is not positive. As an example, let $f(x)=1-x^{2}$. For $x \in[0,1], f \geq 0$. Let $M$ and $L$ be Bernstein and Favard-Szász-Mirakjan operators, respectively. Thus,

$$
\begin{aligned}
(L-M)(f ; x) & =\left(\mathcal{F}_{n}-B_{n}\right)\left(1-t^{2} ; x\right) \\
& =\mathcal{F}_{n}\left(1-t^{2} ; x\right)-B_{n}\left(1-t^{2} ; x\right) \\
& =\left(1-x^{2}-\frac{x}{n}\right)-\left(1-x^{2}+\frac{x^{2}}{n}-\frac{x}{n}\right) \\
& =-\frac{x^{2}}{n} \leq 0 .
\end{aligned}
$$




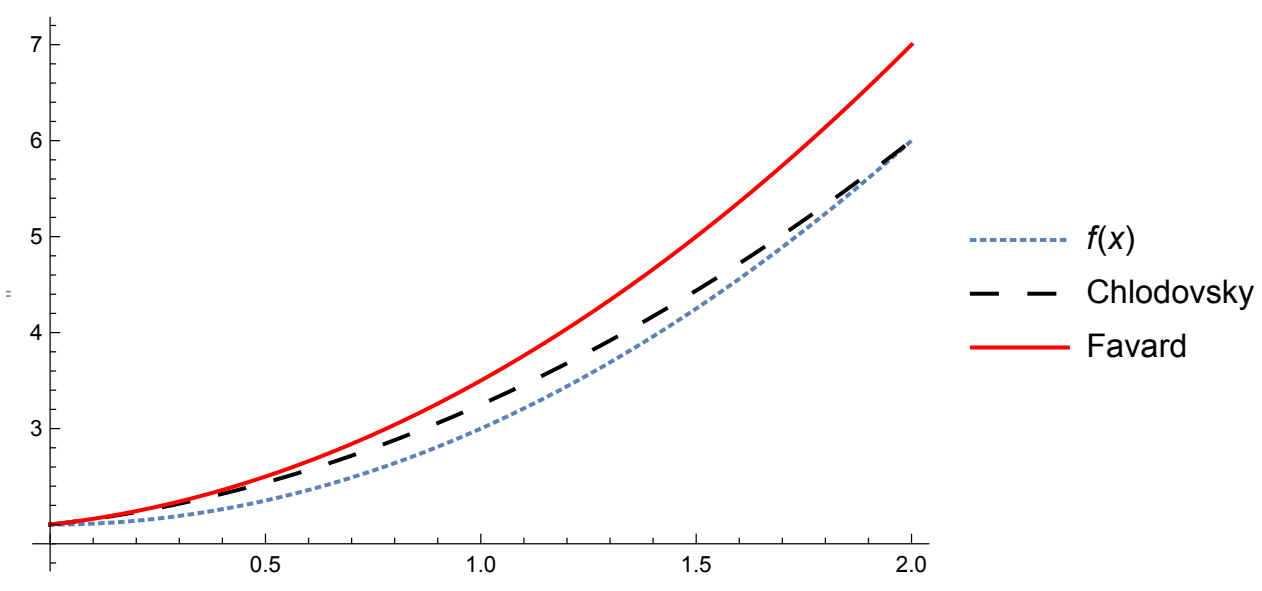

Figure 4.1: Approximation of $f(x)=2+x^{2}$.

For example, the following special Bernstein-Chlodovsky operator $C_{n m}$ approximates convex functions from the domain of $\mathcal{F}_{n}$ (Favard-Szász-Mirakjan operator) better than $\mathcal{F}_{n}$ :

$$
C_{n m}(f ; x)= \begin{cases}\sum_{k=0}^{n m} p_{n m, k}\left(\frac{x}{m}\right) f\left(\frac{k}{n}\right), & \text { if } x \in[0, m], \\ f(x), & \text { if } x>m .\end{cases}
$$

This fact follows from the following theorem.

Theorem 4.11 ([18, Theorem 2]). For each convex function $f$ in the domain of $\mathcal{F}_{n}$, we have

$$
\mathcal{F}_{n}(f) \geq C_{n m}(f)
$$

In Figure 4.1, we illustrate the approximation of $f(x)=2+x^{2}$ on $[0,2]$ by the Favard-Szász-Mirakjan operator for $n=2$ and by $C_{n m}(f ; x)$ defined in (4.6) for $n=m=2$. We see that, in line with the result of Theorem 4.11, the BernsteinChlodovsky operator approximates $f(x)$ better than Favard-Szász-Mirakjan operator.

Cal and Cárcamo [18, Theorem 1] showed that if $L_{1}$ (which is the composition 


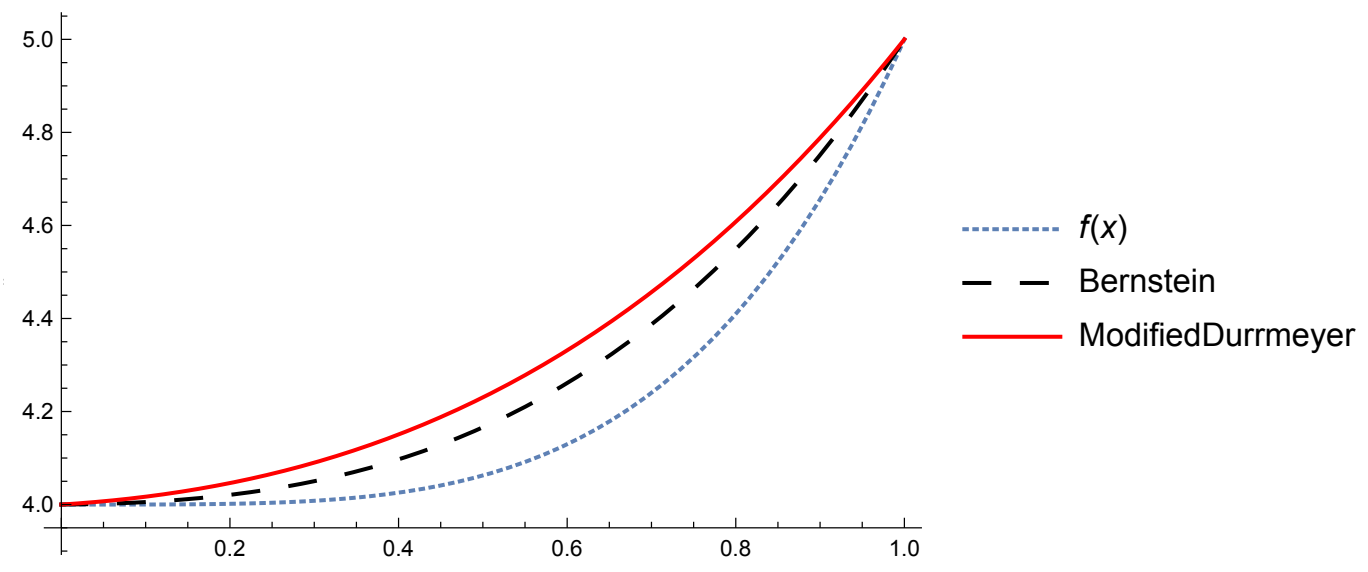

Figure 4.2: Approximation of function $f(x)=4+x^{4}$ by the Bernstein operator and modified Durrmeyer operator.

of two centered Bernstein-type operators $L_{2}$ and $L_{3}$ ) is a centered Bernstein-type operator, then $L_{1}$ does not approximate convex functions better than $L_{2}$.

Theorem 4.12 ([18, Theorem 1]). Assume that $L_{1}=L_{2} \circ L_{3}$, where $L_{1}, L_{2}$ and $L_{3}$ are centered Bernstein-type operators over the same interval, and let $\mathcal{L}_{c x}$ be the set of all convex functions in the domains of these three operators. Then, we have

$$
L_{1} f \geq L_{2} f, \quad f \in \mathcal{L}_{c x}
$$

For instance, a modified Durrmeyer operator $U_{n}$ is the composition of Bernstein and Beta operators, i.e., $U_{n}=B_{n} \circ B_{n}^{*}$, where

$$
U_{n}(f ; x)=f(0)(1-x)^{n}+f(1) x^{n}+(n-1) \sum_{k=1}^{n-1} p_{n, k}(x) \int_{0}^{1} f(u) p_{n-2, k-1}(u) d u
$$

and

$$
B_{n}^{*}(f ; x)= \begin{cases}\int_{0}^{1} f(u) \frac{u^{n x-1}(1-u)^{n-n x-1}}{B(n x, n-n x)} d u, & \text { if } x \in(0,1), \\ f(x), & \text { if } x=0 \text { or } x=1 .\end{cases}
$$

Therefore, by Theorem 4.12 , one has $U_{n}(f) \geq B_{n}(f)$ for all $f \in \mathcal{L}_{c x}$, and so the Bern- 


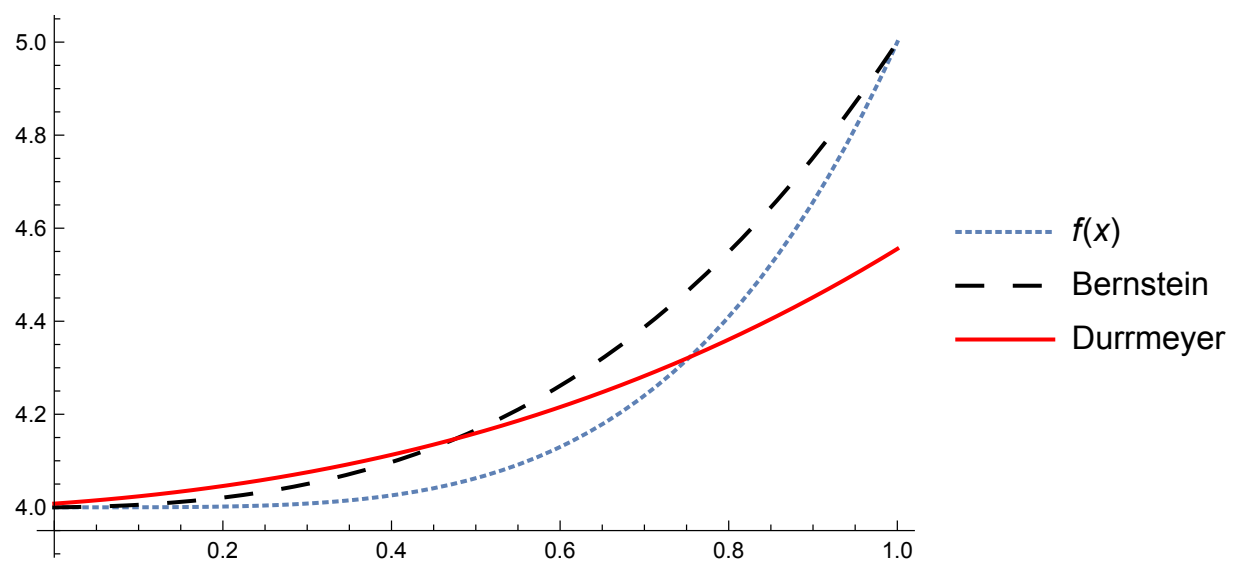

Figure 4.3: Approximation of function $f(x)=4+x^{4}$ by Bernstein operator and Durrmeyer operator for $n=4$.

stein operator approximates convex functions better than the modified Durrmeyer operator $U_{n}$ (this is illustrated in Figure 4.2).

We note that one must be careful when applying Theorem 4.12. It follows from (3.9) that the Durrmeyer operator can be written as the composition $\mathcal{D}_{n}=B_{n} \circ \mathcal{B}_{n}$. However, as can be seen from Figure 4.3, the Durrmeyer operator does not approximate the convex function $f$ better than the Bernstein operator , i.e., the inequality $\mathcal{D}_{n}(f) \geq B_{n}(f)$ does not hold for all $f \in \mathcal{L}_{c x}$. This happens because the Durrmeyer operator is not a centered Bernstein-type operator, and so this requirement in the theorem is essential and cannot be dropped.

Now, let $\mathcal{L}_{n}$ be the class of all positive polynomial operators $L$ on $C[0,1]$ that preserve affine functions and have the form

$$
L_{n}(f ; x)=\sum_{k=0}^{n}\left(\begin{array}{l}
n \\
k
\end{array}\right) x^{k}(1-x)^{n-k} \int_{0}^{1} f d \mu_{k, n}
$$

where $\mu_{k, n}$ is a positive Borel measure on $[0,1]$. Denote by $\delta_{x}$ the Dirac delta probability, the Bernstein operator is obtained by taking $\mu_{k, n}$ as $\delta_{\frac{k}{n}}$. Cárcamo and de la Cal demonstrated that it is impossible to approximate any real-valued convex function in $C[0,1]$ by any operator $L \in \mathcal{L}_{n}$ better than Bernstein operator . 
Theorem 4.13 ([17, Theorem 2]). Let $n \geq 2$ and $f$ be any real-valued convex function in $C[0,1]$. If $L_{n} \in \mathcal{L}_{n}$, then

$$
f \leq B_{n}(f) \leq L_{n}(f)
$$

We finish this section noting that it can be seen from Figure 3.8 that the convex function $f(x)=2+\left(x^{2}+1\right)^{2}$ can be approximated by the King operator (which does not preserve affine functions) better than by the Bernstein operator . 


\section{5}

\section{Convergence Theory of Positive Linear Operators}

Suppose we have a sequence of positive linear operator $\left\{L_{n}(f ; x)\right\}$. Does this sequence converge to the function $f(x)$ and, if it does, how fast is this convergence? This chapter investigates the basic theorems on convergence of positive linear operators.

The Bohman-Korovkin Theorem provides necessary and sufficient conditions that guarantee the convergence of sequences of linear positive operators.

In this chapter, after introducing this and other analogous theorems, we apply them to several well known positive linear operators. In Section 5.4, we discuss Voronovskaja formulas in order to study the asymptotic behavior of positive linear operators. This concept was studied for the first time in 1932 by Voronovskaja who was a student of Bernstein.

\subsection{The Bohman-Korovkin Theorem}

Let $\left\{L_{n}\right\}$ be a sequence of positive linear operators and $f$ be a continuous function defined on a closed interval. In this section, we study sufficient conditions to 
guarantee that $\left\{L_{n}(f)\right\}$ converges uniformly to $f$.

Korovkin ${ }^{1}$ and Bohman [11, pp. 43-45] established one of the most powerful criteria to find out if a sequence of positive linear operators tends to the identity operator in the space $C[a, b]$. They demonstrated (separately) that, for any $f \in$ $C[a, b]$, we have uniform convergence of the sequence $\left\{L_{n}(f)\right\}$ to $f$ if and only if $\lim _{n \rightarrow \infty} L_{n}\left(t^{j} ; x\right)=x^{j}$ uniformly for $j=0,1,2$.

Theorem 5.1 ([37, Theorem 3, p. 14]). If the three conditions

$$
\begin{aligned}
& L_{n}(1 ; x)=1+\alpha_{n}(x), \\
& L_{n}(t ; x)=x+\beta_{n}(x), \\
& L_{n}\left(t^{2} ; x\right)=x^{2}+\gamma_{n}(x) .
\end{aligned}
$$

are satisfied for the sequence of linear positive operators $L_{n}(f ; x)$, where $\alpha_{n}(x), \beta_{n}(x)$ and $\gamma_{n}(x)$ converge uniformly to zero in the interval $a \leq x \leq b$, then the sequence $L_{n}(f ; x)$ converges uniformly to the function $f(x)$ in this interval, if $f(t)$ is continuous in the interval $[a, b]$

Theorem 5.1 is now known as the Bohman-Korovkin Theorem and applies to positive linear operators which are defined for continuous functions on $[a, b]$. The Bohman-Korovkin Theorem guarantees the uniform convergence of the sequence of linear positive operators if conditions 5.1 are satisfied. It is worth mentioning that it is not enough to assume that the sequence $\left\{L_{n}\left(e_{j}\right)\right\}$ is convergent in $C[0,1]$ for $j=0,1,2$, where $e_{j}$ are the Korovkin test functions. We need to assume uniform convergence to $e_{j}$. Heping [30, pp. 260-261] constructed a sequence of positive linear operator such that $\left\{L_{n}\left(e_{j}\right)\right\}$ converges in $C[0,1]$ for $j=0,1,2$ (but at least one

\footnotetext{
${ }^{1}$ Pavel Petrovich Korovkin (1913-1985) was a Russian mathematician. He received his doctorate in 1939 with a thesis on orthogonal polynomials. His main areas of work were orthogonal polynomials, approximation theory and potential theory. At the beginning of the 1950s, he turned to functional analysis and studied the stability of the outer Dirichlet problem and the convergence behavior of linear positive operators on spaces of continuous functions. https://de.wikipedia.org/wiki/Pawel_Petrowitsch_Korowkin
} 
of them does not converge to $e_{j}$ ), and demonstrated that there exists a function $f \in C[0,1]$ such that $\left\{L_{n}(f)\right\}$ does not converge in $C[0,1]$.

Remark 5.2. Korovkin [37, Corollary 2, p.41] showed that it is impossible to replace the three functions $1, x, x^{2}$ by any two functions $f_{0}(x)$ and $f_{1}(x)$.

In the periodic case, Korovkin test functions are replaced by $t_{0}=1, t_{1}=\cos x$ and $t_{2}=\sin x$.

Theorem 5.3 ([37, Theorem 4, p. 17]). If the three conditions

$$
\begin{aligned}
& L_{n}(1 ; x)=1+\alpha_{n}(x), \\
& L_{n}(\cos t ; x)=\cos x+\beta_{n}(x), \\
& L_{n}(\sin t ; x)=\sin x+\gamma_{n}(x) .
\end{aligned}
$$

are satisfied for the sequence of linear positive operators $L_{n}(f ; x)$, where $\alpha_{n}(x), \beta_{n}(x)$ and $\gamma_{n}(x)$ converge uniformly to zero in the interval $[a, b]$, then the sequence $L_{n}(f ; x)$ converges uniformly to the function $f(x)$ in this interval where the function $f(t)$ is bounded, has period $2 \pi$, is continuous in the interval $[a, b]$.

We now illustrate Theorem 5.2 by considering the following example.

Example 5.4. Recall that $L_{n, 3}$ from Example 3.1 is defined as follows

$$
L_{n, 3}(f ; x)=\frac{1}{2 \pi}\left(\frac{20}{4 n+5 n^{3}+11 n^{5}}\right) \int_{-\pi}^{\pi} f(x+t)\left(\frac{\sin \frac{n t}{2}}{\sin \frac{t}{2}}\right)^{6} d t
$$

Then,

1. $\lim _{n \rightarrow \infty} L_{n, 3}(1 ; x)=1$,

2. $\lim _{n \rightarrow \infty} L_{n, 3}(\cos t ; x)=\lim _{n \rightarrow \infty}\left(\cos x+\frac{-6 n^{2}-10}{4+5 n^{2}+11 n^{4}} \cos x\right)=\cos x$, 
3. $\lim _{n \rightarrow \infty} L_{n, 3}(\sin t ; x)=\lim _{n \rightarrow \infty}\left(\sin x+\frac{-6 n^{2}-10}{4+5 n^{2}+11 n^{4}} \sin x\right)=\sin x$.

Since $\cos x$ and $\sin x$ are bounded, we have $\lim _{n \rightarrow \infty}\left(\frac{-6 n^{2}-10}{4+5 n^{2}+11 n^{4}} \cos x\right)=0$, and $\lim _{n \rightarrow \infty}\left(\frac{-6 n^{2}-10}{4+5 n^{2}+11 n^{4}} \sin x\right)=0$, uniformly.

Thus, conditions of Theorem 5.3 are fulfilled and so

$$
\lim _{n \rightarrow \infty} L_{n, 3}(f ; x)=f(x) \quad \text { uniformly }
$$

Now, let $\left\{\kappa_{n}\right\}$ be a sequence of positive kernels (i.e., $\kappa_{n} \geq 0$ a.e. on $\mathbb{R}$ ) which generates a sequence of positive linear operators $\left\{L_{n}\right\}$ on $L_{2 \pi}^{1}(\mathbb{R})$ defined by

$$
\begin{aligned}
L_{n}(f ; x): & =\left(f * \kappa_{n}\right)(x) \\
& =\frac{1}{2 \pi} \int_{-\pi}^{\pi} f(x-t) \kappa_{n}(t) d t \\
& =\frac{1}{2 \pi} \int_{-\pi}^{\pi} f(t) \kappa_{n}(x-t) d t .
\end{aligned}
$$

For $n \geq 1$, we define $\beta_{n}$ as

$$
\beta_{n}:=\frac{1}{2 \pi} \int_{-\pi}^{\pi} \sin ^{2}\left(\frac{t}{2}\right) \kappa_{n}(t) d t
$$

For instance, for the kernel of the Fejér operator, we have

$$
\begin{aligned}
\beta_{n} & =\frac{1}{2 \pi n} \int_{-\pi}^{\pi} \sin ^{2}\left(\frac{t}{2}\right)\left(\frac{\sin \frac{n t}{2}}{\sin \frac{t}{2}}\right)^{2} d t \\
& =\frac{1}{2 \pi n} \int_{-\pi}^{\pi}\left(\sin \frac{n t}{2}\right)^{2} d t \\
& =\frac{1}{2 n} .
\end{aligned}
$$

Theorem 5.5 ([5, Theorem 4.4]). Consider a positive kernel $\left\{\kappa_{n}\right\}, n \in \mathbb{N}$ in $L_{2 \pi}^{1}(\mathbb{R})$ and the corresponding sequence $\left\{L_{n}\right\}$ of positive linear operators defined by (5.3). If 
$\beta_{n}$ are defined in (5.4), then the following properties are equivalent:

1. $\lim _{n \rightarrow \infty} \beta_{n}=0$,

2. For every $1 \leq p<\infty$ and $f \in L_{2 \pi}^{p}(\mathbb{R})$

$$
\lim _{n \rightarrow \infty} L_{n}(f)=f \quad \text { in } \quad L_{2 \pi}^{p}(\mathbb{R})
$$

as well as

$$
\lim _{n \rightarrow \infty} L_{n}(f)=f \quad \text { in } \quad C_{2 \pi}(\mathbb{R})
$$

provided $f \in C_{2 \pi}(\mathbb{R})$.

Example 5.6. For the Jackson operator $\left\{\mathcal{J}_{n}\right\}$, we have

1. $\lim _{n \rightarrow \infty} \mathcal{J}_{n}(1 ; x)=1$,

2. $\lim _{n \rightarrow \infty} \mathcal{J}_{n}(\sin t ; x)=\lim _{n \rightarrow \infty}\left(\frac{2 n^{2}-2}{2 n^{2}+1} \sin x\right)=\sin x$,

3. $\lim _{n \rightarrow \infty} \mathcal{J}_{n}(\cos t ; x)=\lim _{n \rightarrow \infty}\left(\frac{2 n^{2}-2}{2 n^{2}+1} \cos x\right)=\cos x$.

Therefore, Theorem 5.3 implies that

$$
\lim _{n \rightarrow \infty} \mathcal{J}_{n}(f ; x)=f(x), \quad \text { uniformly in } \quad C_{2 \pi}(\mathbb{R})
$$

provided $f \in C_{2 \pi}(\mathbb{R})$. Also, since

$$
\beta_{n}=\frac{3}{\pi}\left(4 n^{3}+2 n\right)^{-1} \int_{-\pi}^{\pi} \frac{\left(\sin \frac{n t}{2}\right)^{4}}{\left(\sin \frac{t}{2}\right)^{2}} d t=\frac{3}{2\left(2 n^{2}+1\right)}
$$

we have that

$$
\lim _{n \rightarrow \infty} \beta_{n}=\lim _{n \rightarrow \infty} \frac{3}{2\left(2 n^{2}+1\right)}=0 .
$$

and Theorem 5.5 yields (5.6) as well. 
Example 5.7. For de la Vallée Poussin operator, we have

1. $\lim _{n \rightarrow \infty} L_{n}(1 ; x)=1$,

2. $\lim _{n \rightarrow \infty} L_{n}(\sin t ; x)=\lim _{n \rightarrow \infty}\left(\frac{n}{n+1} \sin x\right)=\sin x$,

3. $\lim _{n \rightarrow \infty} L_{n}(\cos t ; x)=\lim _{n \rightarrow \infty}\left(\frac{n}{n+1} \cos x\right)=\cos x$.

Also,

$$
\beta_{n}=\frac{(n !)^{2}}{2 \pi(2 n) !} \int_{-\pi}^{\pi}\left(\sin \frac{t}{2}\right)^{2}\left(2 \cos \left(\frac{t}{2}\right)\right)^{2 n} d t=\frac{1}{2(n+1)}
$$

Thus,

$$
\lim _{n \rightarrow \infty} \beta_{n}=\lim _{n \rightarrow \infty} \frac{1}{2(n+1)}=0
$$

By Theorem 5.5 or Theorem 5.3, we have the uniform convergence of de la Vallée Poussin operator $L_{n}(f ; x)$ to $f$.

Example 5.8. For $p \geq 2$, let $\beta_{n}$ be defined for operators from (3.4):

$$
\begin{aligned}
\beta_{n} & =\frac{1}{2 \pi c_{n, p}} \int_{-\pi}^{\pi} \sin ^{2}\left(\frac{t}{2}\right)\left(\frac{\sin \frac{n t}{2}}{\sin \frac{t}{2}}\right)^{2 p} d t \\
& =\frac{1}{2 \pi c_{n, p}} \int_{-\pi}^{\pi} \frac{\left(\sin \frac{n t}{2}\right)^{2 p}}{\left(\sin \frac{t}{2}\right)^{2 p-2}} d t \\
& =\frac{c_{n, p, p-1}}{c_{n, p, p}}
\end{aligned}
$$


where constants $c_{n, p, q}$ are defined in [42, $\left.p .14\right]$. Then,

$$
\begin{aligned}
\beta_{n}=(2 p-1)(2 p-2) \frac{\sum_{\nu=1}^{p-1}\left(\begin{array}{c}
2 p \\
p-\nu
\end{array}\right)(\nu n+p-2) \ldots(\nu n-p+2)}{\sum_{\nu=1}^{p}\left(\begin{array}{c}
2 p \\
p-\nu
\end{array}\right)(\nu n+p-1) \ldots(\nu n-p+1)} \\
=(2 p-1)(2 p-2) \frac{n^{2 p-3}\left(\left(\begin{array}{c}
2 p \\
p-1
\end{array}\right)+\cdots+\left(\begin{array}{c}
2 p \\
1
\end{array}\right)\right)+\ldots}{n^{2 p-1}\left(\left(\begin{array}{c}
2 p \\
p-1
\end{array}\right)+\cdots+\left(\begin{array}{c}
2 p \\
0
\end{array}\right)\right)+\ldots},
\end{aligned}
$$

and so

$$
\lim _{n \rightarrow \infty} \beta_{n}=0 .
$$

Therefore, Theorem 5.5 implies that

$$
\lim _{n \rightarrow \infty} L_{n, p}(f)=f \quad \text { in } \quad L_{2 \pi}^{p}(\mathbb{R})
$$

for every $1 \leq p<\infty$ and $f \in L_{2 \pi}^{p}(\mathbb{R})$, and

$$
\lim _{n \rightarrow \infty} L_{n, p}(f)=f \quad \text { in } \quad C_{2 \pi}(\mathbb{R}),
$$

if $f \in C_{2 \pi}(\mathbb{R})$.

The next theorem deals with the following question, in Theorem 5.1, is it possible to replace the three functions $1, x, x^{2}$ with any three arbitrary functions $f_{0}(x), f_{1}(x), f_{2}(x)$ ? If this is possible, what properties should these functions have? Korvkin answered this question and generalized Theorem 5.1 .

Theorem 5.9 ([37, Theorem 8, p. 49]). If the three conditions

$$
L_{n}\left(f_{i} ; x\right)=f_{i}(x)+\alpha_{i, n}(x)
$$

hold for the sequence of linear positive operators $L_{n}(f ; x)$, where $\left\{f_{i}\right\}$ is a Chebyshev 
system in the interval $[a, b]$ and $\alpha_{i, n}(x), i=0,1,2$, converge uniformly to zero in this interval, then the sequence $L_{n}(f ; x)$ converges uniformly to $f(x)$ in the interval $[a, b]$ whenever the function $f(x)$ is continuous in this interval.

It is straightforward to verify that Theorems 5.1 is a special case of this theorem when $f_{i}(x)=x^{i}, i=0,1,2$, since $\left\{1, x, x^{2}\right\}$ forms a Chebyshev system on any interval $[a, b]$.

Example 5.10. For the $\mathcal{B B H}$ operator, by using (3.11), we have

$$
\begin{aligned}
& \lim _{n \rightarrow \infty} L_{n}(1, x)=1, \\
& \lim _{n \rightarrow \infty} L_{n}\left(\frac{t}{t+1}, x\right)=\frac{x}{x+1}, \\
& \lim _{n \rightarrow \infty} L_{n}\left(\left(\frac{t}{t+1}\right)^{2}, x\right)=\left(\frac{x}{x+1}\right)^{2} .
\end{aligned}
$$

We showed that $\varphi_{j}(t)=\left(\frac{t}{t+1}\right)^{j}, j=0,1,2$, is a Chebyshev system on $[0, b]$. Thus, on any compact subset of $[0, \infty)$, we have

$$
\lim _{n \rightarrow \infty} L_{n}(f ; x)=f(x)
$$

Theorem 5.11 (Schurer Theorem, [50, Theorem 1]). Let $H\left(x_{0}\right)$ denote the class of real functions which are defined on the whole real axis and satisfy the following conditions:

1. $f$ is continuous at $x=x_{0}$,

2. $f(x)=\mathcal{O}\left(x^{2}\right)$ when $|x| \rightarrow \infty$.

Let $f \in H\left(x_{0}\right)$ and $\left\{L_{n}\right\}$ be a sequence of positive linear operators defined on $H\left(x_{0}\right)$. If for all $n \geq 1$ we have,

1. $L_{n}(1 ; x)=1+\alpha_{n}(x)$, 
2. $L_{n}(t ; x)=x+\beta_{n}(x)$,

3. $L_{n}\left(t^{2} ; x\right)=x^{2}+\gamma_{n}(x)$,

and if

$$
\lim _{n \rightarrow \infty} \alpha_{n}(x)=\lim _{n \rightarrow \infty} \beta_{n}(x)=\lim _{n \rightarrow \infty} \gamma_{n}(x)=0,
$$

then

$$
\lim _{n \rightarrow \infty} L_{n}\left(f ; x_{0}\right)=f\left(x_{0}\right) .
$$

In some sense, Schurer's Theorem is a generalization of Korovkin's Theorem, since the space of functions has been extended.

However, note that the uniform convergence in Theorem 5.1 has been replaced by the pointwise convergence in Theorem 5.11 .

\subsection{A Korovkin-type Theorem for Unbounded Func- tions with Non-compact Support}

One "shortcoming" of the Bohman-Korovkin Theorem is the fact that it is not applicable for unbounded functions with non-compact support. For example, consider the sequence of positive linear operators $\left\{L_{n}\right\}$ which is defined as

$$
L_{n}(f ; x)=B_{n}(f ; x)+f(n) e^{-n},
$$

where $B_{n}(f ; x)$ is the Bernstein operator. It is easy to check that $L_{n}(f ; x)$ is positive linear operator:

$$
\begin{aligned}
L_{n}(\alpha f+\beta g ; x) & =B_{n}(\alpha f+\beta g ; x)+(\alpha f(n)+\beta g(n)) e^{-n} \\
& =\alpha\left(B_{n}(f ; x)+f(n) e^{-n}\right)+\beta\left(B_{n}(g ; x)+g(n) e^{-n}\right) \\
& =\alpha L_{n}(f ; x)+\beta L_{n}(g ; x) .
\end{aligned}
$$


For $k=0,1,2$, we now have

$$
\begin{aligned}
\lim _{n \rightarrow \infty} L_{n}\left(t^{k} ; x\right) & =\lim _{n \rightarrow \infty}\left(B_{n}\left(t^{k} ; x\right)+n^{k} e^{-n}\right) \\
& =\lim _{n \rightarrow \infty} B_{n}\left(t^{k} ; x\right)+\lim _{n \rightarrow \infty}\left(n^{k} e^{-n}\right) \\
& =x^{k} .
\end{aligned}
$$

However, for the function $f(x)=e^{x}$, we have

$$
\lim _{n \rightarrow \infty} L_{n}\left(e^{t} ; x\right)=\lim _{n \rightarrow \infty}\left(B_{n}\left(e^{t} ; x\right)+e^{n} e^{-n}\right)=e^{x}+1 .
$$

So, $L_{n}(f ; x) \nrightarrow f$ as $n \rightarrow \infty$.

The following result was proved by Boyanov and Veselinov in 1970.

Theorem 5.12 (Boyanov-Veselinov Theorem, [12, Theorem 2]). Let $C^{*}[0, \infty)$ be the space of continuous real-valued functions on $[0, \infty)$ such that $\lim _{x \rightarrow \infty} f(x)$ exists, and let $\left\{L_{n}\right\}$ be a sequence of positive linear operators,

$$
L_{n}: C^{*}[0, \infty) \rightarrow C^{*}[0, \infty)
$$

Then,

$$
\lim _{n \rightarrow \infty} L_{n}(f ; x)=f(x) \quad \text { uniformly in }[0, \infty) \text {, }
$$

for any $f \in C^{*}[0, \infty)$, provided that

$$
\lim _{n \rightarrow \infty} L_{n}\left(e^{-k t} ; x\right)=e^{-k t} \quad \text { uniformly in }[0, \infty), \quad \text { for } k=0,1,2 \text {. }
$$

The next examples illustrate convergence of the Favard-Szász-Mirakjan operator and the Baskakov operator by using Theorem 5.12 . 
Example 5.13. It is easy to check that $\mathcal{F}_{n}$ satisfies conditions (5.9). We have,

$$
\begin{aligned}
& \lim _{n \rightarrow \infty} \mathcal{F}_{n}(1 ; x)=\lim _{n \rightarrow \infty} \sum_{k=0}^{\infty} e^{-n x} \frac{(n x)^{k}}{k !}=1, \\
& \lim _{n \rightarrow \infty} \mathcal{F}_{n}\left(e^{-t} ; x\right)=\lim _{n \rightarrow \infty} \sum_{k=0}^{\infty} e^{-n x} \frac{(n x)^{k}}{k !} e^{\frac{-k}{n}}=e^{-x}, \\
& \lim _{n \rightarrow \infty} \mathcal{F}_{n}\left(e^{-2 t} ; x\right)=\lim _{n \rightarrow \infty} \sum_{k=0}^{\infty} e^{-n x} \frac{(n x)^{k}}{k !} e^{\frac{-2 k}{n}}=e^{-2 x} .
\end{aligned}
$$

Since the conditions of the Boyanov-Veselinov Theorem are satisfied, we have

$$
\lim _{n \rightarrow \infty} \mathcal{F}_{n}(f ; x)=f(x)
$$

Example 5.14. The Baskakov operator satisfies conditions (5.9) since

$$
\begin{aligned}
\lim _{n \rightarrow \infty} L_{n}(1 ; x)= & \lim _{n \rightarrow \infty} \sum_{k=0}^{\infty}\left(\begin{array}{c}
n+k-1 \\
k
\end{array}\right) \frac{x^{k}}{(1+x)^{n+k}}=1, \\
\lim _{n \rightarrow \infty} L_{n}\left(e^{-t} ; x\right) & =\lim _{n \rightarrow \infty} \sum_{k=0}^{\infty}\left(\begin{array}{c}
n+k-1 \\
k
\end{array}\right) \frac{x^{k}}{(1+x)^{n+k}} e^{-\frac{k}{n}} \\
& =\lim _{n \rightarrow \infty}\left(1+x-e^{-\frac{1}{n}} x\right)^{-n} \\
& =e^{-x}
\end{aligned}
$$

and

$$
\begin{aligned}
\lim _{n \rightarrow \infty} L_{n}\left(e^{-2 t} ; x\right) & =\lim _{n \rightarrow \infty} \sum_{k=0}^{\infty}\left(\begin{array}{c}
n+k-1 \\
k
\end{array}\right) \frac{x^{k}}{(1+x)^{n+k}} e^{-2 \frac{k}{n}} \\
& =\lim _{n \rightarrow \infty}\left(1+x-e^{-\frac{2}{n}} x\right)^{-n} \\
& =e^{-2 x}
\end{aligned}
$$

Therefore, by the Boyanov-Veselinov Theorem we have

$$
\lim _{n \rightarrow \infty} L_{n}(f ; x)=f(x) .
$$




\subsection{Shisha-Mond Quantitative Estimates}

In 1968, Shisha ${ }^{2}$ and Mond presented a quantitative form of Bohman-Korovkin's Theorem in order to measure the speed of convergence. Let $[a, b]$ be an interval such that $-\infty<a<b<\infty$ and let $\|\cdot\|$ denote the uniform norm over $[a, b]$.

Theorem 5.15 ([51, Theorem 1]). Let $f \in C[a, b]$ and $\left\{L_{n}\right\}$ be a sequence of positive linear operators on $[a, b]$ such that, for each $n, L_{n}(1 ; x)$ is bounded. Then,

$$
\left\|L_{n}(f)-f\right\| \leq\|f\|\left\|L_{n}(1)-1\right\|+\left\|L_{n}(1)+1\right\| \omega_{1}\left(f, \mu_{n}\right),
$$

where

$$
\mu_{n}:=\left\|L_{n}\left((t-x)^{2} ; x\right)\right\|^{\frac{1}{2}} .
$$

For trigonometric operators, we can define

$$
\mu_{n}:=\left\|L_{n}\left(2 \sin ^{2} \frac{(t-x)^{2}}{2} ; x\right)\right\|^{\frac{1}{2}}
$$

for $f \in C[0,2 \pi]$.

Moreover, these results were also generalized by Shisha and Mond in their paper. Let $f_{i} \in C[a, b], i=0,1,2$, be such that $\left\{f_{0}, f_{1}, f_{2}\right\}$ is a Chebyshev system. Define

$$
F(t, x)=\left|\begin{array}{ccc}
f_{0}(x) & f_{1}(x) & f_{2}(x) \\
f_{0}^{\prime}(x) & f_{1}^{\prime}(x) & f_{2}^{\prime}(x) \\
f_{0}(t) & f_{1}(t) & f_{2}(t)
\end{array}\right|,
$$

\footnotetext{
${ }^{2}$ Oved Shisha (1932-1995). His main research contributions are in real, complex, and numerical analysis, and in particular, approximation theory, integration, and inequalities. In 1968, he began publication of the Journal of Approximation Theory. He was Editor-in-Chief of the Journal until 1990, when he was named Founding Editor. http://www.math.technion.ac.il/hat/people/ obits/shisha.pdf
} 
and note that either for any $t, x \in[a, b]$ we have $F(x, x)=0$ and $F(t, x) \geq 0$ or $-F$ has these conditions. For example, if $f_{0}=1, f_{1}=x$ and $f_{2}=x^{2}$, then

$$
F(t, x)=\left|\begin{array}{lll}
f_{0}(x) & f_{1}(x) & f_{2}(x) \\
f_{0}^{\prime}(x) & f_{1}^{\prime}(x) & f_{2}^{\prime}(x) \\
f_{0}(t) & f_{1}(t) & f_{2}(t)
\end{array}\right|=\left|\begin{array}{ccc}
1 & x & x^{2} \\
0 & 1 & 2 x \\
1 & t & t^{2}
\end{array}\right|=(t-x)^{2}
$$

Thus, $F(x, x)=0$ and $F(t, x) \geq 0$.

Similarly, if $f_{0}=1, f_{1}=\sin x$ and $f_{2}=\cos x$, then

$$
F(t, x)=\left|\begin{array}{lll}
f_{0}(x) & f_{1}(x) & f_{2}(x) \\
f_{0}^{\prime}(x) & f_{1}^{\prime}(x) & f_{2}^{\prime}(x) \\
f_{0}(t) & f_{1}(t) & f_{2}(t)
\end{array}\right|=\left|\begin{array}{ccc}
1 & \sin x & \cos x \\
0 & \cos x & -\sin x \\
1 & \sin t & \cos t
\end{array}\right|=2 \sin ^{2} \frac{(t-x)}{2}
$$

Shisha and Mond [51, Theorem 2] demonstrated that Theorem 5.15 can be generalized as follows.

Theorem 5.16 ([51, Theorem 2]). Let $\alpha_{i}$ and $f_{i}, i=0,1,2$, be real functions, defined and bounded in $[a, b]$. Assume that for any $t, x \in[a, b]$ we have

$$
F(t, x):=\sum_{k=0}^{2} \alpha_{k}(x) f_{k}(t) \geq K(t-x)^{2}, \quad F(x, x)=0,
$$

where $K$ is a positive constant depending on neither $x$ nor $t$. Suppose that $f \in C[a, b]$ and the sequence of operators $\left\{L_{n}\right\}$ satisfies the conditions of Theorem 5.15. Then,

$$
\left\|L_{n}(f)-f\right\| \leq\|f\|\left\|L_{n}(1)-1\right\|+\left\|L_{n}(1)+1\right\| \omega_{1}\left(f, \mu_{n}\right),
$$

where

$$
\mu_{n}:=\left\|L_{n}(F(t ; x) ; x) / K\right\|^{\frac{1}{2}}
$$


In the trigonometric case, $\mu_{n}$ can be estimated as follows

$$
\begin{aligned}
& \left\|L_{n}\left(\sin ^{2} \frac{(t-x)}{2} ; x\right)\right\|=\left\|L_{n}\left(\frac{1}{2}-\frac{\cos (t-x)}{2} ; x\right)\right\| \\
& =\frac{1}{2}\left\|L_{n}(1-\cos t \cos x-\sin t \sin x ; x)\right\| \\
& =\frac{1}{2}\left\|L_{n}(1 ; x)-\cos x L_{n}(\cos t ; x)-\sin x L_{n}(\sin t ; x)\right\| \\
& =\frac{1}{2}\left\|L_{n}(1 ; x)-\cos x L_{n}(\cos t ; x)-\sin x L_{n}(\sin t ; x)+\sin ^{2} x+\cos ^{2} x-1\right\| \\
& \leq \frac{1}{2}\left\|L_{n}(1 ; x)-1\right\|+\frac{1}{2}\|\cos x\|\left\|\cos x-L_{n}(\cos t ; x)\right\| \\
& +\frac{1}{2}\|\sin x\|\left\|\sin x-L_{n}(\sin t ; x)\right\| .
\end{aligned}
$$

Using the above results, one can show that, for the Jackson operator, we have

$$
\left\|\mathcal{J}_{n}(f)-f\right\| \leq C \omega_{1}\left(f, \frac{1}{n}\right)
$$

where $C$ is a constant (independent of $f$ ). For the de la Vallée Poussin operator , we obtain

$$
\left\|L_{n}(f)-f\right\| \leq C \omega_{1}\left(f, \frac{1}{\sqrt{n}}\right),
$$

which can be found in Natanson ${ }^{3}$ [44, pp. 183-184].

\subsection{Voronovskaja's Formula}

Let $I \subset \mathbb{R}$ be an interval and $\left\{L_{n}\right\}_{n \geqslant 1}$ be sequence of positive linear operators, where the operators $L_{n}$ 's are defined on $C(I)$. In order to study the asymptotic behavior

\footnotetext{
${ }^{3}$ Isidor Pavlovich Natanson (1906 - 1964) graduated from Leningrad University in 1929. He made his first research concerning singular integrals. In the 30s, S.N.Bernstein worked at the Leningrad University. Under his influence, Natanson began to work in the constructive theory of functions. His research concerned series in orthogonal polynomials, interpolation processes, the moment problem, Gibbs phenomenon and other problems of the approximation theory. He also worked in the "pure" theory of functions and functional analysis. http://analysis.spbu.ru/ history/natans_i/index-e.html
} 
of these operators, we need to examine

$$
\lim _{n \rightarrow \infty} n\left(L_{n}(f ; x)-f(x)\right)
$$

thus obtaining the Voronovskaja formula for the sequence of $\left\{L_{n}\right\}_{n \geqslant 1}$. For $r \in \mathbb{N}$, an asymptotic expansion for $\left\{L_{n}\right\}$ of order $r$ is

$$
L_{n}(f ; x)=f(x)+\sum_{k=1}^{r} \frac{\alpha_{k}(f, x)}{n^{k}}+o\left(n^{-r}\right) .
$$

Thus, Voronovskaja's formula for a sequence of positive linear operators

$$
\lim _{n \rightarrow \infty} n\left(L_{n}(f ; x)-f(x)\right)=\alpha_{1}(f, x)
$$

is actually based on an asymptotic expansion of order 1 ,

$$
L_{n}(f ; x)=f(x)+\frac{\alpha_{1}(f, x)}{n}+o\left(n^{-1}\right)
$$

In 1932, in her paper "Détermination de la forme asymptotique de l'approximation des fonctions par les polynômes de M. Bernstein", Voronovskaja proved the following theorem for the Bernstein operator .

Theorem 5.17 (see e.g. [38, p. 22]). Let $f$ be bounded in $[0,1]$ and suppose that the second derivative $f^{\prime \prime}(x)$ exists at the certain point $x$ of $[0,1]$. Then,

$$
\lim _{n \rightarrow \infty} n\left(B_{n}(f ; x)-f(x)\right)=\frac{x(1-x)}{2} f^{\prime \prime}(x) .
$$

Therefore, she proved that,

$$
B_{n}(f ; x)=f(x)+\frac{x(1-x)}{2 n} f^{\prime \prime}(x)+o\left(n^{-1}\right) .
$$


Voronovskaja's results says that if $f^{\prime \prime}(x) \neq 0$, the rate of convergence of $B_{n}(f ; x)$ to $f(x)$ cannot be better than of order $1 / n$ as $n \rightarrow \infty$.

For a continuous function $f \in C[0,1]$ which is twice differentiable at the point $x \in(0,1)$ and for positive linear operators $L_{n}: C[0,1] \rightarrow C[0,1]$ which preserve constants, Cárdenas-Morales, Garrancho, and Raşa proved the following analogue of Voronovskaja's theorem.

Theorem 5.18 ([14, Theorem 2.1]). Let $\left\{\lambda_{n}\right\}$ be a sequence of positive numbers such that $\lambda_{n} \rightarrow \infty$ as $n \rightarrow \infty$. Suppose that the function $\varphi(x)$ satisfies the following conditions:

1. $\varphi$ is infinity differentiable on $[0,1]$,

2. $\varphi^{\prime}(x)>0, x \in(0,1)$,

3. $\varphi(0)=0$ and $\varphi(1)=1$.

Then, we have

$$
\lim _{n \rightarrow \infty} \lambda_{n}\left(L_{n}(f ; x)-f(x)\right)=p(x) f^{\prime \prime}(x)+q(x) f^{\prime}(x),
$$

provided that

$$
\begin{aligned}
& \lim _{n \rightarrow \infty} \lambda_{n} L_{n}(\varphi(t)-\varphi(x) ; x)=p(x) \varphi^{\prime \prime}(x)+q(x) \varphi^{\prime}(x), \\
& \lim _{n \rightarrow \infty} \lambda_{n} L_{n}\left((\varphi(t)-\varphi(x))^{2} ; x\right)=2 p(x) \varphi^{\prime}(x)^{2}, \\
& \lim _{n \rightarrow \infty} \lambda_{n} L_{n}\left((\varphi(t)-\varphi(x))^{4} ; x\right)=0,
\end{aligned}
$$

where $p, q$ are two continuous functions defined on $[0,1]$ such that $p$ is strictly positive on $(0,1)$.

We illustrate applications of this theorem for a few operators defined previously. 
Example 5.19. The modified Durrmeyer operator $U_{n}$ defined in (4.7) has the following properties:

1. $U_{n}(t-x ; x)=0$,

2. $\lim _{n \rightarrow \infty} n U_{n}\left((t-x)^{2} ; x\right)=2 x(1-x)$,

3. $\lim _{n \rightarrow \infty} n U_{n}\left((t-x)^{4} ; x\right)=0$.

Hence, the conditions of Theorem 5.18 are satisfied with $\lambda_{n}=n, \varphi(x)=x, p(x)=$ $x(1-x)$ and $q(x)=0$. Therefore,

$$
\lim _{n \rightarrow \infty} n\left(U_{n}(f ; x)-f(x)\right)=x(1-x) f^{\prime \prime}(x)
$$

In the next example, we will deal with the Durrmeyer operator. Derriennic [20, Theorem II.5, p. 329] demonstrated that if $f$ is integrable, bounded on [0,1] and admits a second derivative at the point $x \in[0,1]$, then

$$
\lim _{n \rightarrow \infty} n\left(\mathcal{D}_{n}(f ; x)-f(x)\right)=(1-2 x) f^{\prime}(x)+x(1-x) f^{\prime \prime}(x) .
$$

Moreover, this limit is uniform if $f^{\prime \prime}$ is continuous on $[0,1]$. The idea of the proof was based on

$$
\mathcal{D}_{n}(f ; x)=f(x)+f^{\prime}(x)\left(\frac{1-2 x}{n+2}\right)+f^{\prime \prime}(x)\left(\frac{(n-3) x(1-x)+1}{(n+2)(n+3)}\right)+E(n, x),
$$

with $E(n, x)$ such that

$$
|n E(n, x)| \leq K \omega\left(f^{\prime \prime}, \frac{1}{\sqrt{n}}\right) .
$$

Example 5.20. Let $\lambda_{n}=n$ and $\varphi(x)=x$, we now check the conditions of Theorem 
5.18 to demonstrate (5.15) in another way. First, we have

$$
\begin{aligned}
\lim _{n \rightarrow \infty} n \mathcal{D}_{n}(t-x ; x) & =\lim _{n \rightarrow \infty} n\left(\mathcal{D}_{n}(t ; x)-x \mathcal{D}_{n}(1 ; x)\right) \\
& =\lim _{n \rightarrow \infty} n\left(\frac{1-2 x}{n+2}\right) \\
& =1-2 x .
\end{aligned}
$$

We conclude that $q(x)=1-2 x$, since $\varphi^{\prime}(x)=1$ and $\varphi^{\prime \prime}(x)=0$. Now,

$$
\begin{aligned}
\lim _{n \rightarrow \infty} n \mathcal{D}_{n}\left((t-x)^{2} ; x\right) & =\lim _{n \rightarrow \infty} n\left(\mathcal{D}_{n}\left(t^{2} ; x\right)-2 x \mathcal{D}_{n}(t ; x)+x^{2} \mathcal{D}_{n}(1 ; x)\right) \\
& =\lim _{n \rightarrow \infty}\left(n^{2} \frac{2 x(1-x)}{(n+2)(n+3)}+\frac{n\left(6 x^{2}-6 x+2\right)}{(n+2)(n+3)}\right) \\
& =2 x(1-x),
\end{aligned}
$$

which implies that $p(x)=x(1-x)$. It remains to prove that

$$
\lim _{n \rightarrow \infty} n \mathcal{D}_{n}\left((t-x)^{4} ; x\right)=0 .
$$

It is clear that

$$
\begin{aligned}
& \lim _{n \rightarrow \infty} n \mathcal{D}_{n}\left((t-x)^{4} ; x\right) \\
& =\lim _{n \rightarrow \infty} n\left(\mathcal{D}_{n}\left(t^{4} ; x\right)-4 x \mathcal{D}_{n}\left(t^{3} ; x\right)+6 x^{2} \mathcal{D}_{n}\left(t^{2} ; x\right)-4 x^{3} \mathcal{D}_{n}(t ; x)+x^{4} \mathcal{D}_{n}(1 ; x)\right) .
\end{aligned}
$$

Using the following formula, that can be found in Derriennic's paper [20, p. 326],

$$
\mathcal{D}_{n}\left(t^{m} ; x\right)=\frac{(n+1) !}{(n+m+1) !} \sum_{k=0}^{m}\left(\begin{array}{l}
m \\
k
\end{array}\right)\left(\begin{array}{l}
n \\
k
\end{array}\right) m ! x^{k} .
$$


we have

$$
\begin{aligned}
& \lim _{n \rightarrow \infty} n \mathcal{D}_{n}\left((t-x)^{4} ; x\right) \\
= & \lim _{n \rightarrow \infty} n\left(4 ! \frac{1+\left(\begin{array}{l}
4 \\
1
\end{array}\right)\left(\begin{array}{l}
n \\
1
\end{array}\right) x+\left(\begin{array}{l}
4 \\
2
\end{array}\right)\left(\begin{array}{l}
n \\
2
\end{array}\right) x^{2}+\left(\begin{array}{l}
4 \\
3
\end{array}\right)\left(\begin{array}{l}
n \\
3
\end{array}\right) x^{3}+\left(\begin{array}{l}
4 \\
4
\end{array}\right)\left(\begin{array}{l}
n \\
4
\end{array}\right) x^{4}}{(n+2)(n+3)(n+4)(n+5)}\right) \\
& +\lim _{n \rightarrow \infty} n\left(3 !(-4 x) \frac{1+\left(\begin{array}{l}
3 \\
1
\end{array}\right)\left(\begin{array}{l}
n \\
1
\end{array}\right) x+\left(\begin{array}{l}
3 \\
2
\end{array}\right)\left(\begin{array}{l}
n \\
2
\end{array}\right) x^{2}+\left(\begin{array}{l}
3 \\
3
\end{array}\right)\left(\begin{array}{l}
n \\
3
\end{array}\right) x^{3}}{(n+2)(n+3)(n+4)}\right) \\
& +\lim _{n \rightarrow \infty} n\left(2 !\left(6 x^{2}\right) \frac{1+\left(\begin{array}{l}
2 \\
1
\end{array}\right)\left(\begin{array}{l}
n \\
1
\end{array}\right) x+\left(\begin{array}{l}
2 \\
2
\end{array}\right)\left(\begin{array}{l}
n \\
2
\end{array}\right) x^{2}}{(n+2)(n+3)}\right) \\
& +\lim _{n \rightarrow \infty} n\left(-4 x^{3} \frac{1+n x}{n+2}+x^{4}\right)=0 .
\end{aligned}
$$

Thus, we have

$$
\begin{aligned}
\lim _{n \rightarrow \infty} n\left(\mathcal{D}_{n}(f ; x)-f(x)\right) & =p(x) f^{\prime \prime}(x)+q(x) f^{\prime}(x) \\
& =x(1-x) f^{\prime \prime}(x)+(1-2 x) f^{\prime}(x),
\end{aligned}
$$

which is (5.15).

For the Bleimann-Butzer-Hahn operator defined in 3.10$)$, we have the following theorem that was proved by Abel and Ivan [1, Theorem 4.4].

Theorem 5.21 ([1], Theorem 4.4]). If $f$ is a real-valued function defined on $[0, \infty)$, if it is twice differentiable in some neighborhood of a point $x \in[0, \infty)$, and if $f(x)=$ $\mathcal{O}(1+x)$, then

$$
\lim _{n \rightarrow \infty} n\left(L_{n}(f ; x)-f(x)\right)=\frac{x(1+x)^{2}}{2} f^{\prime \prime}(x) .
$$

We will use Theorem 5.18 to prove 5.17 but with a restriction to $[0,1]$. Suppose that $\varphi(x):=\frac{2 x}{x+1}$ in Theorem 5.18 which implies, in particular, that that $\varphi(0)=0$ 
and $\varphi(1)=1$. We have

$$
\begin{aligned}
\lim _{n \rightarrow \infty} n L_{n}\left(\frac{2 t}{t+1}-\frac{2 x}{x+1} ; x\right) & =\frac{-2 x}{x+1} \\
& =p(x) \frac{-4}{(x+1)^{3}}+q(x) \frac{2}{(x+1)^{2}}
\end{aligned}
$$

and

$$
\begin{aligned}
\lim _{n \rightarrow \infty} n L_{n}\left(\left(\frac{2 t}{t+1}-\frac{2 x}{x+1}\right)^{2} ; x\right) & =-4\left(\frac{x}{x+1}\right)^{2}+4\left(\frac{x}{x+1}\right) \\
& =2 p(x) \frac{4}{(x+1)^{4}}
\end{aligned}
$$

Hence, $p(x)=\frac{x(1+x)^{2}}{2}$ and $q(x)=0$. It is also possible to show that

$$
\lim _{n \rightarrow \infty} n L_{n}\left(\left(\frac{2 t}{t+1}-\frac{2 x}{x+1}\right)^{4} ; x\right)=0
$$

Hence, Theorem 5.18 implies

$$
\lim _{n \rightarrow \infty} n\left(L_{n}(f ; x)-f(x)\right)=\frac{x(1+x)^{2}}{2} f^{\prime \prime}(x) .
$$

In 1959, Mamedov proved the following theorem that is a special case of Theorem 5.18

Theorem 5.22 (see, for example, [55, Theorem B]). Let $\left\{L_{n}\right\}$ be a sequence of positive linear operators such that, for all $x \in[a, b]$, we have

1. $L_{n}(1 ; x)=1$,

2. $L_{n}(t ; x)=x$,

3. $L_{n}\left(t^{2} ; x\right)=x^{2}+\frac{\psi(x)}{n}+o\left(\frac{1}{n}\right)$,

4. $L_{n}\left((t-x)^{4} ; x\right)=o\left(\frac{1}{n}\right)$. 
Then, for each $f \in C^{2}[a, b]$, we have

$$
L_{n}(f ; x)-f(x)=\frac{\psi(x) f^{\prime \prime}(x)}{2 n}+o\left(\frac{1}{n}\right), \quad x \in[a, b] .
$$

Note that (5.18) implies

$$
\lim _{n \rightarrow \infty} n\left(L_{n}(f ; x)-f(x)\right)=\frac{\psi(x) f^{\prime \prime}(x)}{2}, \quad x \in[a, b] .
$$

To see that Theorem 5.22 is a special case of Theorem 5.18, we let $\varphi(x)=x$ and $\lambda_{n}=n$ and check the conditions in (5.14). Since

$$
\lim _{n \rightarrow \infty} \lambda_{n} L_{n}(\varphi(t)-\varphi(x) ; x)=\lim _{n \rightarrow \infty} n L_{n}(t-x ; x)=0,
$$

we have $q(x)=0$. Then,

$$
\lim _{n \rightarrow \infty} \lambda_{n} L_{n}\left((\varphi(t)-\varphi(x))^{2} ; x\right)=\lim _{n \rightarrow \infty} n L_{n}\left((t-x)^{2} ; x\right)=\psi(x),
$$

which yields $p(x)=\psi(x) / 2$. Finally,

$$
\lim _{n \rightarrow \infty} \lambda_{n} L_{n}\left((\varphi(t)-\varphi(x))^{4} ; x\right)=\lim _{n \rightarrow \infty} n L_{n}\left((t-x)^{4} ; x\right)=0,
$$

and so 5.13$)$ implies

$$
\lim _{n \rightarrow \infty} n\left(L_{n}(f ; x)-f(x)\right)=\frac{\psi(x) f^{\prime \prime}(x)}{2} .
$$

As an example, for the Bernstein operator we have,

1. $B_{n}(1 ; x)=1$,

2. $B_{n}(t ; x)=x$, 
3. $B_{n}\left(t^{2} ; x\right)=x^{2}+\frac{x-x^{2}}{n}$,

4. $B_{n}\left((t-x)^{4} ; x\right)=\frac{1}{n^{2}}\left(3 x^{4}-6 x^{3}+7 x^{2}\right)+\frac{1}{n^{3}}\left(-6 x^{4}+12 x^{3}-7 x^{2}+x\right)=o\left(\frac{1}{n}\right)$.

Then, for each $f \in C^{2}[a, b]$, we have

$$
B_{n}(f ; x)-f(x)=\frac{x-x^{2}}{2 n} f^{\prime \prime}(x)+o\left(\frac{1}{n}\right), \quad x \in[0,1] .
$$

We now apply Theorem 5.22 to the Meyer-König-Zeller operator defined in 1.2 .

Example 5.23. In [55, Lemma 3], it was shown that

$$
M_{n}\left((t-x)^{4} ; x\right)=o\left(\frac{1}{n}\right)
$$

In [39, p. 20], it was proved that

$$
M_{n}(1 ; x)=1, \quad M_{n}(t ; x)=x .
$$

and

$$
M_{n}\left(t^{2} ; x\right)=x^{2}+\frac{x(1-x)^{2}}{n}+o\left(\frac{1}{n}\right) .
$$

Thus, from (5.19), we have

$$
\lim _{n \rightarrow \infty} n\left(M_{n}(f ; x)-f(x)\right)=\frac{x(1-x)^{2}}{2} f^{\prime \prime}(x) .
$$

We can also apply Theorem 5.18 to the Bernstein-Chlodovsky operator $(3.6)$ and obtain the result of Albrycht and Radecki [4] who showed that, if the condition

$$
\lim _{n \rightarrow \infty}\left(\frac{n}{b_{n}}\right) \exp \left(-\alpha \frac{n}{b_{n}}\right)\left(\sup _{x \in\left[0, b_{n}\right]}|f(x)|\right)=0, \quad \text { for all } \quad \alpha>0
$$


holds, then

$$
\lim _{n \rightarrow \infty}\left(\frac{n}{b_{n}}\right)\left[C_{n}(f ; x)-f(x)\right]=\frac{x}{2} f^{\prime \prime}(x) .
$$

Example 5.24. We first note that

$$
\lim _{n \rightarrow \infty} \frac{n}{b_{n}} C_{n}\left((t-x)^{4} ; x\right)=0 .
$$

Indeed, it is not difficult to show that

$$
\begin{aligned}
C_{n}\left((t-x)^{4} ; x\right)= & x^{4}\left(1-\frac{6}{n}+\frac{11}{n^{2}}-\frac{6}{n^{3}}-4+\frac{12}{n}-\frac{8}{n^{2}}+6-\frac{6}{n}-4+1\right) \\
& +x^{3}\left(\frac{6 b_{n}}{n}-\frac{18 b_{n}}{n^{2}}+\frac{12 b_{n}}{n^{3}}-\frac{12 b_{n}}{n}+\frac{12 b_{n}}{n^{2}}+\frac{6 b_{n}}{n}\right) \\
& +x^{2}\left(\frac{7 b_{n}^{2}}{n^{2}}-\frac{7 b_{n}^{2}}{n^{3}}\right)+x \frac{b_{n}^{3}}{n^{3}},
\end{aligned}
$$

and so

$$
\begin{aligned}
\lim _{n \rightarrow \infty} \frac{n}{b_{n}} C_{n}\left((t-x)^{4} ; x\right)= & x \lim _{n \rightarrow \infty}\left(\frac{b_{n}}{n}\right)^{2}+x^{2} \lim _{n \rightarrow \infty}\left(\frac{7 b_{n}}{n}-\frac{7 b_{n}}{n^{2}}\right) \\
& +x^{3} \lim _{n \rightarrow \infty}\left(-\frac{6}{n}+\frac{12}{n^{2}}\right)+x^{4} \lim _{n \rightarrow \infty}\left(\frac{3}{n b_{n}}-\frac{6}{n^{2} b_{n}}\right) \\
& =0 .
\end{aligned}
$$

Now, let $\lambda_{n}=\frac{n}{b_{n}}$ and $\varphi(x)=x$. It is easily to check that

$$
\lim _{n \rightarrow \infty} \frac{n}{b_{n}} C_{n}(t-x ; x)=0
$$

and

$$
\lim _{n \rightarrow \infty} \frac{n}{b_{n}} C_{n}\left((t-x)^{2} ; x\right)=\lim _{n \rightarrow \infty} \frac{n}{b_{n}}\left(\frac{x\left(b_{n}-x\right)}{n}\right)=x .
$$


Since $\varphi^{\prime}(x)=1$ and $\varphi^{\prime \prime}(x)=0$, it implies that

$$
q(x)=0, \quad \text { and } \quad p(x)=\frac{x}{2} .
$$

Hence, Theorem 5.18 implies that

$$
\lim _{n \rightarrow \infty} \frac{n}{b_{n}}\left(C_{n}(f ; x)-f(x)\right)=\frac{x}{2} f^{\prime \prime}(x) .
$$


6

\section{Saturation of Positive Linear}

\section{Operators}

This chapter is devoted to phenomenon of saturation; saturation in $C_{2 \pi}$ and saturation in $C[a, b]$. Karel de Leeuw [19, p. 89] described saturation as follows:

"For most methods of approximation, there is some relationship between the smoothness of the function being approximated and the rate of convergence of the approximating functions. Furthermore, there is in many cases a point beyond which increasing the smoothness of the function will not improve the degree of approximation. This is the phenomenon of saturation".

\subsection{Saturation in $C_{2 \pi}$}

The sequence $\left\{L_{n}\right\}$ of positive linear operators on $C_{2 \pi}$, is said to be saturated (see [21, pp. 48-49]) if there is a sequence of positive numbers $\{\varphi(n)\}$ which tending to 0 as $n \rightarrow \infty$ satisfying the following properties, 
1. If $f \in C_{2 \pi}$, then

$$
\liminf _{n \rightarrow \infty} \frac{\left\|f-L_{n}(f ; x)\right\|}{\varphi(n)}=0 \text { if and only if } f \text { is constant. }
$$

2. There is a non-constant function $f_{0} \in C_{2 \pi}$ such that

$$
\left\|f_{0}-L_{n}\left(f_{0} ; x\right)\right\|=\mathcal{O}(\varphi(n))
$$

The sequence $(\varphi(n))$ is called the saturation order of $\left\{L_{n}\right\}$. The saturation class of the operator $L_{n}, S\left(L_{n}\right)$, is the class of all functions $f_{0} \in C_{2 \pi}$ which satisfy 6.2 .

\subsection{Inverse and Direct Theorems}

Recall the definition of convolution operators from [21, p. 48]. Let $C_{2 \pi}=C[-\pi, \pi]$ denote the space of $2 \pi$-periodic and continuous functions and $\left\{L_{n}\right\}$ be a sequence of positive linear operators on $C_{2 \pi}$ given by the convolution formula

$$
L_{n}(f ; x):=\frac{1}{\pi} \int_{-\pi}^{\pi} f(x+t) \mathrm{d} \mu_{n}(t)
$$

where $\mathrm{d} \mu_{n}$ has the following properties:

1. $\mathrm{d} \mu_{n}$ is non-negative, even, Borel measure on $[-\pi, \pi)$,

2. $\frac{1}{\pi} \int_{-\pi}^{\pi} \mathrm{d} \mu_{n}(t)=1$.

We also recall some definitions. If

$$
f \sim \sum_{k=0}^{\infty} A_{k}(f, x)=\frac{a_{0}(f)}{2}+\sum_{k=1}^{\infty}\left(a_{k}(f) \cos k x+b_{k}(f) \sin k x\right)
$$


is the Fourier series of a function $f \in C_{2 \pi}$, where

$$
a_{k}(f):=\frac{1}{\pi} \int_{-\pi}^{\pi} f(\tau) \cos k \tau d \tau
$$

and

$$
b_{k}(f):=\frac{1}{\pi} \int_{-\pi}^{\pi} f(\tau) \sin k \tau d \tau
$$

then, $\tilde{f}$ denotes the conjugate of $f$, is defined as

$$
\tilde{f} \sim \sum_{k=1}^{\infty}\left(-b_{k}(f) \cos k x+a_{k}(f) \sin k x\right)
$$

The class of functions $f$ which satisfy

$$
g \sim \sum_{k=0}^{\infty} k^{\alpha} A_{k}(f, x) \in L_{\infty} \quad \text { and } \quad\|g\|_{\infty} \leq M
$$

is

$$
\begin{aligned}
& \widetilde{W}^{(\alpha-1)}(1, M):=\left\{f: \widetilde{f^{(\alpha-1)}} \in \operatorname{Lip}(1, M)\right\}, \quad \text { when } \alpha \text { is odd, } \\
& W^{(\alpha-1)}(1, M):=\left\{f: f^{(\alpha-1)} \in \operatorname{Lip}(1, M)\right\}, \quad \text { when } \alpha \text { is even. }
\end{aligned}
$$

There are two kinds of theorems for saturation, inverse theorems and direct theorems. A theorem is called direct (inverse) if it gives sufficient (necessary) conditions for the function $f$ to be in a saturation class. For example, an inverse theorem would be "if $f \in S\left(L_{n}\right)$, then $\sum_{k=1}^{\infty} \psi_{k} A_{k}(f, x) \in L_{\infty}$ ", and a direct theorem is "if $\sum_{k=1}^{\infty} \psi_{k} A_{k}(f, x) \in L_{\infty}$, then $f \in S\left(L_{n}\right) . "$

\subsubsection{The Tureckir Theorem and its extension}

Let $\rho_{k, n}$ be coefficients of the Fourier series for $d \mu_{n}$,

$$
\rho_{k, n}=\frac{1}{\pi} \int_{-\pi}^{\pi} \cos k \tau d \mu_{n}(\tau)
$$


i.e., $d \mu_{n}(\tau) \sim \frac{1}{2}+\sum_{k=1}^{\infty} \rho_{k, n} \cos k \tau$.

Theorem 6.1 (Tureckiǐ, 1959, [21, Theorem 3.6]). Under the condition

$$
\lim _{n \rightarrow \infty} \frac{1-\rho_{k, n}}{1-\rho_{1, n}}=k^{2}, \quad k \in \mathcal{N}
$$

the sequence of positive convolution operators $\left\{L_{n}\right\}$ is saturated with order $\left(1-\rho_{1, n}\right)$ where $\rho_{k, n}$ 's are the real Fourier coefficients, and the saturation class is

$$
\begin{aligned}
S\left(L_{n}\right) & =\left\{f: \sum_{k=1}^{\infty} k^{2} A_{k}(f, x) \in L_{\infty}\right\} \\
& =\left\{f: f^{\prime} \in \operatorname{Lip} 1\right\} .
\end{aligned}
$$

In 1959, Komleva proved a condition equivalent to 6.4 is

$$
\int_{-\pi}^{\pi} \sin ^{4} \frac{t}{2} d \mu_{n}=o\left(1-\rho_{1, n}\right)
$$

Natanson used this fact and demonstrated another version of Tureckilı's Theorem.

Theorem 6.2 ([21, p. 77]). The sequence of positive convolution operator $\left\{L_{n}\right\}$ is saturated with order $\left(1-\rho_{1, n}\right)$ provided 6.5 holds.

In [21, Lemma 3.1], it is shown that for each $p \geq q \geq 1$, as $n$ tends to $\infty$, we have

$$
\int_{-\pi}^{\pi} \frac{\sin ^{2 p} \frac{n t}{2}}{\sin ^{2 q} \frac{t}{2}} d t \sim n^{2 q-1}
$$

Example 6.3. Let $\left\{\mathcal{J}_{n}\right\}$ be sequence of Jackson operator (3.5). We will calculate 
the saturation order of this sequence applying Theorem 6.2. First,

$$
\begin{aligned}
\int_{-\pi}^{\pi} \sin ^{4} \frac{t}{2} d \mu_{n} & =a_{n} \int_{-\pi}^{\pi} \sin ^{4} \frac{t}{2} \frac{\sin ^{4} \frac{n t}{2}}{\sin ^{4} \frac{t}{2}} d t \\
& =a_{n} \int_{-\pi}^{\pi} \sin ^{4} \frac{n t}{2} d t \\
& =\frac{9}{4}\left(4 n^{3}+2 n\right)^{-1} \\
& =\mathcal{O}\left(n^{-3}\right)
\end{aligned}
$$

On the other hand, we have

$$
\begin{aligned}
1-\rho_{1, n} & =\frac{1}{\pi} \int_{-\pi}^{\pi}(1-\cos t) d \mu_{n}(t) \\
& =\frac{2}{\pi} \int_{-\pi}^{\pi} \sin ^{2} \frac{t}{2} d \mu_{n}(t) \\
& =\frac{2}{\pi} a_{n} \int_{-\pi}^{\pi} \frac{\sin ^{4} \frac{n t}{2}}{\sin ^{2} \frac{t}{2}} d t \quad \text { by using 6.6. } \\
& \sim \frac{6}{\pi^{2}}\left(4 n^{3}+2 n\right)^{-1} n \\
& =n^{-2} .
\end{aligned}
$$

Thus, the sequence of Jackson operators is saturated with order $\left(n^{-2}\right)$ and the saturation class is

$$
S\left(\mathcal{J}_{n}\right):=\left\{f: f^{\prime} \in \operatorname{Lip} 1\right\}
$$

\subsubsection{Sunouchi-Watari Theorem}

Let $f$ be a $2 \pi$-periodic integrable function with the Fourier series

$$
\sum_{k=0}^{\infty} A_{k}(f, x)=\frac{a_{0}(f)}{2}+\sum_{k=1}^{\infty}\left(a_{k}(f) \cos k x+b_{k}(f) \sin k x\right)
$$


and let $P_{n}(x)$ denote the family of transforms of 6.7$)$ by a method of summation defined as follows:

$$
\begin{aligned}
P_{n}(x): & =\sum_{k=0}^{\infty} g_{k}(n) A_{k}(f, x) \\
& =\frac{a_{0}(f)}{2}+\sum_{k=1}^{\infty} g_{k}(n)\left(a_{k}(f) \cos k x+b_{k}(f) \sin k x\right),
\end{aligned}
$$

where $g_{k}(n)$ is summating function for $k=0,1,2, \ldots$ and $g_{0}(n)=1$.

Theorem 6.4 ([54, pp. 480-481]). Suppose there is a sequence of positive numbers $\{\varphi(n)\}$ converging to 0 and a positive function $\psi$ such that

$$
\lim _{n \rightarrow \infty} \frac{1-g_{k}(n)}{\varphi(n)}=c \psi(k)
$$

where $c$ is a positive costant. Then $\left\{P_{n}\right\}$ is saturated with order $(\varphi(n))$.

As a conclusion, if for $c, k, \rho>0$, we have

$$
\lim _{n \rightarrow \infty} n^{r}\left(1-g_{k}(n)\right)=c k^{\rho}
$$

then the saturation order is $\left(n^{-r}\right)$.

Example 6.5. In order to calculate the saturation order of the Fejér operator, we write

$$
\begin{aligned}
& \frac{1}{2 \pi(n+1)} \int_{-\pi}^{\pi} f(x+t)\left(\frac{\sin \frac{(n+1) t}{2}}{\sin \frac{t}{2}}\right)^{2} d t \\
& =\frac{1}{\pi} \int_{-\pi}^{\pi} f(x+t)\left\{\frac{1}{2}+\sum_{k=1}^{\infty}\left(1-\frac{k}{n+1}\right) \cos k t\right\} d t \\
& =\sum_{k=0}^{\infty}\left(1-\frac{k}{n+1}\right) A_{k}(x) \\
& =\sum_{k=0}^{\infty} g_{k}(n) A_{k}(x) .
\end{aligned}
$$


By using relation (6.10), we have

$$
\lim _{n \rightarrow \infty} n\left(1-g_{k}(n)\right)=\lim _{n \rightarrow \infty} n\left(\frac{k}{n+1}\right)=k .
$$

Thus, by the Sunouchi-Watari Theorem, the sequence of Fejér operators is saturated with order $\left(n^{-1}\right)$.

\subsection{Saturation in $C[a, b]$}

Let $[c, d] \subseteq[a, b]$. A sequence of operators $\left\{L_{n}\right\}$ from $C[a, b]$ into $C[c, d]$ is said to be saturated on $[c, d]$ if

1. there is a sequence of functions $\left\{\varphi_{n}(x)\right\}$ which are positive on $(c, d)$ and tend to 0 uniformly on $[c, d]$, and a class of functions $T\left(L_{n}\right)$ such that

$$
\lim _{n \rightarrow \infty}\left\|\left(\varphi_{n}(x)\right)^{-1}\left(f(x)-L_{n}(f, x)\right)\right\|=0
$$

if and only if

$$
f \in T\left(L_{n}\right)
$$

2. there is a function $f_{0} \in C[a, b], f_{0} \notin T\left(L_{n}\right)$ for which

$$
\lim _{n \rightarrow \infty}\left\|\left(\varphi_{n}(x)\right)^{-1}\left(f_{0}(x)-L_{n}\left(f_{0}, x\right)\right)\right\|=\mathcal{O}(1)
$$

Saturation order and trivial class of operators $L: C[a, b] \rightarrow C[a, b]$ is obtained in Theorem 6.6.

Theorem 6.6 ([21, Theorem 5.2]). Let $\left\{L_{n}\right\}$ be a sequence of positive operators from 
$C[a, b]$ to $C[a, b]$, and define

$$
\mu_{n}(x):=\frac{1}{2} L_{n}\left((t-x)^{2} ; x\right)
$$

If $\mu_{n}(x)>0$ for $x \in(a, b)$ and for $i=0,1$ we have

$$
\left\|\frac{x^{i}-L_{n}\left(t^{i} ; x\right)}{\mu_{n}(x)}\right\|=\mathrm{o}(1),
$$

then $\left\{L_{n}\right\}$ is saturated with order $\left(\mu_{n}(x)\right)$ and trivial class

$$
T\left(L_{n}\right)=\{l: l \text { is linear on }[a, b]\} .
$$

As an example, to find the saturation order for Bernstein operator s, we need to calculate $\mu_{n}(x)$.

$$
\mu_{n}(x)=\frac{1}{2} B_{n}\left((t-x)^{2} ; x\right)=\frac{x(1-x)}{2 n} .
$$

Thus, $\mu_{n}(x)>0$ for all $x \in(0,1)$. On the other hand, since $B_{n}\left(t^{i} ; x\right)=x^{i}$ for $i=0,1$, the condition (6.11) is satisfies. Therefore, the sequence of Bernstein operator is saturated with order $\left(\frac{x(1-x)}{2 n}\right)$ and trivial class

$$
T\left(B_{n}\right)=\{l: l \text { is linear on }[0,1]\} .
$$

As we can see, the saturation order in $C[a, b]$ could be a sequence of functions, i.e., $\left(\varphi_{n}(x)\right)$ while the saturation order in $C_{2 \pi}$ is a sequence of numbers, i.e., $(\varphi(n))$, since based on the position of $x$ in $[a, b]$ the degree of approximation of function will be changed. For instance, the Bernstein operator approximates $f$ near the endpoints of the interval better than in the middle. Also, Lorentz [38, Theorem 1.6.1] has proved that $B_{n}(f ; x)-f(x)=\mathcal{O}\left(\frac{1}{n}\right)$ if and only if $f^{\prime} \in$ Lip1. By applying Voronovskaja's result, this estimate could not be improve by increasing smoothness of function $f$ 
and the situation $B_{n}(f ; x)-f(x)=o\left(\frac{1}{n}\right)$ happens just for linear functions. 


\section{The Iteration of Positive Linear}

\section{Operators}

In 1967, Kelisky and Rivlin 34 proposed the iteration of the Bernstein operator, for all $f \in C[0,1]$ and $x \in[0,1]$,

$$
B_{n}^{k}(f ; x):=B_{n}\left(B_{n}^{k-1}(f ; x)\right), \quad k>1,
$$

and proved the convergence of the sequence $\left\{B_{n}^{k}\right\}_{k \geq 1}$ for fixed $n$ :

$$
\lim _{k \rightarrow \infty} B_{n}^{k}(f ; x)=f(0)+(f(1)-f(0)) x
$$

Karlin and Ziegler in [33, pp. 337-338] used iteration to determine the structure of the operators that commute with Bernstein operator, i.e., $U B_{n}=B_{n} U$. Also, they used the technique of iteration in order to characterize the class of functions $f$ such that for all $n, L(f) \geq f[33$, pp. 334-337].

The next example illustrates the Kelisky and Rivlin result. Let $f(x)=2+x^{4}$.

\footnotetext{
${ }^{1}$ Theodore J. Rivlin (1926-2006) received his Ph.D. from Harvard University in 1953 under the supervision of Joseph Walsh. He was an expert in approximation theory and computational mathematics. http://www.math.technion.ac.il/hat/people/rivlin.html
} 


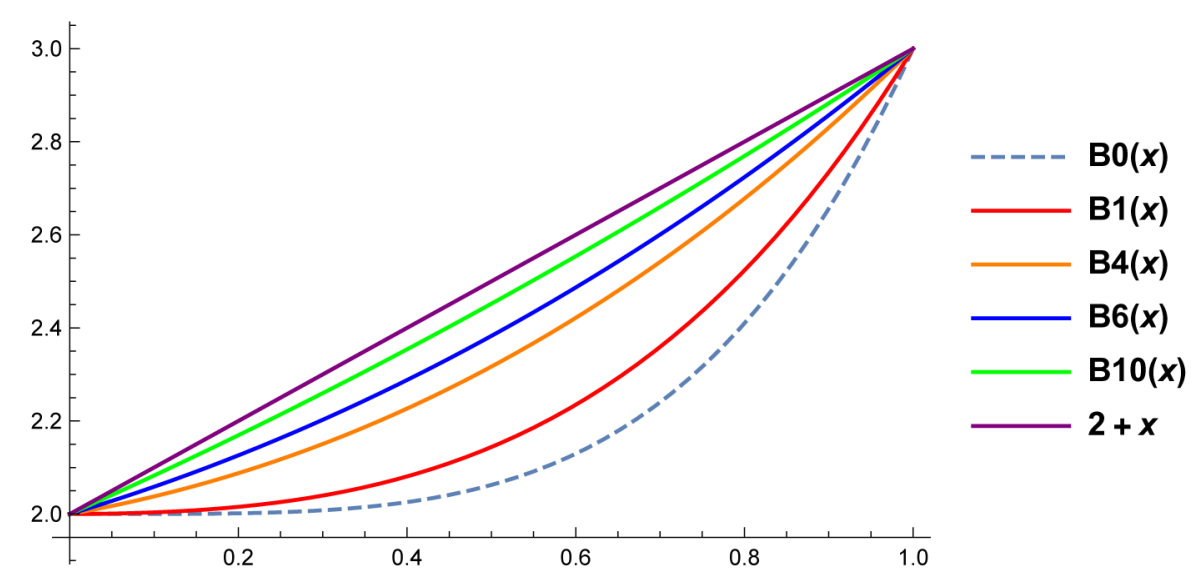

Figure 7.1: Iterates of the Bernstein operator of order $n=5$.

Our purpose is analyzing the iteration of the Bernstein operator for this function.

Example 7.1. For $k>1$, let $B_{n}^{k}(f ; x)=B_{n}\left(B_{n}^{k-1}(f) ; x\right)$. Then we have

$$
\lim _{k \rightarrow \infty} B_{n}^{k}(f ; x)=2+x
$$

Let $n$ be fixed and equal to 5. So we have

$$
\begin{aligned}
& B_{5}^{1}(f ; x)=2+0.008 x+0.224 x^{2}+0.576 x^{3}+0.192 x^{4} \\
& B_{5}^{4}(f ; x)=2+0.31983 x+0.575361 x^{2}+0.10345 x^{3}+0.00135895 x^{4} \\
& B_{5}^{6}(f ; x)=2+0.54312 x+0.432469 x^{2}+0.024361 x^{3}+0.0000500965 x^{4} \\
& B_{5}^{10}(f ; x)=2+0.808506 x+0.190196 x^{2}+0.00129837 x^{3}+6.80789 \times 10^{-8} x^{4} . \\
& B_{5}^{20}(f ; x)=2+0.958875 x+0.0411155 x^{2}+9.60935 \times 10^{-6} x^{3}+1.87018 \times 10^{-12} x^{4}
\end{aligned}
$$

In Figure 7.1, iterates of the Bernstein operator for $k=0,1,4,6,10$ are shown. It can be seen that as $k$ increases, the iterates of the Bernstein operator converge to $2+x$. We use Mathematica for all of calculation here.

In general, let $n, m \in \mathbb{N}$ and let $L_{n}: C[0,1] \rightarrow C[0,1]$ be a positive linear operator. We define the iteration of $L_{n}$ as

$$
\begin{aligned}
& L_{n}^{1}:=L_{n}, \\
& L_{n}^{m+1}:=L_{n} \circ L_{n}^{m} .
\end{aligned}
$$


Definition 7.2 (Weakly Picard operator, [48, p. 260]). Let $(X, d)$ be a metric space. The operator $L: X \rightarrow X$ is called a weakly Picard operator if for all $x \in X$, the sequence $\left\{L^{n}(x)\right\}_{n \geq 1}$ converges and the limit, which may depend on $x$, is a fixed point of $L$.

If $L$ is a weakly Picard operator, then define $L^{\infty}$ as follows

$$
L^{\infty}:=\lim _{n \rightarrow \infty} L^{n}
$$

Rus restated the Kelisky-Rivlin Theorem by stating that the Bernstein operator is a weakly Picard operator and

$$
B_{n}^{\infty}(f ; x)=f(0)+(f(1)-f(0)) x .
$$

Now, we investigate $\lim _{m \rightarrow \infty} L^{m}(f ; x)$ for the positive linear operator $L$, where

$$
L: C[0,1] \rightarrow C[0,1]
$$

There are several methods to study the behaviour of the iteration of operators such as the contraction principle method and the quantitative method.

Using the contraction principle, in 2003, Agratini and Rus proved the following result for the sequence of discrete interpolation positive linear operators $\left\{L_{n}\right\}$ defined on $C[0,1]$.

Theorem 7.3 ([3, Theorem 4]). Let $\left\{L_{n}\right\}$ be the sequence of discrete interpolation positive linear operators

$$
L_{n}: C[0,1] \rightarrow C[0,1]
$$


defined for all $f \in C[0,1]$ and $0 \leq x \leq 1$ by

$$
L_{n}(f, x)=\sum_{k=1}^{n} f\left(x_{n, k}\right) \varphi_{n, k}(x),
$$

where $\varphi_{n, k}(x) \in C[0,1]$ and $0=x_{n, 0}<x_{n, 1}<\cdots<x_{n, n}=1$, and the following conditions are satisfied

$$
\begin{gathered}
\varphi_{n, k}(x) \geq 0, \\
\varphi_{n, 0}(0)=\varphi_{n, n}(1)=1, \\
\sum_{k=0}^{n} \varphi_{n, k}(x)=1, \\
\sum_{k=0}^{n} x_{n, k} \varphi_{n, k}(x)=x . \\
\text { If } u_{n}:=\min _{x \in[0,1]}\left(\varphi_{n, 0}(x)+\varphi_{n, n}(x)\right)>0, \text { then } \\
\lim _{m \rightarrow \infty} L_{n}^{m}(f ; x)=(1-x) f(0)+x f(1) .
\end{gathered}
$$

As an example, the Bernstein operator satisfies the conditions of Theorem 7.3 and this yields the above mentioned Kelisky and Rivlin result.

Theorem 7.4 ([26, Theorem 1]). Let $L: C[0,1] \rightarrow C[0,1]$ be a positive linear operator preserving the affine functions. If there exist $\beta \geq 1$ and $\alpha>0$ such that

$$
L\left(t^{2} ; x\right)-x^{2} \geq \alpha\left(x-x^{2}\right)^{\beta}
$$

then

$$
\lim _{m \rightarrow \infty} L^{m}(f ; x)=(1-x) f(0)+x f(1)
$$

uniformly on $[0,1]$, for all $f \in C[0,1]$.

It is interesting to note that the Bernstein operator satisfies the conditions of 
Theorem 7.4:

$$
\begin{aligned}
B_{n}\left(t^{2} ; x\right)-x^{2} & =x^{2}-\frac{x^{2}}{n}+\frac{x}{n}-x^{2} \\
& =\frac{1}{n}\left(x-x^{2}\right),
\end{aligned}
$$

i.e., we can set $\alpha=\frac{1}{n}$ and $\beta=1$. Hence, we get the result of Kelisky and Rivlin as a consequence again.

In 2017, Birou showed the result of Agratini and Rus for the general positive linear operators $L_{n}$ defined on $C[0,1]$ which preserve linear functions.

Theorem 7.5 ([8, Theorem 2.1]). Let $L_{n}: C[0,1] \rightarrow C[0,1]$ be a sequence of positive linear operators satisfying

1. $L_{n}(1 ; x)=1$,

2. $L_{n}(t ; x)=x$,

3. $L_{n}\left(t^{2} ; x\right) \geq \alpha_{n} x^{2}+\left(1-\alpha_{n}\right) x$ with $0<\alpha_{n}<1$ and $0 \leq x \leq 1$.

Then, for all $f \in C[0,1]$, we have

$$
\lim _{m \rightarrow \infty} L_{n}^{m}(f ; x)=(1-x) f(0)+x f(1)
$$

Let $f \in C[0,1]$ and the operator $L_{n}$ satisfy the condition of Theorem 7.5 . Set $\delta=\sqrt{\alpha_{n}^{m} x(1-x)}>0$ and define

$$
B_{1} f(x):=(1-x) f(0)+x f(1) .
$$

By applying [47, Theorem 3.1], we have

$$
\begin{aligned}
\left|B_{1} f(x)-f(x)\right| & \leq|f(x)|\left|B_{1} e_{0}(x)-1\right|+\left|B_{1}\left(e_{1}-x e_{0}\right)(x)\right| \delta^{-1} \omega_{1}(f, \delta) \\
& +\left(B_{1} e_{0}+\frac{\delta^{-2}}{2} B_{1}\left(e_{1}-x e_{0}\right)^{2}(x)\right) \omega_{2}(f, \delta) .
\end{aligned}
$$


Since, $B_{1} e_{0}(x)=1$ and $B_{1} e_{1}(x)=x$, we get, for $x \in[0,1]$,

$$
\left|B_{1} f(x)-f(x)\right| \leq\left(B_{1} e_{0}(x)+\frac{\delta^{-2}}{2} B_{1}\left(e_{1}-x e_{0}\right)^{2}(x)\right) \omega_{2}(f, \delta) .
$$

This implies

$$
\left|B_{1} f-f\right| \leq\left(e_{0}+\frac{\delta^{-2}}{2}\left(e_{1}-e_{2}\right)\right) \omega_{2}(f, \delta) .
$$

Preserving linear functions by $L_{n}$, we obtain

$$
\left|B_{1} f-L_{n}^{m} f\right| \leq\left(e_{0}+\frac{\delta^{-2}}{2}\left(e_{1}-L_{n}^{m} e_{2}\right)\right) \omega_{2}(f, \delta)
$$

On the other hand,

$$
\begin{aligned}
e_{1}-L_{n}^{m} e_{2} & \leq e_{1}-\left(\alpha_{n}^{m} e_{2}+\left(1-\alpha_{n}^{m}\right) e_{1}\right) \\
& =\alpha_{n}^{m}\left(e_{1}-e_{2}\right) \\
& =\delta^{2} .
\end{aligned}
$$

Therefore, Birou obtained an estimate for the iterates of positive linear operators which satisfy conditions of Theorem 7.5 .

Theorem 7.6 ([8, Theorem 2.2]). Let $m, n \in \mathbb{N}$ and assume that $L_{n}$ and $f$ satisfy the conditions of Theorem 7.5. The following estimation holds:

$$
\left|L_{n}^{m}(f ; x)-((1-x) f(0)+x f(1))\right| \leq \frac{3}{2} \omega_{2}\left(f ; \sqrt{\alpha_{n}^{m} x(1-x)}\right)
$$

Applying this theorem to Bernstein operator s and setting $\alpha_{n}=1-1 / n$ we get the estimate

$$
\left|B_{n}^{m}(f ; x)-((1-x) f(0)+x f(1))\right| \leq \frac{3}{2} \omega_{2}\left(f ; \sqrt{\left(1-\frac{1}{n}\right)^{m} x(1-x)}\right) .
$$

Also, the genuine-Bernstein-Durrmeyer operator satisfies conditions of Theorems 7.5 
and 7.6, with $\alpha_{n}=1-2 /(n+1)$. This implies

$$
\left|U_{n}^{m}(f ; x)-((1-x) f(0)+x f(1))\right| \leq \frac{3}{2} \omega_{2}\left(f ; \sqrt{\left(1-\frac{2}{n+1}\right)^{m} x(1-x)}\right)
$$

Note that Theorem 7.5 can be used for the summation type operators which preserve linear functions, but it fails for operators like King's operator which preserve quadratics. Mahmudov considered discrete positive linear operators defined on $C[0,1]$ which preserve constant functions as well as quadratics. The following Theorem 7.7 is an analogue of Theorem 7.5

Theorem 7.7 ([40, Theorem 3]). Let $\left\{L_{n}\right\}$ be a sequence of discrete interpolation positive linear operators defined for all $f \in C[0,1]$ and $0 \leq x \leq 1$ by

$$
L_{n}(f, x)=\sum_{k=1}^{n} f\left(x_{n, k}\right) \varphi_{n, k}(x)
$$

where $\varphi_{n, k}(x) \in C[0,1]$ and $0=x_{n, 0}<x_{n, 1}<\cdots<x_{n, n}=1$, and the following conditions hold

$$
\begin{aligned}
& \varphi_{n, k}(x) \geq 0, \\
& \varphi_{n, 0}(0)=\varphi_{n, n}(1)=1, \\
& \sum_{k=0}^{n} \varphi_{n, k}(x)=1, \\
& \sum_{k=0}^{n} x_{n, k}^{2} \varphi_{n, k}(x)=x^{2} .
\end{aligned}
$$

If $u_{n}:=\min _{x \in[0,1]}\left(\varphi_{n, 0}(x)+\varphi_{n, n}(x)\right)>0$, then, for all $f \in C[0,1]$ and $x \in[0,1]$, we have

$$
\left|L_{n}^{m}(f ; x)-\left(\left(1-x^{2}\right) f(0)+x^{2} f(1)\right)\right| \leq 3 \omega_{2}(f ; \hat{\delta})+2 \omega_{1}(f ; \hat{\delta})+\frac{3}{2} \omega_{2}(f ; \sqrt{\hat{\delta}}),
$$

where $\hat{\delta}=\frac{1}{4}\left(1-u_{n}\right)^{m-1}\left(1-\varphi_{n, 0}(x)-\varphi_{n, n}(x)\right)$.

We now consider iteration of the King operator and obtain a result of Gonska 


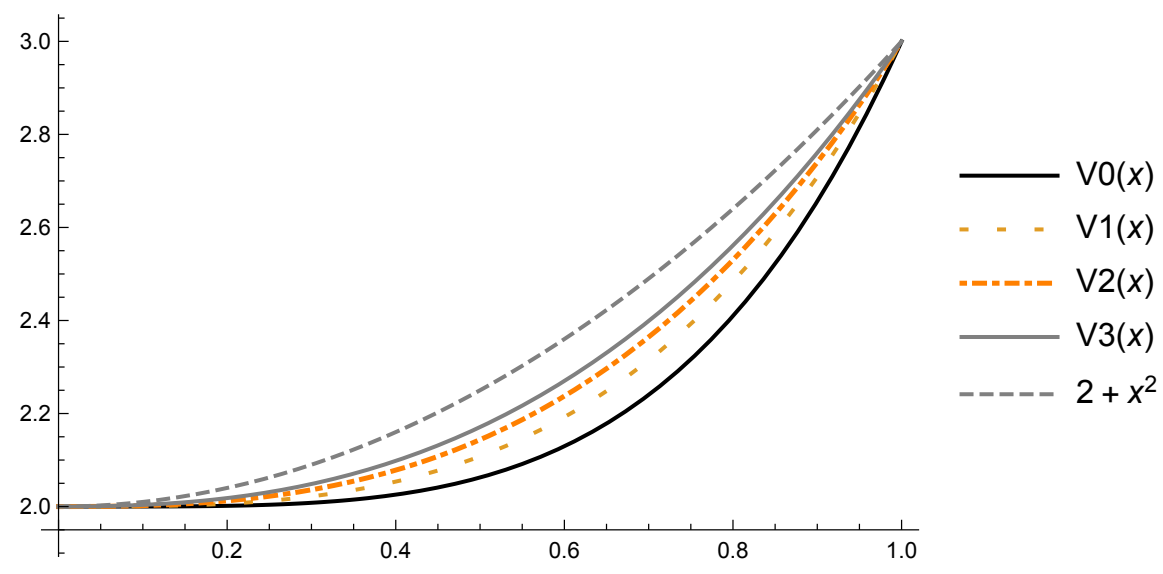

Figure 7.2: Iterates of the King operator for $f(x)=2+x^{4}$ when $n=5$.

and Pitul [27, Theorem 3.2] using a different method.

First, we check that the King operator satisfies conditions of Theorem 7.7 , Let

$$
\varphi_{n, k}(x)=\left(\begin{array}{l}
n \\
k
\end{array}\right)\left(r_{n}(x)\right)^{k}\left(1-r_{n}(x)\right)^{n-k}
$$

where $r_{n}(x)$ is defined in 3.12 . It is easy to verify that $\varphi_{n, k}(x) \geq 0$. On the other hand, for all $n>1, r_{n}(0)=0$ and $r_{n}(1)=1$ imply that

$$
\begin{aligned}
& \varphi_{n, 0}(0)=\left(\begin{array}{l}
n \\
0
\end{array}\right)\left(1-r_{n}(0)\right)^{n}=1, \\
& \varphi_{n, n}(1)=\left(\begin{array}{l}
n \\
n
\end{array}\right) r_{n}(1)^{n}=1 .
\end{aligned}
$$

Since the King operator preserves constants and $x^{2}$,

$$
\begin{aligned}
& \sum_{k=0}^{n} \varphi_{n, k}(x)=1, \\
& \sum_{k=0}^{n} x_{n, k}^{2} \varphi_{n, k}(x)=x^{2} .
\end{aligned}
$$


Since $0 \leq r_{n}(x) \leq 1$,

$$
\begin{aligned}
u_{n} & =\min _{x \in[0,1]}\left(\varphi_{n, 0}(x)+\varphi_{n, n}(x)\right) \\
& =\min _{x \in[0,1]}\left(\left(1-r_{n}(x)\right)^{n}+\left(r_{n}(x)\right)^{n}\right)>0 .
\end{aligned}
$$

Hence, for all $f \in C[0,1]$, we have

$$
\lim _{k \rightarrow \infty} V_{n}^{k}(f ; x)=\left(1-x^{2}\right) f(0)+x^{2} f(1) .
$$

Figure 7.2 illustrates the iteration of the King operator for $f(x)=2+x^{4}$ when $n=5$.

Weakly Picard operator has roles in the field of differential equations such as investigating existence solution for integral equation [57, Theorem 3.1]. Another application of iteration would be increasing the order of approximation of the Bernstein operator. Micchelli [43] and four years later, Mastroianni and Occorsio [41] considered the following iteration

$$
\oplus^{M} B_{n}:=I-\left(I-B_{n}\right)^{M},
$$

where $B_{n}$ is the Bernstein operator and $M \geq 1$. For any $f \in C[0,1]$, Gonska and Zhou showed the following theorem.

Theorem 7.8. [28, Theorem 1]

Let

$$
\omega_{\varphi}^{2 M}(f, t):=\sup _{h \in(0, t]}\left\|\Delta_{h \varphi}^{2 M} f\right\|
$$

where, $\varphi(x)=\sqrt{x(1-x)}$ and for $x \pm \operatorname{Mh} \varphi(x) \in[-1,1]$ define

$$
\Delta_{h \varphi}^{2 M} f(x):=\sum_{k=0}^{2 M}(-1)^{k}\left(\begin{array}{c}
2 M \\
k
\end{array}\right) f(x+(M-k) h \varphi(x)),
$$


otherwise, it is zero. For $M \geq 1$ fixed and any $f \in C[0,1]$, we have

$$
\left\|f-\oplus^{M} B_{n} f\right\| \leq C\left(\omega_{\varphi}^{2 M}\left(f, \frac{1}{\sqrt{n}}\right)+\|f\| n^{-M}\right)
$$

where $C$ is a constant independent of $f$ and $n$.

For instance, from (7.8) for $M=1$, we obtain

$$
\left\|f-B_{n}(f)\right\| \leq C\left(\omega_{\varphi}^{2}\left(f, \frac{1}{\sqrt{n}}\right)+\|f\| n^{-1}\right) .
$$

However, [56, p. 995], it was shown that

$$
\left\|f-B_{n}(f)\right\| \approx \omega_{\varphi}^{2}\left(f, \frac{1}{\sqrt{n}}\right)
$$

where $\approx$ means that the ratio of $\left\|f-B_{n}(f)\right\|$ and $\omega_{\varphi}^{2}\left(f, \frac{1}{\sqrt{n}}\right)$ is between two positive constants and it is independent of $n$ and $f$. 


\section{Bibliography}

[1] Abel, U., And Ivan, M. Some identities for the operator of Bleimann, Butzer and Hahn involving divided differences. Calcolo 36, 3 (1999), 143-160.

[2] Adell, J. A., and de la Cal, J. Bernstein-Durrmeyer operators. Comput. Math. Appl. 30, 3-6 (1995), 1-14. Concrete analysis.

[3] Agratini, O., And Rus, I. A. Iterates of a class of discrete linear operators via contraction principle. Comment. Math. Univ. Carolin. 44, 3 (2003), 555-563.

[4] Albrycht, J., And Radecki, J. On a generalization of the theorem of Voronovskaya. Zeszyty Nauk. Uniw. Mickiewicza No. 25 (1960), 3-7.

[5] Altomare, F. Korovkin-type theorems and approximation by positive linear operators. Surv. Approx. Theory 5 (2010), 92-164.

[6] Attalıenti, A., And Raşa, I. Total positivity: an application to positive linear operators and to their limiting semigroups. Rev. Anal. Numér. Théor. Approx. 36, 1 (2007), 51-66 (2008).

[7] Baskakov, V. A. An instance of a sequence of linear positive operators in the space of continuous functions. Dokl. Akad. Nauk SSSR (N.S.) 113 (1957), 249-251.

[8] Birou, M. M. New rates of convergence for the iterates of some positive linear operators. Mediterr. J. Math. 14, 3 (2017), Art. 129, 12. 
[9] Bleimann, G., Butzer, P. L., And Hahn, L. A Bernšteŭn-type operator approximating continuous functions on the semi-axis. Nederl. Akad. Wetensch. Indag. Math. 42, 3 (1980), 255-262.

[10] Bohman, H. On approximation of continuous and of analytic functions. Ark. Mat. 2 (1952), 43-56.

[11] Bohman, H. On approximation of continuous and of analytic functions. Ark. Mat. 2 (1952), 43-56.

[12] Boyanov, B. D., And Veselinov, V. M. A note on the approximation of functions in an infinite interval by linear positive operators. Bull. Math. Soc. Sci. Math. R. S. Roumanie (N.S.) 14(62) (1970), 9-13 (1971).

[13] Butzer, P. L., And Nessel, R. J. Fourier analysis and approximation. Academic Press, New York-London, 1971. Volume 1: One-dimensional theory, Pure and Applied Mathematics, Vol. 40.

[14] Cárdenas-Morales, D., Garrancho, P., and Raşa, I. Asymptotic formulae via a Korovkin-type result. Abstr. Appl. Anal. (2012), Art. ID 217464, 12.

[15] Cheney, E. W., And Sharma, A. Bernstein power series. Canad. J. Math. 16 (1964), 241-252.

[16] Chlodovsky, I. Sur le développement des fonctions définies dans un intervalle infini en séries de polynomes de M. S. Bernstein. Compositio Math. 4 (1937), 380-393.

[17] de la Cal, J., ANd CÁrcamo, J. An extremal property of Bernstein operators. J. Approx. Theory 146, 1 (2007), 87-90. 
[18] DE la CAL, J., AND CÁRCAMo, J. On the approximation of convex functions by Bernstein-type operators. J. Math. Anal. Appl. 334, 2 (2007), 1106-1115.

[19] DE LeEuW, K. On the degree of approximation by Bernstein polynomials. J. Analyse Math. 7 (1959), 89-104.

[20] Derriennic, M. M. Sur l'approximation de fonctions intégrables sur [0, 1] par des polynômes de Bernstein modifiés. J. Approx. Theory 31, 4 (1981), 325-343.

[21] DeVore, R. A. The approximation of continuous functions by positive linear operators. Lecture Notes in Mathematics, Vol. 293. Springer-Verlag, Berlin-New York, 1972.

[22] DeVore, R. A., And Lorentz, G. G. Constructive approximation, vol. 303 of Grundlehren der Mathematischen Wissenschaften [Fundamental Principles of Mathematical Sciences]. Springer-Verlag, Berlin, 1993.

[23] Ditzian, Z. Polynomial approximation and $\omega_{\phi}^{r}(f, t)$ twenty years later. Surv. Approx. Theory 3 (2007), 106-151.

[24] GAL, S. G. Approximation by complex Bernstein and convolution type operators, vol. 8 of Series on Concrete and Applicable Mathematics. World Scientific Publishing Co. Pte. Ltd., Hackensack, NJ, 2009.

[25] Gavrea, I., Gonska, H. H., And Kacsó, D. P. On the variationdiminishing property. Results Math. 33, 1-2 (1998), 96-105.

[26] Gavrea, I., And Ivan, M. On the iterates of positive linear operators preserving the affine functions. J. Math. Anal. Appl. 372, 2 (2010), 366-368.

[27] Gonska, H., And Piţul, P. Remarks on an article of J. P. King: "Positive linear operators which preserve $x^{2} "$ [Acta Math. Hungar. 99 (2003), no. 3, 203208; mr1973095]. Comment. Math. Univ. Carolin. 46, 4 (2005), 645-652. 
[28] Gonska, H. H., And Zhou, X. L. Approximation theorems for the iterated Boolean sums of Bernstein operators. J. Comput. Appl. Math. 53, 1 (1994), $21-31$.

[29] Goodman, T. N. T., Oruç, H., and Phillips, G. M. Convexity and generalized Bernstein polynomials. Proc. Edinburgh Math. Soc. (2) 42, 1 (1999), 179-190.

[30] Heping, W. Korovkin-type theorem and application. J. Approx. Theory 132, 2 (2005), 258-264.

[31] Karlin, S. Total positivity, absorption probabilities and applications. Trans. Amer. Math. Soc. 111 (1964), 33-107.

[32] Karlin, S. Total positivity. Vol. I. Stanford University Press, Stanford, Calif, 1968.

[33] Karlin, S., And Ziegler, Z. Iteration of positive approximation operators. J. Approximation Theory 3 (1970), 310-339.

[34] Kelisky, R. P., And Rivlin, T. J. Iterates of Bernstein polynomials. Pacific J. Math. 21 (1967), 511-520.

[35] King, J. P. Positive linear operators which preserve $x^{2}$. Acta Math. Hungar. 99, 3 (2003), 203-208.

[36] Konovkin, P. P. On convergence of linear positive operators in the space of continuous functions. Doklady Akad. Nauk SSSR (N.S.) 90 (1953), 961-964.

[37] Konovkin, P. P. Linear operators and approximation theory. Translated from the Russian ed. (1959). Russian Monographs and Texts on Advanced Mathematics and Physics, Vol. III. Gordon and Breach Publishers, Inc., New York; Hindustan Publishing Corp. (India), Delhi, 1960. 
[38] Lorentz, G. G. Bernstein polynomials. Mathematical Expositions, no. 8. University of Toronto Press, Toronto, 1953.

[39] Lupas, A., AND MÜller, M. W. Approximation properties of the $M_{n^{-}}$ operators. Aequationes Math. 5 (1970), 19-37.

[40] Mahmudov, N. I. Asymptotic properties of powers of linear positive operators which preserve $e_{2}$. Comput. Math. Appl. 62, 12 (2011), 4568-4575.

[41] Mastroianni, G., And Occorsio, M. R. A generalization of the Bernšteŭn operator. Rend. Accad. Sci. Fis. Mat. Napoli (4) 44 (1977), 151-169 (1978).

[42] MatsuokA, Y. On the approximation of functions by some singular integrals. Tôhoku Math. J. (2) 18 (1966), 13-43.

[43] Micchelli, C. A. The saturation class and iterates of the Bernstein polynomials. J. Approximation Theory 8 (1973), 1-18. Collection of articles dedicated to Isaac Jacob Schoenberg on his 70th birthday, I.

[44] Natanson, I. P. Konstruktive Funktionentheorie. Akademie-Verlag, Berlin, 1955.

[45] Pinkus, A. Totally positive matrices, vol. 181 of Cambridge Tracts in Mathematics. Cambridge University Press, Cambridge, 2010.

[46] Pólya, G., And Schoenberg, I. J. Remarks on de la Vallée Poussin means and convex conformal maps of the circle. Pacific J. Math. 8 (1958), 295-334.

[47] PăLtănea, R. Optimal estimates with moduli of continuity. Results Math. 32, 3-4 (1997), 318-331.

[48] Rus, I. A. Iterates of Bernstein operators, via contraction principle. J. Math. Anal. Appl. 292, 1 (2004), 259-261. 
[49] Schoenberg, I. J. On variation diminishing approximation methods. In On numerical approximation. Proceedings of a Symposium, Madison, April 21-23, 1958, Edited by R. E. Langer. Publication no. 1 of the Mathematics Research Center, U.S. Army, the University of Wisconsin. The University of Wisconsin Press, Madison, 1959, pp. 249-274.

[50] SCHuRER, F. On linear positive operators in approximation theory, vol. 1965 of Dissertation, Technological University of Delft. Uitgeverij Waltman, Delft, 1965.

[51] Shisha, O., And Mond, B. The degree of convergence of sequences of linear positive operators. Proc. Nat. Acad. Sci. U.S.A. 60 (1968), 1196-1200.

[52] Stancu, D. D. On the monotonicity of the sequence formed by the first order derivatives of the Bernstein polynomials. Math. Z. 98 (1967), 46-51.

[53] StečKin, S. B. On the order of the best approximations of continuous functions. Izvestiya Akad. Nauk SSSR. Ser. Mat. 15 (1951), 219-242.

[54] Sunouchi, G.-I., And Watari, C. On the determination of the class of saturation in the theory of approximation of functions. II. Tôhoku Math. J. (2) 11 (1959), 480-488.

[55] Suzuki, Y., And Watanabe, S. Some remarks on saturation problem in the local approximation. II. Tôhoku Math. J. (2) 21 (1969), 65-83.

[56] Tотік, V. Approximation by Bernstein polynomials. Amer. J. Math. 116, 4 (1994), 995-1018.

[57] Wang, J., Zhou, Y., And Medvě̌, M. Picard and weakly Picard operators technique for nonlinear differential equations in Banach spaces. J. Math. Anal. Appl. 389, 1 (2012), 261-274. 
[58] Xu, X.-W., Zeng, X.-M., and Goldman, R. Shape preserving properties of univariate Lototsky-Bernstein operators. J. Approx. Theory 224 (2017), 13-42. 


\section{Index}

Baskakov Operator, 25, 38, 57

Bernstein Operator, 1,4, 16, 17, 22, 23.

26, 30, 36, 42, 45, 46, 55, 61, 67,

$78,80,83,85,88$

Bernstein-Chlodovsky Operator, 16, 23.

43. 68

Bleimann-Butzer-Hahn Operator, 28, 54.

65

de la Vallée Poussin Operator, 18, 52, 60

Durrmeyer Operator, 26, 34, 35, 38, 40.

45. 63

Favard-Szász-Mirakjan Operator, 24, 38.

43, 56

Fejér Operator, 4, 19, 76

genuine-Bernstein-Durrmeyer Operator, 85

Jackson Operator, 4, 21, 51, 60, 75

Kantorovich Operator, 22, 38

King Operator, 28, 86, 88

Meyer-König-Zeller Operator, 5,68

Modified Durrmeyer Operator, 44, 45, 63 